\title{
Chemical Analyses of Soils, Soil Leaches, Rocks, and Stream Sediments from Guam and the Western United States and Sample Location Maps of Guam
}

By William R. Miller, Richard F. Sanzolone, Paul J. Lamothe, and Thomas L. Ziegler ${ }^{1}$

Open-File Report 02-399

\section{2}

U.S. Department of the Interior

U.S. Geological Survey

${ }^{1}$ U.S. Geological Survey, Denver, CO 80225 


\section{TABLE OF CONTENTS}

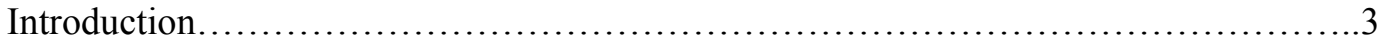

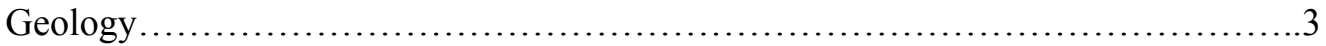

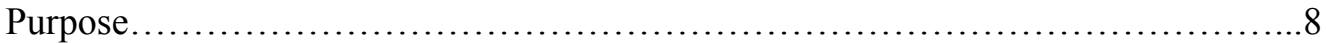

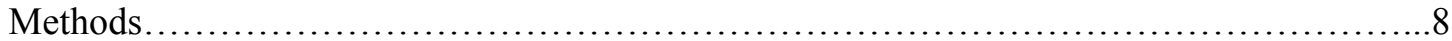

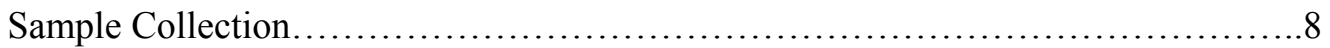

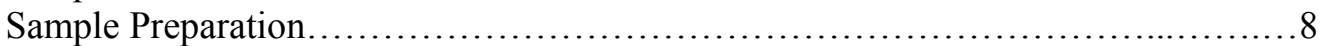

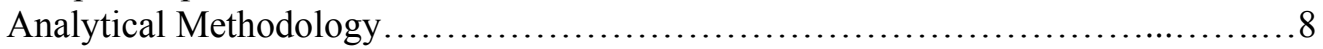

Total Elemental Composition............................................8

Soil $\mathrm{pH}$ Determination...............................................14

Water-Soluble Extraction.............................................14

Sequential Partial Dissolution Technique...............................14

Simulated Lung Fluid Dissolution....................................16

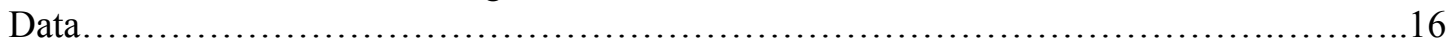

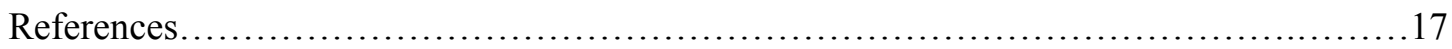

\section{Tables}

Table 1. Chemical analyses of soils from Guam................................... 19

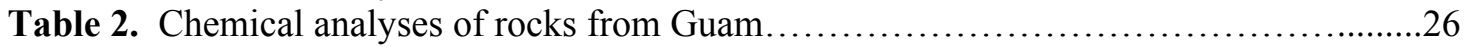

Table 3. Chemical analyses of streambed sediments from areas underlain by the Facpi Formation, Guam.................................................33

Table 4. Chemical analyses of soil samples from the western United States..................40

Table 5. Chemical analyses of rocks from the western United States.......................47

Table 6. Chemical analyses of water-soluble extractions of soils from Guam.................54

Table 7. Chemical analyses of water-soluble extractions of soils from the western United States....................................................... 57

Table 8. Chemical analyses of sequential extractions of soils from Guam and Colorado.......60

Table 9. Chemical analyses of simulated lung fluid dissolution of soils from Guam

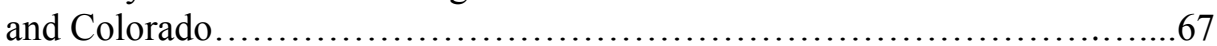

\section{Figures}

Figure 1. Location of the three southern villages in Guam with high incidences of

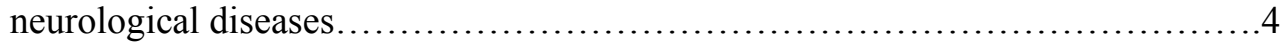

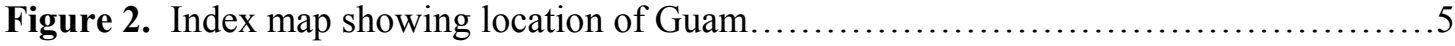

Figure 3. Generalized geologic map of Guam...........................................

Figure 4. Map showing locations of sample sites within the Facpi Formation in the vicinity of Umatac. ...

Figure 5. Map showing locations of sample sites within the Facpi Formation in the vicinity of Merizo.

Figure 6. Map showing locations of sample sites within the Bolanos Formation in the vicinity of Inarajan.

Figure 7. Map showing locations of sample sites within the Facpi Formation in the vicinity of Sella Bay and Cetti Bay.....

Figure 8. Map showing locations of sample sites within the Alutom Formation (except site S18 which is within the carbonate rocks) in the central part of Guam................ 13 


\section{Introduction}

A high incidence of neurodegenerative diseases, mainly dementia, parkinsonism, and amyotrophic lateral sclerosis, occurs on the island of Guam (Koerner, 1952, Kurland and others, 1954). High incidences of the diseases are concentrated along the southern coast of Guam, particularly the villages of Umatac, Merizo, and Inarajan (fig. 1). Volcanic rocks underlie the vicinities of the villages. The Northern part of Guam, with lower incidence of the diseases, consists of carbonate rocks. Epidemiological studies beginning in the early 1950's failed to show the cause to be genetic etiology (Plato and others, 1986, Zhang and others, 1990). In recent studies, the search for pathogenic mechanisms has shifted to environmental factors. Some of the studies investigated metal exposure, particularly aluminum and manganese, and deficiencies in calcium and magnesium (Garruto and others, 1984). Aluminum has been shown to have neurotoxic effects (MacDonald and Martin, 1988), and aluminum has been implicated in the pathogenesis of Alzheimer's disease and similar dementia by Perl and others (1982). Studies of soils developed on volcanic rocks on Guam and other islands by Crapper McLachlan and others (1989) found that soils on Guam averaged 42-fold higher yield of elutable aluminum than soils developed on volcanic rocks on Jamaica or Palau. They did not detect unusually high dietary aluminum or low dietary calcium but concluded that the soils and possibly the dusts of Guam might be a major source of aluminum entering the body of the inhabitants.

This study was conducted to investigate the geochemistry of the soils and rocks of the volcanic southern half of the island of Guam, particularly in the vicinity of three villages (Umatac, Merizo, and Inarajan) with high incidence of the diseases. In addition to total chemical analyses of the soils and rocks, leaches of soils were carried out. Because soluble aluminum in the soil was shown by Crapper McLachlan and others (1989) to be unusually high, distilled water leaches of the soils as well as partial sequential leaches, and leaches using simulated lung fluid were carried out.

In order to compare the results of the chemical data of rocks and soils from Guam to other rocks and soils elsewhere, samples of similar rocks and soils were collected in the western United States and similar analyses to those for the Guam samples carried out.

\section{Geology}

The island of Guam is located at the southern end of the Mariana fore-arc (fig. 2). The volcanic rocks which occur on the southern portion of Guam consist of, from oldest to youngest, late-middle Eocene Facpi Formation consisting of submarine boninite pillow flows and breccias, tuffaceous shale and sandstone, basaltic to andesitic dikes and minor limestone; the late Eocene to early Oligocene Alutom Formation consisting of tuffaceous shale and sandstone, volcanic breccia, conglomerate, and minor lava flows; and the Miocene Bolanos Formation of tuff breccia, tuffaceous sandstone, lenses of volcanic conglomerate, and minor basalt flows (Tracey and others, 1964; Reagan and Meijer, 1984; Siegrist and Randall, 1992). The Facpi Formation underlies the vicinity of the villages of Umatac and Merizo, and the Bolanas Formation underlies the vicinity of Inarajan (fig. 1). The northern portion of Guam consists of Tertiary to Quaternary carbonate rock (Tracey and others, 1964) (fig. 3). 


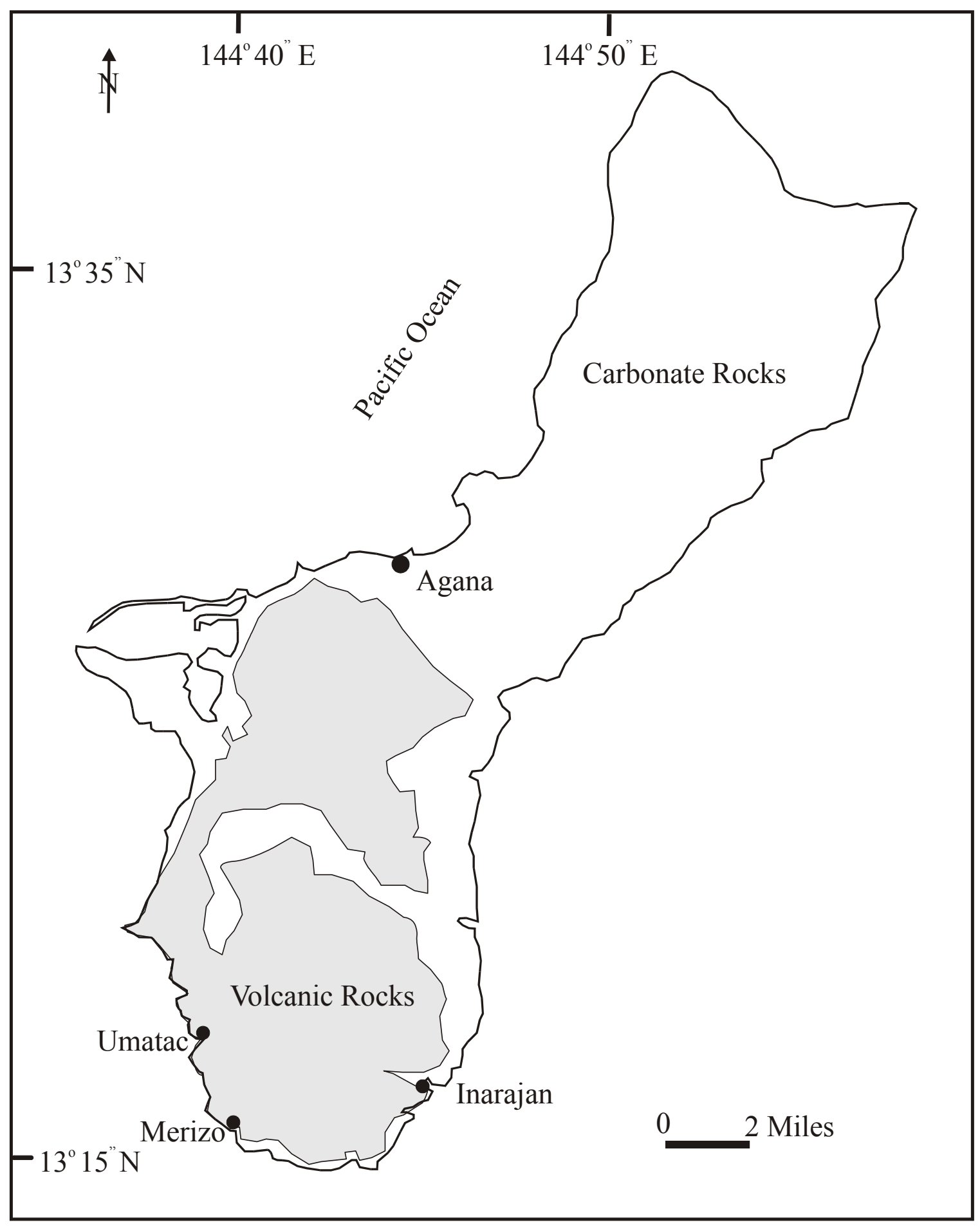

Figure 1. Location of the three southern villages with high incidences of neurological diseases, Guam 


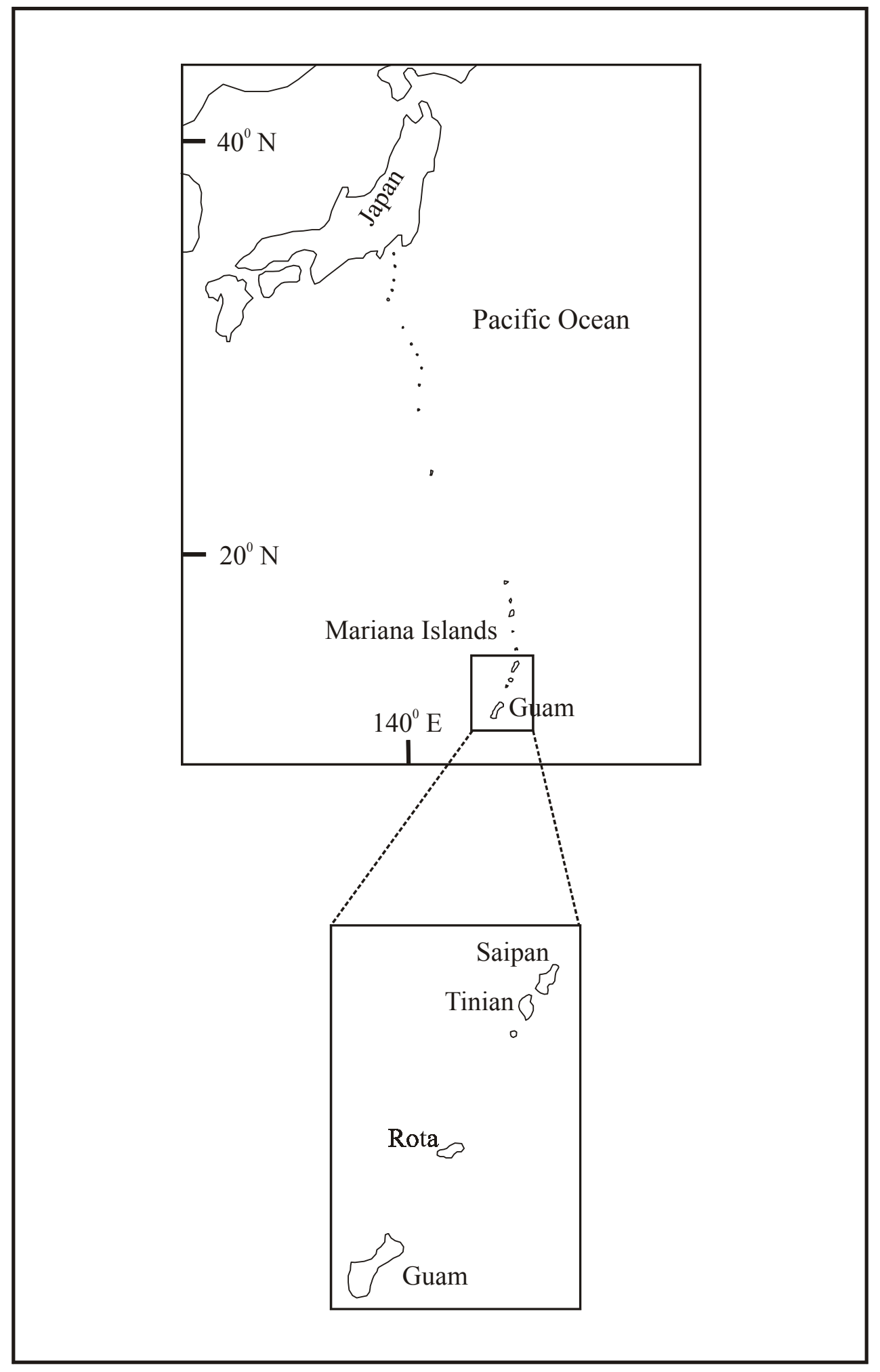

Figure 2. Index map showing location of Guam 


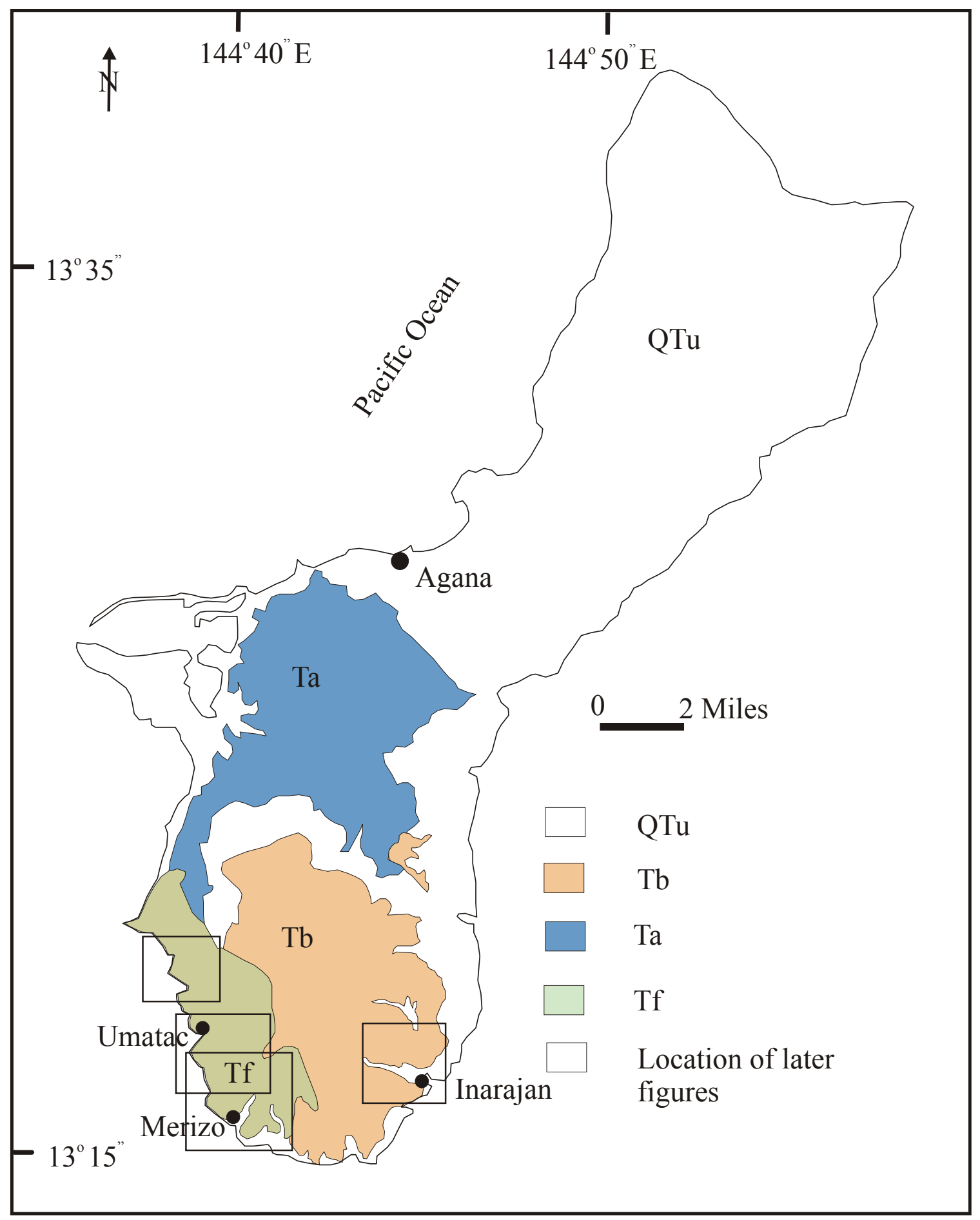

Figure 3. Generalized geologic map of Guam (modified from Tracey and others, 1964, and Siegrist and Randall, 1992). See next page for map explanation. 
Generalized Geologic Map of Guam

Explanation

\begin{tabular}{|c|c|c|l|}
\hline Age & Map symbol & $\begin{array}{c}\text { Formation or rock } \\
\text { type }\end{array}$ & \multicolumn{1}{|c|}{ Description } \\
\hline $\begin{array}{c}\text { Tertiary to } \\
\text { Quaternary }\end{array}$ & Qtu & Carbonate rocks & Carbonate rocks \\
\hline Miocene & Tb & Bolanos Formation & $\begin{array}{l}\text { Pillow basalt, tuffaceous shale } \\
\text { and shale, limestone, tuff } \\
\text { breccia, and volcanic } \\
\text { conglomerate. }\end{array}$ \\
\hline $\begin{array}{c}\text { Eocene and } \\
\text { Oligocene }\end{array}$ & Ta & Alutom Formation & $\begin{array}{l}\text { Tuffaceous shale and } \\
\text { sandstone, volcanic breccia, } \\
\text { conglomerate and minor } \\
\text { interbedded lava flows. }\end{array}$ \\
\hline Eocene & Tf & Facpi Volcanics & $\begin{array}{l}\text { Submarine boninite pillow } \\
\text { flows and breccias, tuffaceous } \\
\text { sediments, tholeiitic basalt } \\
\text { dikes, minor pelagic carbonate } \\
\text { sediment. }\end{array}$ \\
\hline
\end{tabular}


The volcanic rocks are weathered to an average depth of 50 feet (Carroll and Hathaway, 1963). Soils developed on the volcanic rocks average 20 to $40 \mathrm{~cm}$ in depth with thicker soils on valley floors and narrow coastal plain areas (Young, 1988). The main difference in soil thickness is due to topography. The general process in the soil formation is that glass, zeolites, plagioclase, and pyroxenes in rocks are weathered and transformed to smectite to kaolinite to gibbsite with increasing weathering intensity. The net effect is that silica is dissolved and removed and $\mathrm{Fe}_{2} \mathrm{O}_{3}$ and $\mathrm{Al}_{2} \mathrm{O}_{3}$ accumulate (Carroll and Hathaway, 1963).

\section{Purpose}

The purpose of this report is to present the complete chemical data of the soils, rocks, stream sediments, water leaches, sequential partial dissolution, and soil leach using simulated lung fluid of samples collected from Guam and the western United States. The data are presented without interpretation. A later report will interpret the data.

\section{Methods}

\section{Sample Collection}

Samples consisted of the collection of 25 soils, 15 rocks, and 4 streambed sediment samples in Guam in March 2001 (figs. 4-8). The soils were collected from the surface to a depth of about $10 \mathrm{~cm}$. Rocks were collected by compositing chips of rock from surface outcrops. The rocks were all weathered to some degree and do not represent original unweathered rock. Results of chemical analyses of unweathered rocks from Guam can be found in Reagan and Meijer (1984). Stream-bed sediments were collected by compositing material from several locations in the stream channel.

To allow comparisons of the chemical data from Guam to similar rocks and soils elsewhere, samples of 36 soils and 10 rocks were collected in the western United States. The western U.S. soils were poorly developed and contained significant weathered rock material. The samples were sieved through a $<2 \mathrm{~mm}$ stainless steel sieve during collection. Samples of rocks were collected by compositing chips from surface outcrops.

\section{Sample Preparation}

In the laboratory, soil and stream sediment samples were dried in a convection oven at ambient temperature and split using a Jones splitter. A split was then sieved to < 63 microns. Rock samples were put through a jaw crusher and then ground to less-than100 mesh ( $<150$ micron) using a ceramic plate grinder prior to analysis. Soil samples were sieved using a 63-micron stainless-steel sieve and the material passing through the sieve was used for all chemical analysis. The $<63$ micron size fraction makes up the majority of the soil sample material.

\section{Analytical Methodology}

Total Elemental Composition: The total element composition for rock, soil, and streambed sediment samples was determined for 56 elements by inductively coupled plasma - mass spectrometry (ICP-MS) after a multi-acid (hydrochloric, hydrofluoric, nitric, and perchloric) decomposition (Briggs and Meier, 1999). The elements 


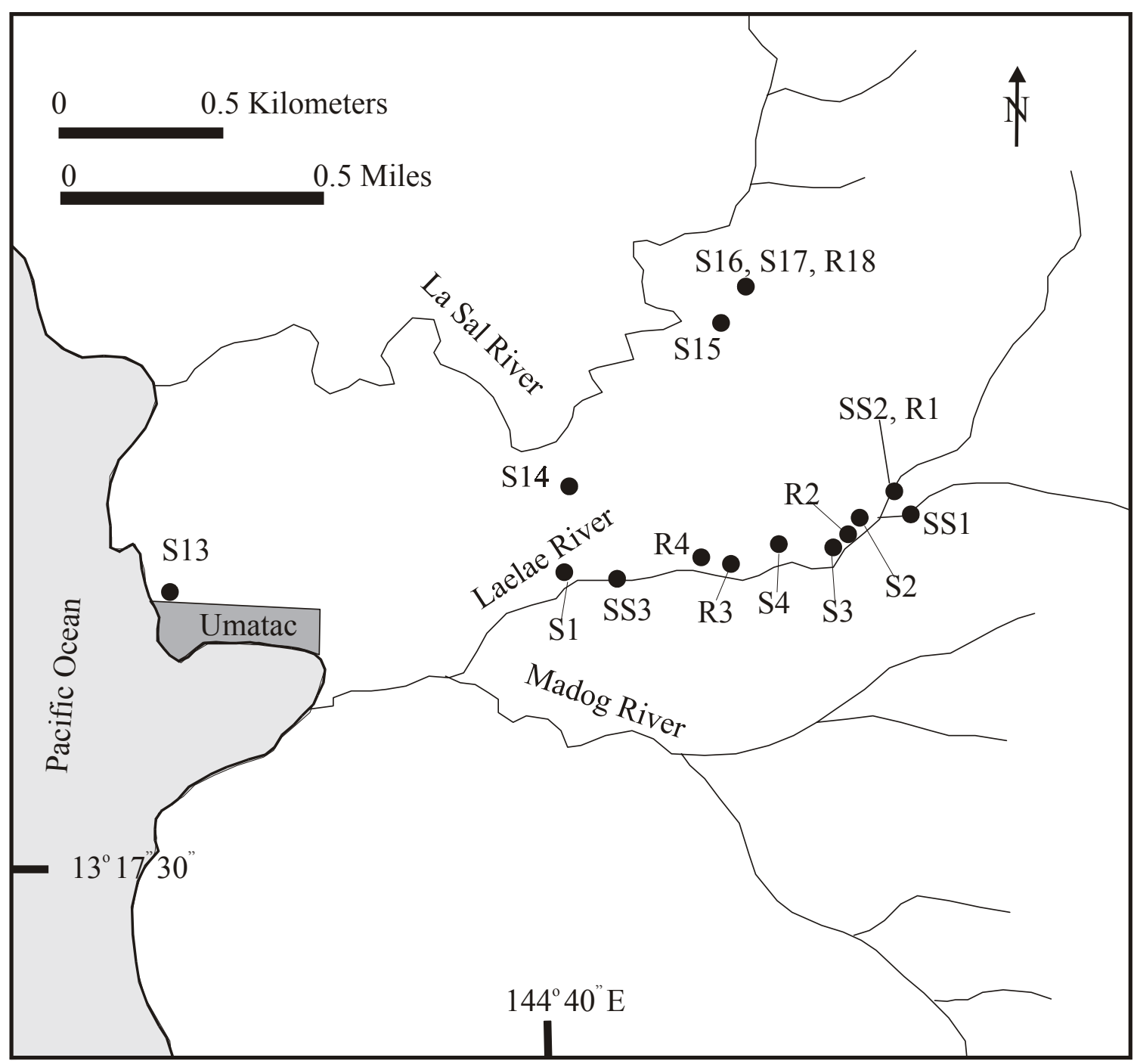

Figure 4. Map showing locations of sample sites within the Facpi Formation in the vicinity of Umatac. Sites prefixed with "S" are soils, "R" are rocks, and "SS" are stream sediments. 


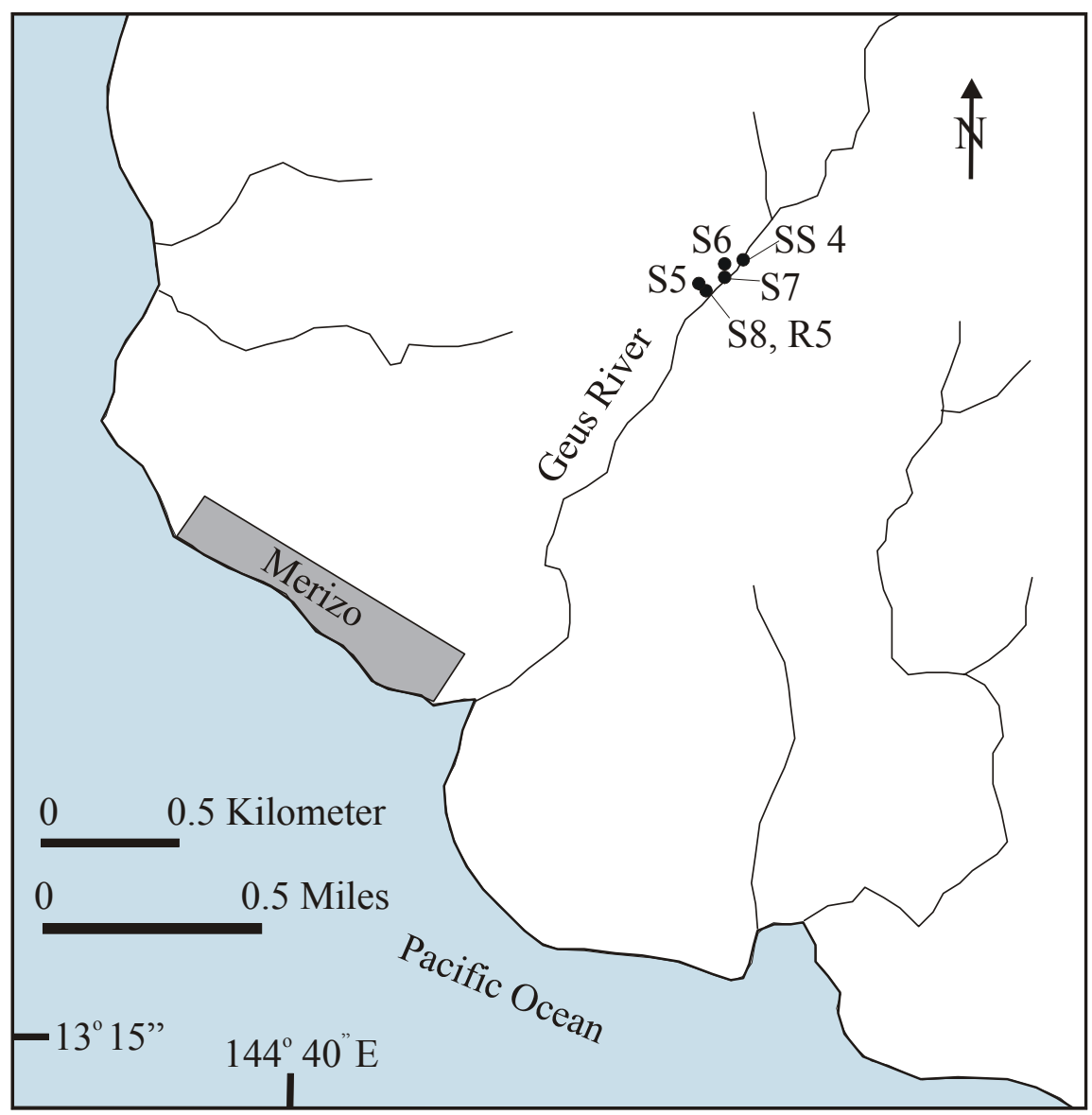

Figure 5. Map showing locations of sample sites within the Facpi Formation in the vicinity of Merizo. Sites prefixed with "S" are soils, "R" are rocks, and "SS" are stream sediments. 


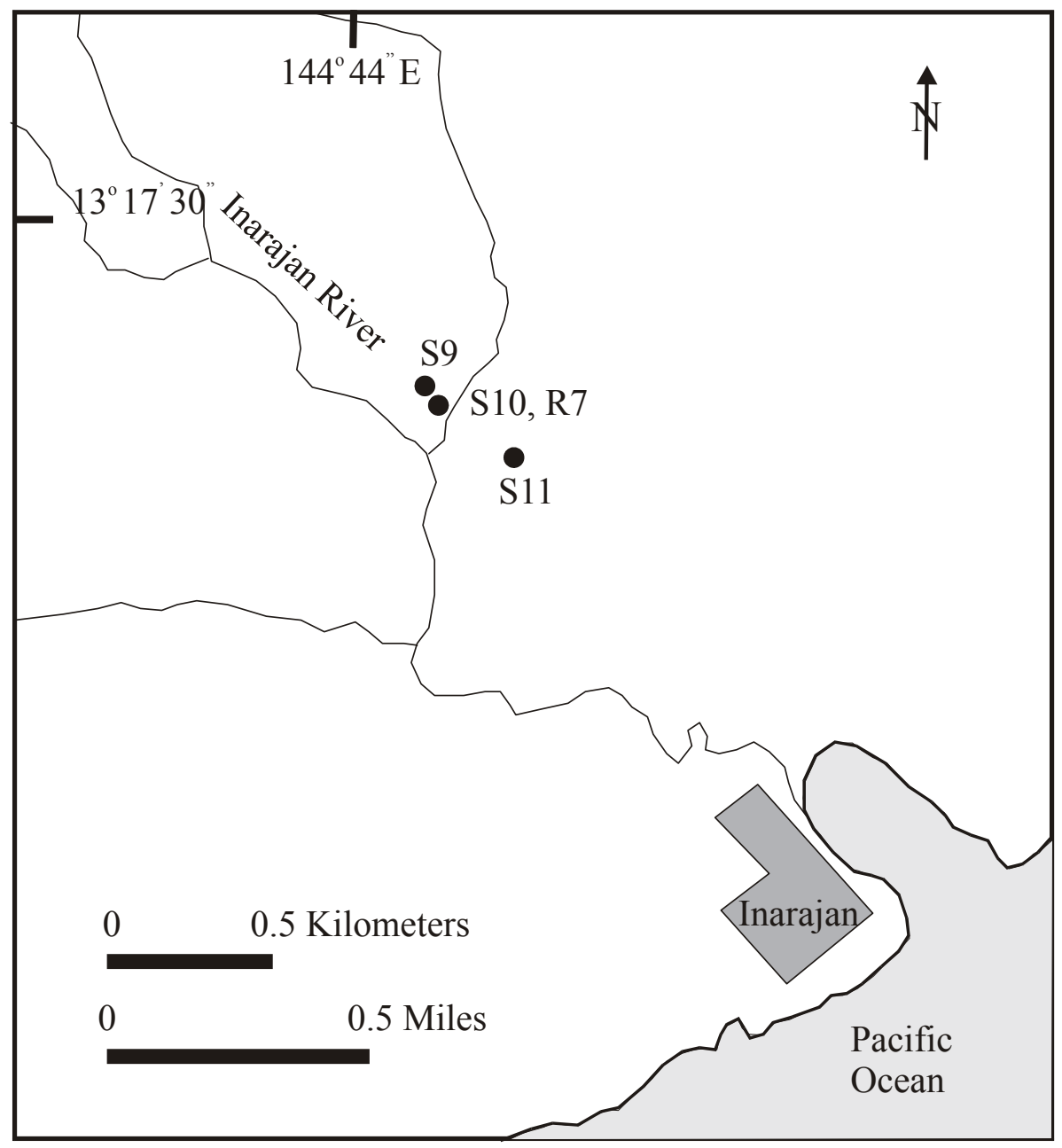

Figure 6. Map showing locations of sample sites within the Bolanos Formation in the vicinity of Inarajan. Sites prefixed with "S" are soils, "R" are rocks, and "SS" are stream sediments. 


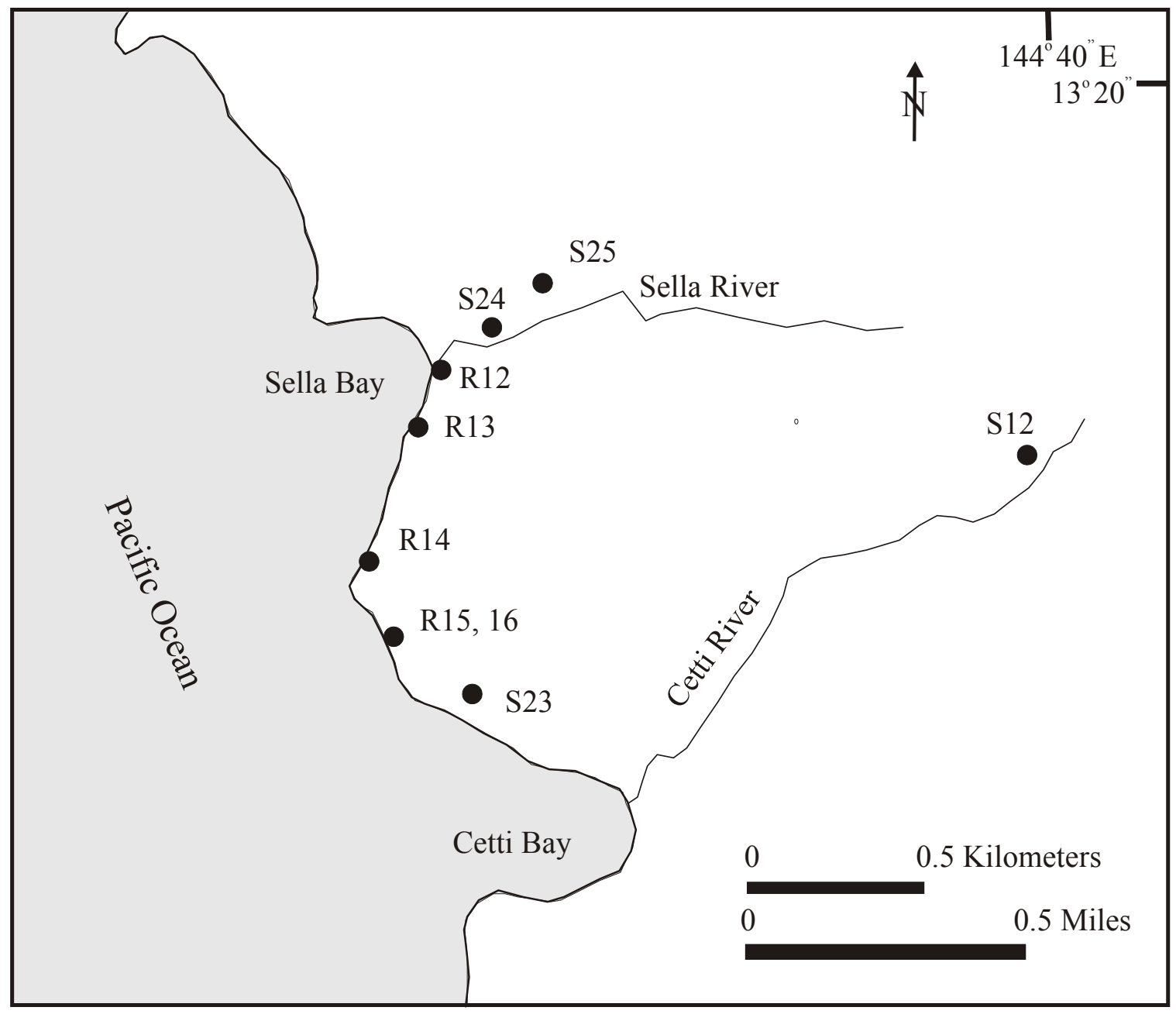

Figure 7. Map showing locations of sample sites within the Facpi Formation in the vicinity of Sella Bay and Cetti Bay. Sites prefixed with "S" are soils and "R" are rocks. 


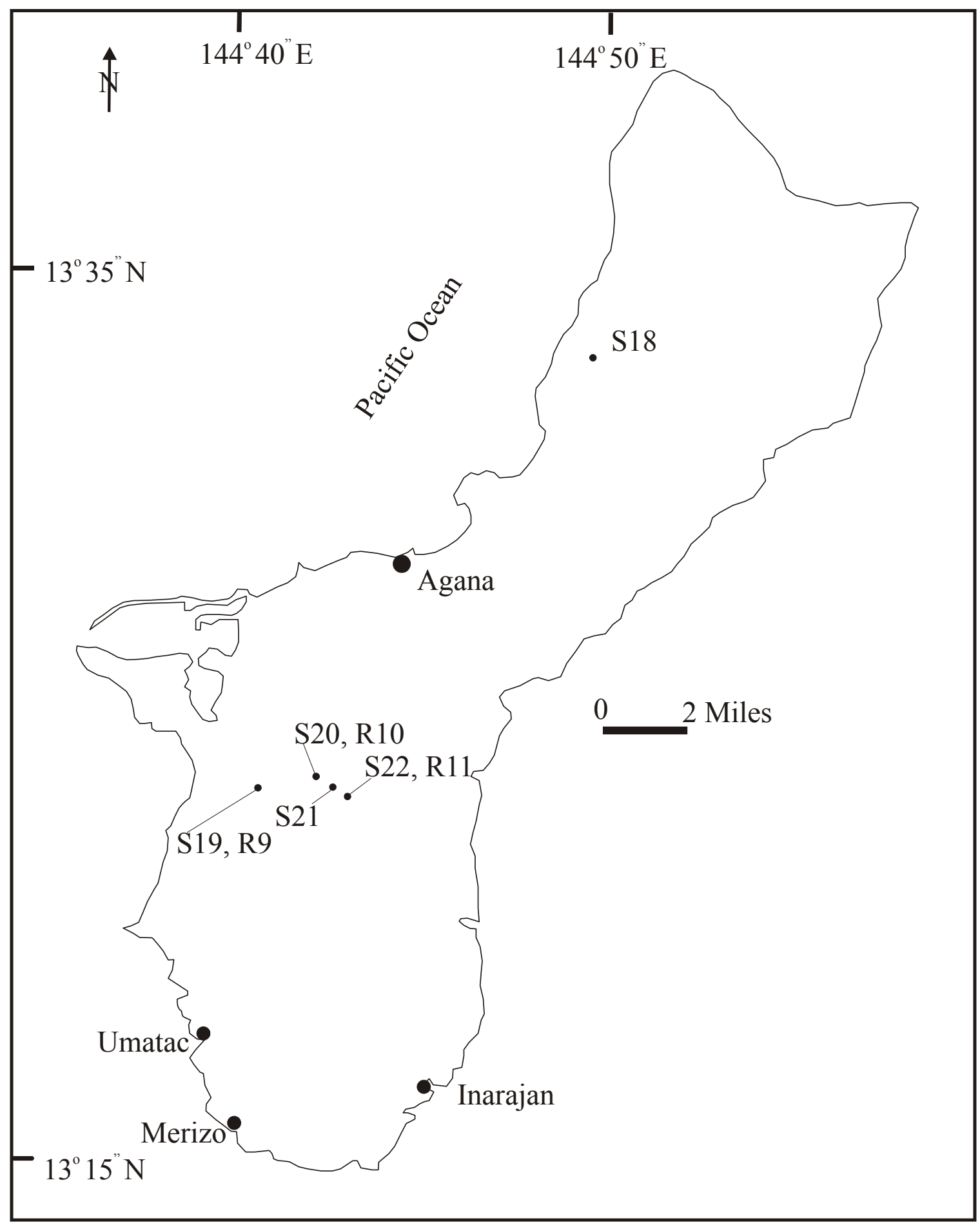

Figure 8. Map showing locations of sample sites within the Alutom Formation (except site $\mathrm{S} 18$ which is within the carbonate rocks) in the central part of Guam. Sites prefixed with " $S$ " are soils and " $\mathrm{R}$ " are rocks. 
determined, lower limits of determination, and analytical results for rock and soil samples are in tables 1-5.

Soil pH Determination: The $\mathrm{pH}$ was determined for soil samples by mixing $10 \mathrm{~g}$ of soil material with $20 \mathrm{~mL}$ of distilled and deionized water, letting the sample rest for 15 minutes and remixing. The $\mathrm{pH}$ was measured using a gel-filled epoxy electrode with automatic temperature compensation after letting the second sample mixing rest for an additional 15 minutes. Measurements were recorded on "ready" signal from the $\mathrm{pH}$ meter. Measurements were also made on replicate samples as well as with additional resting times (up to 90 minutes) and increased water volume $(40 \mathrm{~mL})$ with minimal (usually less than $0.1 \mathrm{pH}$ unit) differences noted in determinations.

Water Soluble Extraction: Soils were mixed with water to determine the water-soluble component of the samples. A $0.5 \mathrm{~g}$ sample of soil was placed into a $12 \mathrm{~mL}$ centrifuge tube and $5 \mathrm{~mL}$ of distilled and deionized water was added. The sample was shaken for one minute followed by centrifugation for 10 minutes at $15,000 \mathrm{rpm}$ using a refrigerated centrifuge set at $22{ }^{\circ} \mathrm{C}$. The procedure was selected to approximate previous work on Guam, Palau, and Jamaica to evaluate $\mathrm{Al}$ and $\mathrm{Ca}$ availability in soils (Crapper McLachlan and others, 1989). Results of the determination of 42 constituents (except some samples not analyzed for rare earth elements) by ICP-MS are shown in Table 6 and 7 . Results for duplicate analysis, in the tables, indicate good precision for the analysis.

Sequential Partial Dissolution Technique: A six-step sequential partial dissolution scheme was applied to selected soil samples to partition elements into operationally defined modes of occurrence or associations. The assessment of such techniques has been summarized by Tessier and others (1979), and Chao (1984), as furnishing information about the availability and mobility of elements in the environment. The scheme was applied to Guam soil samples to examine possible relative differences in the mode of occurrence of elements. Extractions were done sequentially in $50 \mathrm{~mL}$ polypropylene centrifuge tubes using a $0.25 \mathrm{~g}$ sample and $25 \mathrm{~mL}$ of extracting solution for each step. Shaking, when required, was done using a reciprocating shaker set at 130 strokes per minute. After each extraction, the solid and solution were separated by centrifuging at $15,000 \mathrm{rpm}$ for 10 minutes in a refrigerated centrifuge set at $22^{\circ} \mathrm{C}$. Subsequent steps used the separated residue from the previous step as the material to be extracted. Filter pulp $(0.25 \mathrm{~g})$ was added to the original $0.25 \mathrm{~g}$ sample in order to prevent caking of particles on the bottom of the centrifuge tube after centrifugation and to prevent particles from dislodging during decantation. Extracts were diluted 1:10 with deionized water prior to determination of selected elements by ICP-MS using matrix matching to address interelement interferences. The soil samples were extracted using the following stepwise procedures.

Fraction A - expected phase dissolution: water soluble, sorbed and exchangeable. Add $25 \mathrm{~mL}$ of 1 molar sodium acetate to a $0.25 \mathrm{~g}$ sample of soil (with $0.25 \mathrm{~g}$ filter pulp added) and shake at room temperature for 30 minutes. Centrifuge and decant solution into a 50 $\mathrm{mL}$ disposable polypropylene tube and add $0.5 \mathrm{~mL}$ of concentrated nitric acid to the tube. 
Chapman (1965) and Tessier and others (1979) discuss the use of sodium acetate as an extracting reagent.

Fraction B - expected phase dissolution: carbonates (Tessier and others, 1979). Add 25 $\mathrm{mL}$ of 1 molar sodium acetate adjusted to $\mathrm{pH} 5$ with acetic acid to the residue from fraction A. Shake at room temperature for 4 hours. Centrifuge and decant solution into a $50 \mathrm{~mL}$ disposable centrifuge tube.

Fraction C - expected phase dissolution: manganese oxides (Chao, 1972). Add $25 \mathrm{~mL}$ of 0.1 molar hydroxylamine hydrochloride in $0.01 \mathrm{~N}$ nitric acid to residue from fraction $\mathrm{B}$ and shake at room temperature for 30 minutes. Centrifuge and decant solution into a 50 $\mathrm{mL}$ disposable polypropylene tube and add $0.5 \mathrm{~mL}$ of concentrated nitric acid to the tube.

Fraction D - expected phase dissolution: amorphous iron oxides (Chao and Liyi, 1983). Add $25 \mathrm{~mL}$ of 0.25 molar hydroxylamine hydrochloride in $0.25 \mathrm{~N}$ hydrochloric acid to the residue from fraction $\mathrm{C}$ and place in a water bath at $50{ }^{\circ} \mathrm{C}$ for 30 minutes with occasional shaking. Centrifuge and decant solution into a $50 \mathrm{~mL}$ disposable centrifuge tube.

Fraction $\mathbf{E}$ - expected phase dissolution: crystalline iron and aluminum oxides and acid volatile sulfides (Chao and Sanzolone, 1989). Add $25 \mathrm{~mL}$ of $4 \mathrm{~N}$ hydrochloric acid to the residue from fraction $\mathrm{D}$ and place in a water bath at $95{ }^{\circ} \mathrm{C}$ for 45 minutes with occasional shaking. Centrifuge and decant solution into a $50 \mathrm{~mL}$ disposable centrifuge tube.

Fraction F - expected phase dissolution: residual (Chao and Sanzolone, 1989). Transfer the residue from fraction $\mathrm{E}$ to a $100 \mathrm{~mL}$ Teflon beaker using $5 \mathrm{~mL}$ of water. Place beaker on shaking hot plate under a perchloric acid hood and add $10 \mathrm{~mL}$ each of nitric, hydrofluoric, hydrochloric, and perchloric acid. Gradually increase temperature from 100 to $220^{\circ} \mathrm{C}$ over a three hour period and heat until the volume of the remaining acid is about $1 \mathrm{ml}$. Remove the beaker from the hot plate and add $10 \mathrm{~mL}$ of $50 \% \mathrm{v} / \mathrm{v}$ hydrochloric acid and return to hot plate for 5 minutes. Remove beaker from hot plate and allow cooling before transferring solution into a $50 \mathrm{~mL}$ polypropylene centrifuge tube and bringing the volume to $50 \mathrm{~mL}$ with water.

The results of the sequential partial extraction scheme are presented in Table 8. The precision of the extraction scheme can be evaluated by the use of a duplicate sample that is included in the table. Duplicate is generally within $+/-10 \%$ for each fraction for most elements. The reliability of the analysis can also be addressed by comparing the sum of the six fractions to the total element composition determined on a separate sample split. As shown in Table 8, agreement is usually good (+/-20\%) for most elements. Precision degrades, as expected, for elements with concentrations near or below the lower limits of determination for one or more fractions.

The sequential partial extraction scheme releases constituents in decreasing order of availability to the environment. Elements solubilized in extraction A are considered to be highly available to the environment; those released in $\mathrm{B}$ are considered to be available; those in $\mathrm{C}, \mathrm{D}$ and $\mathrm{E}$ are considered to be conditionally available (having the potential to 
become available through changes in Eh, $\mathrm{pH}$ or by microbial mobilization); and those in $\mathrm{F}$ are considered unavailable.

Simulated Lung Fluid Dissolution: The simulated lung fluid was adapted from that presented in Matson (1994) and Eastes and others (1995). This solution was selected because it provided an environment that is relevant to the extracellular fluid in the lung. The solution was made fresh the day before analysis in a metal-free environment. Onegram soil samples were weighed into $20 \mathrm{~mL}$ acid-washed scintillation vials equipped with a polyethylene mesh inserts. Twenty $\mathrm{mL}$ of simulated lung fluid were added to the vials to make a 1:20 mass/volume incubation ratio. Vials were placed into a Vitron Dynamic Organ Culture Incubator for $24 \mathrm{~h}$ at $37^{\circ} \mathrm{C}$. The incubator was implemented in order to keep samples in a constant rolling motion while holding the temperature constant. Following incubation the vials were centrifuged for $5 \mathrm{~min}$ at $15,000 \mathrm{rpm}$ and the simulated lung extract was then syringed filtered with a 0.45 -micron nitrocellulose filter. The filtered extract solution was analyzed by ICP-MS. Lung fluid reagent blanks limited the number of elements reported to 25 (Table 9). A duplicate lung fluid leach shown in Table 9 indicates good precision for the technique.

\section{Data}

The chemical analyses of the soils, rocks, and stream sediments from Guam are shown in Tables 1-3. The chemical analyses of soils and rocks from the western U. S. are shown in Tables 4 and 5 . The chemical analyses of the water soluble extractions of soils from Guam and the western U. S. are shown in Tables 6-7. The chemical analyses of the partial extraction scheme for soils from Guam and Colorado are shown in Table 8 and simulated lung fluid dissolution for soils from Guam and Colorado are in Table 9. 


\section{References}

Briggs, P.H. and Meier, A.L., 1999, The determination of forty two elements in Geological materials by inductively coupled plasma-mass spectrometry: U.S. Geological Survey Open-File Report 99-166, 15 p.

Carroll, D. and Hathaway, J.C., 1963, Mineralogy of selected soils from Guam: U.S. Geological Survey Professional Paper 403-F, 53 p.

Chao, T.T., 1972, Selective dissolution of manganese oxides from soils and sediments with acidified hydroxylamine hydrochloride: Soil Science Society of America Proceedings, v. 36, p. 764-768.

Chao, T.T., and Liyi, Zhou., 1983, Extraction techniques for selective dissolution of amorphous iron oxides from soils and sediments: Soil Science Society of America Journal, v. 47, no. 2, p. 225-232.

Chao, T.T., 1984, Use of partial dissolution techniques in geochemical exploration: Journal of Geochemical Exploration, v 20, p. 101-135.

Chao, T.T., and Sanzolone, R.F., 1989, Fractionation of soil selenium by sequential partial dissolution: Soil Science Society of America Journal, v. 53, no. 2, p. 385392.

Chapman, H.D., 1965, Methods of soil analysis: C.A. Black, Ed., American Society of Agronomy, Madison, Wisconsin., p. 891-904.

Crapper McLachlan, D.R., McLachlan, C.D., Krishnan, B., Krishnan, S.S., Dalton, A.J., and Steele, J.C., 1989, Aluminum and calcium in soil and food from Guam, Palau and Jamaica: Implications for amyotrophic lateral sclerosis and parkinsonismdementia syndromes of Guam: Environmental Geochemistry and Health v. 11, no. 2, p. 45-53.

Eastes, W. and Hadley, J. G., 1995, Dissolution of fibers inhaled by rats: Inhalation Toxicology, v. 7, p. 179-196.

Garruto, R.M., Yanagihara, R., Gajdusek, D.C., and Arion, D., 1984, Concentrations of heavy metals and essential minerals in garden soils and drinking water: In: Chen, L. and Yase, Y. (eds.), Western Pacific Amyotrophic Lateral Sclerosis in Asia and Oceania, National Taiwan Univ., Tapei, p. 265-330.

Koerrner, D.R., 1952, Amyotrophic lateral sclerosis on Guam: a clinical study and review of the literature: Annals of Internal Medicine, v. 37, p. 1204-1220. 
Kurland, L.T. and Mulder, D.W., 1954, Epidemiologic investigations of amyotrophic lateral sclerosis. 1. Preliminary report on geographic distribution with special reference to the Mariana Islands, including clinical and pathologic observations: Neurology, v. 4, p. 642-661.

MacDonald T.L. and Martin, R.B., 1988, Aluminum ion in biological systems: Trends Biochem., v. 13, P. 15-19.

Matson, S. M., 1994, Glass fibers in simulated lung fluid: dissolution behavior and analytical requirements: Annals of Occupational Hygiene, v. 38, p. 857-877.

Perl, D.P., Gajdusek, K.C., Garruto, R.M., Yanagihara, R.T., and Gibbs, C.J., 1982, Intraneuronal aluminum accumulation in amyotrophic lateral sclerosis and parkinsonism-dementia of Guam: Science, v. 217, p. 1053-1055.

Plato, C.C., Garruto, R.M., Fox, K.M., and Gajdusek, D.C., 1986, Amyotrophic lateral aclerosis and parkinsonism-dementia on Guam: a 25-year prospective casecontrol study: American Jour. Epidemiol, v. 124, p. 643-656.

Reagan, M.K. and Meijer, A., 1984, Geology and geochemistry of early arc-volcanic rocks from Guam: Geological Society of America, v. 95, p. 701-713.

Siegrist, H.G. and Randall, R.H., 1992, carbonate geology of Guam: $7^{\text {th }}$ International Coral Reef Symposium, Guam, U.S.A., June 18-20, 1992, 37 p.

Tessier, A., Campbell, P.G.C., and Bisson, M., 1979, Sequential extraction Procedure for the speciation of particulate trace metals: Analytical Chemistry, v. 51, no. 7, p. 844-851.

Tracey, J.I., Jr., Schlanger, S.O., Stark, J.T., Doan, D.B., and May, H.G., 1964, General Geology of Guam: U.S. Geological Survey Professional Paper 403-A, 104 p.

Young, F.J., 1988, Soil survey of the Territory of Guam: Washington, D.C., U. S. Soil Conservation Service, $166 \mathrm{p}$.

Zhang, Z.X., Anderson, K.W., and Mantel, N., 1990, Geographic patterns of parkinsonism-dementia complex on Guam, 1956 through 1985: Arch. Neurol. 1990, v. 47, p. 1069-1074. 
Table 1. Chemical analyses of soils from Guam

\begin{tabular}{ccccccc}
\hline Field No. & North Latitude & East Longitude & Formation & Al & Ca & Fe \\
\hline LLD & Degree & Degree & & $\%$ & $\%$ & $\%$ \\
GS01 & & & & 0.005 & 0.005 & 0.005 \\
GS02 & 13.2964 & 144.6742 & Facpi, tuff & 8.4 & 2.0 & 3.5 \\
GS03 & 13.2992 & 144.6811 & Facpi, tuff & 7.8 & 2.8 & 3.7 \\
GS04 & 13.2994 & 144.6806 & Facpi, tuff & 8.9 & 5.4 & 6.6 \\
GS05 & 13.3003 & 144.6794 & Facpi, tuff & 8.0 & 2.6 & 3.6 \\
GS06 & 13.2772 & 144.6853 & Facpi, tuff & 8.0 & 2.2 & 3.5 \\
GS07 & 13.2783 & 144.6856 & Facpi, tuff & 8.2 & 3.0 & 5.0 \\
GS08 & 13.2775 & 144.6856 & Facpi, tuff & 8.6 & 2.7 & 4.0 \\
GS09 & 13.2772 & 144.6853 & Facpi, tuff & 6.6 & 9.0 & 4.7 \\
GS10 & 13.2894 & 144.7372 & Bolanos & 7.3 & 5.7 & 5.2 \\
GS11 & 13.2878 & 144.7381 & Bolanos & 7.8 & 5.8 & 6.5 \\
GS12 & 13.2861 & 144.7408 & Bolanos & 8.2 & 4.6 & 6.2 \\
GS13 & 13.3258 & 144.6675 & Facpi, basalt & 8.0 & 3.5 & 7.3 \\
GS14 & 13.3003 & 144.6597 & Facpi, basalt & 6.3 & 8.2 & 3.6 \\
GS15 & 13.3022 & 144.6681 & Facpi, basalt & 8.8 & 4.9 & 6.3 \\
GS16 & 13.3031 & 144.6722 & Facpi, basalt & 7.6 & 1.7 & 4.0 \\
GS17 & 13.3033 & 144.6739 & Facpi, basalt & 7.1 & 1.1 & 3.3 \\
GS18 & 13.3033 & 144.6739 & Facpi, basalt & 7.8 & 1.5 & 3.7 \\
GS19 & 13.5606 & 144.8994 & Limestone, undivided & 26 & 1.2 & 14 \\
GS20 & 13.3903 & 144.6892 & Alutom & 7.4 & 12 & 5.3 \\
GS21 & 13.3936 & 144.7111 & Alutom & 15 & 0.20 & 12 \\
GS22 & 13.3908 & 144.7192 & Alutom & 11 & 0.59 & 8.5 \\
GS23 & 13.3889 & 144.7231 & Alutom & 14 & 0.2 & 13 \\
GS24 & 13.3175 & 144.6567 & Facpi, basalt & 8.2 & 3.1 & 7.4 \\
GS25 & 13.3278 & 144.6544 & Facpi, basalt & 7.3 & 2.3 & 7.7 \\
\hline & 13.3286 & 144.6550 & Facpi, basalt & 7.4 & 3.1 & 7.5 \\
\hline
\end{tabular}

${ }^{1}$ lower limit of determination 
Table 1. Chemical analyses of soils from Guam

\begin{tabular}{|c|c|c|c|c|c|c|c|c|c|}
\hline Field No. & $\mathbf{K}$ & Mg & $\mathbf{N a}$ & $\mathbf{P}$ & $\mathbf{T i}$ & Ag & As & $\mathbf{A u}$ & $\mathbf{B a}$ \\
\hline & $\%$ & $\%$ & $\%$ & $\%$ & $\%$ & $\mathrm{ppm}$ & $\mathrm{ppm}$ & $\mathrm{ppm}$ & $\mathrm{ppm}$ \\
\hline $\operatorname{LLD}^{1}$ & 0.005 & 0.005 & 0.005 & 0.01 & 0.005 & 3 & 0.5 & 0.05 & 0.5 \\
\hline GS01 & 3.0 & 0.82 & 2.0 & 0.11 & 0.50 & $<3$ & 4 & $<0.05$ & 1200 \\
\hline GS02 & 2.9 & 0.80 & 1.8 & 0.10 & 0.60 & $<3$ & 3 & $<0.05$ & 1100 \\
\hline GS03 & 1.3 & 2.3 & 1.9 & 0.18 & 1.0 & $<3$ & 2 & $<0.05$ & 1200 \\
\hline GS04 & 2.5 & 0.88 & 1.9 & 0.10 & 0.50 & $<3$ & 3 & $<0.05$ & 910 \\
\hline GS05 & 2.6 & 1.0 & 1.8 & 0.08 & 0.50 & $<3$ & 2 & $<0.05$ & 730 \\
\hline GS06 & 2.4 & 1.1 & 1.9 & 0.18 & 0.80 & $<3$ & 3 & $<0.05$ & 1300 \\
\hline GS07 & 2.4 & 1.3 & 2.1 & 0.10 & 0.60 & $<3$ & 2 & $<0.05$ & 1300 \\
\hline GS08 & 1.6 & 1.7 & 1.2 & 0.10 & 0.60 & $<3$ & 6.9 & $<0.05$ & 600 \\
\hline GS09 & 2.2 & 1.1 & 1.7 & 0.11 & 0.70 & $<3$ & 8 & $<0.05$ & 890 \\
\hline GS10 & 2.3 & 1.3 & 2.0 & 0.14 & 0.90 & $<3$ & 4 & $<0.05$ & 950 \\
\hline GS11 & 2.6 & 1.6 & 2.0 & 0.17 & 0.90 & $<3$ & 3 & $<0.05$ & 1100 \\
\hline GS12 & 2.7 & 1.3 & 2.2 & 0.19 & 1.1 & $<3$ & 2 & $<0.05$ & 960 \\
\hline GS13 & 1.9 & 1.1 & 1.3 & 0.17 & 0.70 & $<3$ & 4 & $<0.05$ & 920 \\
\hline GS14 & 2.4 & 2.0 & 3.1 & 0.42 & 1.3 & $<3$ & $<0.5$ & $<0.05$ & 1600 \\
\hline GS15 & 2.1 & 0.90 & 1.3 & 0.08 & 0.60 & $<3$ & 5 & $<0.05$ & 790 \\
\hline GS16 & 2.1 & 0.67 & 1.0 & 0.08 & 0.50 & $<3$ & 5.1 & $<0.05$ & 800 \\
\hline GS17 & 2.0 & 0.84 & 1.0 & 0.09 & 0.60 & $<3$ & 6.1 & $<0.05$ & 830 \\
\hline GS18 & 0.03 & 0.10 & 0.01 & 1.4 & 1.5 & $<3$ & 57 & $<0.05$ & 91 \\
\hline GS19 & 0.66 & 2.9 & 0.68 & 0.04 & 0.40 & $<3$ & $<0.5$ & $<0.05$ & 210 \\
\hline GS20 & 0.21 & 0.44 & 0.02 & 0.03 & 1.0 & $<3$ & 0.9 & $<0.05$ & 44 \\
\hline GS21 & 0.42 & 3.6 & 0.03 & 0.01 & 0.70 & $<3$ & $<0.5$ & $<0.05$ & 110 \\
\hline GS22 & 0.40 & 1.1 & 0.02 & 0.01 & 0.60 & $<3$ & 2 & $<0.05$ & 39 \\
\hline GS23 & 0.53 & 4.7 & 0.69 & 0.01 & 0.40 & $<3$ & $<0.5$ & $<0.05$ & 52 \\
\hline GS24 & 0.55 & 3.7 & 0.21 & 0.05 & 0.40 & $<3$ & 1 & $<0.05$ & 89 \\
\hline GS25 & 0.39 & 6.7 & 0.64 & $<0.01$ & 0.30 & $<3$ & $<0.5$ & $<0.05$ & 37 \\
\hline
\end{tabular}


Table 1. Chemical analyses of soils from Guam

\begin{tabular}{cccccccccc}
\hline Field No. & Be & Bi & Cd & Ce & Co & Cr & Cs & Cu & Dy \\
\hline LLD & ppm & ppm & ppm & ppm & ppm & ppm & ppm & ppm & ppm \\
GS01 & 0.1 & 0.05 & 0.1 & 0.5 & 0.1 & 0.2 & 0.01 & 3 & 0.04 \\
GS02 & 2.4 & 0.2 & 0.3 & 100 & 11 & 24 & 4 & 20 & 4.5 \\
GS03 & 1.9 & 0.1 & 0.2 & 88 & 13 & 39 & 3.6 & 20 & 4.5 \\
GS04 & 1.3 & 0.1 & 0.3 & 80 & 34 & 95 & 1.6 & 61 & 5.4 \\
GS05 & 2 & 0.1 & 0.2 & 74 & 12 & 35 & 2.8 & 20 & 4 \\
GS06 & 1.9 & 0.2 & 0.2 & 87 & 12 & 27 & 5.3 & 20 & 4.7 \\
GS07 & 2 & 0.1 & 0.3 & 94 & 24 & 79 & 2.7 & 52 & 4.7 \\
GS08 & 1.4 & 0.1 & 0.2 & 89 & 17 & 64 & 3 & 31 & 4.4 \\
GS09 & 1.8 & 0.1 & 0.2 & 70 & 16 & 36 & 3.1 & 20 & 4.4 \\
GS10 & 1.5 & 0.1 & 0.2 & 70 & 20 & 52 & 3 & 30 & 4.4 \\
GS11 & 2.3 & 0.1 & 0.2 & 97 & 26 & 72 & 4.4 & 75 & 4.9 \\
GS12 & 2 & 0.1 & 0.2 & 83 & 23 & 64 & 3.3 & 32 & 4.5 \\
GS13 & 1.6 & 0.1 & 0.3 & 88 & 15 & 30 & 3.4 & 30 & 5.1 \\
GS14 & 2.3 & $<0.05$ & 0.1 & 140 & 24 & 14 & 0.6 & 34 & 7.6 \\
GS15 & 2 & 0.2 & 0.3 & 79 & 22 & 68 & 4.5 & 35 & 4.8 \\
GS16 & 1.8 & 0.2 & 0.3 & 79 & 15 & 44 & 5.2 & 31 & 4.7 \\
GS17 & 2.3 & 0.2 & 0.3 & 93 & 16 & 46 & 5.3 & 30 & 5.4 \\
GS18 & 7 & 1.6 & 7.1 & 210 & 47 & 730 & 0.2 & 46 & 17 \\
GS19 & 0.4 & $<0.05$ & 0.4 & 9.9 & 33 & 150 & 0.3 & 78 & 4.9 \\
GS20 & 0.6 & $<0.05$ & $<0.1$ & 30 & 31 & 310 & 0.2 & 380 & 7.8 \\
GS21 & 0.5 & $<0.05$ & 0.4 & 13 & 50 & 280 & 0.2 & 110 & 4.5 \\
GS22 & 0.5 & $<0.05$ & 0.1 & 9.4 & 97 & 530 & 0.4 & 160 & 4.4 \\
GS23 & 0.7 & $<0.05$ & 0.2 & 6.5 & 52 & 570 & 0.4 & 96 & 2.8 \\
GS24 & 0.5 & $<0.05$ & 0.4 & 8.5 & 60 & 560 & 0.3 & 95 & 2.2 \\
GS25 & 0.4 & $<0.05$ & 0.1 & 4.9 & 58 & 620 & 0.4 & 74 & 2.3 \\
\hline & & & & & & & & 37 & 3.9 \\
\hline
\end{tabular}


Table 1. Chemical analyses of soils from Guam

\begin{tabular}{|c|c|c|c|c|c|c|c|c|c|}
\hline Field No. & $\mathbf{E r}$ & $\mathbf{E u}$ & $\mathbf{G a}$ & Gd & Ge & Ho & In & La & $\mathbf{L i}$ \\
\hline & $\mathrm{ppm}$ & $\mathrm{ppm}$ & ppm & $\mathrm{ppm}$ & $\mathrm{ppm}$ & $\mathrm{ppm}$ & $\mathrm{ppm}$ & ppm & ppm \\
\hline $\operatorname{LLD}^{1}$ & 0.05 & 0.005 & 0.006 & 0.02 & 0.1 & 0.02 & 0.1 & 0.3 & 0.2 \\
\hline GS01 & 2.2 & 1.4 & 18 & 5.3 & 1.3 & 0.69 & $<0.1$ & 53 & 24 \\
\hline GS02 & 2.3 & 1.4 & 17 & 5.1 & 1.2 & 0.72 & $<0.1$ & 44 & 23 \\
\hline GS03 & 2.7 & 1.8 & 19 & 6.2 & 1.2 & 0.86 & $<0.1$ & 39 & 18 \\
\hline GS04 & 2 & 1.2 & 17 & 4.7 & 1.2 & 0.64 & $<0.1$ & 39 & 24 \\
\hline GS05 & 2.5 & 1.3 & 17 & 5.2 & 1.2 & 0.75 & $<0.1$ & 47 & 26 \\
\hline GS06 & 2.3 & 1.6 & 19 & 5.8 & 1.1 & 0.74 & $<0.1$ & 46 & 24 \\
\hline GS07 & 2.2 & 1.5 & 19 & 5.1 & 1.4 & 0.69 & $<0.1$ & 46 & 26 \\
\hline GS08 & 2 & 1.1 & 14 & 4.3 & 0.9 & 0.62 & $<0.1$ & 30 & 21 \\
\hline GS09 & 2.2 & 1.3 & 17 & 5.1 & 1 & 0.7 & $<0.1$ & 38 & 27 \\
\hline GS10 & 2.2 & 1.5 & 18 & 5.1 & 1 & 0.7 & $<0.1$ & 39 & 18 \\
\hline GS11 & 2.4 & 1.6 & 19 & 5.9 & 1 & 0.77 & $<0.1$ & 50 & 23 \\
\hline GS12 & 2.2 & 1.5 & 20 & 5.5 & 1.1 & 0.7 & $<0.1$ & 45 & 18 \\
\hline GS13 & 2.6 & 1.5 & 14 & 6 & 1 & 0.82 & $<0.1$ & 46 & 23 \\
\hline GS14 & 3.7 & 2.7 & 20 & 9.3 & 0.8 & 1.2 & $<0.1$ & 65 & 13 \\
\hline GS15 & 2.4 & 1.3 & 16 & 5.3 & 1.2 & 0.75 & $<0.1$ & 40 & 27 \\
\hline GS16 & 2.2 & 1.2 & 15 & 5.3 & 1.1 & 0.72 & $<0.1$ & 40 & 29 \\
\hline GS17 & 2.6 & 1.5 & 17 & 6.4 & 1.2 & 0.83 & $<0.1$ & 48 & 31 \\
\hline GS18 & 9.2 & 4.3 & 37 & 18 & $<0.1$ & 2.9 & 0.3 & 120 & 7 \\
\hline GS19 & 3.1 & 0.96 & 12 & 4.2 & 1 & 0.93 & $<0.1$ & 8.1 & 17 \\
\hline GS20 & 4.7 & 1.7 & 29 & 6.3 & 1.9 & 1.4 & 0.1 & 11 & 5.5 \\
\hline GS21 & 2.8 & 0.9 & 19 & 3.6 & 1.4 & 0.81 & $<0.1$ & 5.4 & 37 \\
\hline GS22 & 3.1 & 0.7 & 25 & 2.8 & 2.1 & 0.84 & $<0.1$ & 2.6 & 22 \\
\hline GS23 & 1.7 & 0.5 & 14 & 2.1 & 1.1 & 0.52 & $<0.1$ & 3.3 & 29 \\
\hline GS24 & 1.3 & 0.48 & 13 & 1.7 & 1.2 & 0.38 & $<0.1$ & 3.8 & 31 \\
\hline GS25 & 1.5 & 0.43 & 12 & 1.7 & 1.4 & 0.43 & $<0.1$ & 2.5 & 46 \\
\hline
\end{tabular}


Table 1. Chemical analyses of soils from Guam

\begin{tabular}{|c|c|c|c|c|c|c|c|c|c|}
\hline Field No. & Mn & Mo & $\mathrm{Nb}$ & Nd & $\mathbf{N i}$ & $\mathbf{P b}$ & Pr & $\mathbf{R b}$ & $\mathbf{R e}$ \\
\hline & $\mathrm{ppm}$ & ppm & ppm & ppm & $\mathrm{ppm}$ & $\mathrm{ppm}$ & $\mathrm{ppm}$ & ppm & $\mathrm{ppm}$ \\
\hline $\operatorname{LLD}^{1}$ & 0.2 & 0.1 & 2 & 0.2 & 1 & 0.8 & 0.05 & 0.01 & 0.05 \\
\hline GS01 & 1300 & 1.3 & 21 & 42 & 12 & 26 & 11 & 100 & $<0.05$ \\
\hline GS02 & 1000 & 1.2 & 18 & 36 & 16 & 22 & 9 & 100 & $<0.05$ \\
\hline GS03 & 1400 & 0.6 & 14 & 40 & 44 & 18 & 9.3 & 42 & $<0.05$ \\
\hline GS04 & 820 & 1.1 & 14 & 33 & 14 & 21 & 8.2 & 85 & $<0.05$ \\
\hline GS05 & 890 & 1.6 & 20 & 38 & 13 & 24 & 9.6 & 110 & $<0.05$ \\
\hline GS06 & 1800 & 0.7 & 18 & 42 & 42 & 22 & 9.9 & 72 & $<0.05$ \\
\hline GS07 & 1200 & 0.9 & 18 & 38 & 28 & 20 & 9.5 & 83 & $<0.05$ \\
\hline GS08 & 890 & 0.8 & 12 & 27 & 39 & 13 & 6.5 & 64 & $<0.05$ \\
\hline GS09 & 900 & 1.1 & 14 & 34 & 19 & 15 & 8.1 & 74 & $<0.05$ \\
\hline GS10 & 1000 & 1.3 & 14 & 35 & 22 & 16 & 8.4 & 72 & $<0.05$ \\
\hline GS11 & 1400 & 1.2 & 18 & 43 & 49 & 22 & 11 & 79 & $<0.05$ \\
\hline GS12 & 1200 & 1.3 & 18 & 38 & 28 & 19 & 9.3 & 84 & $<0.05$ \\
\hline GS13 & 780 & 0.8 & 20 & 41 & 21 & 16 & 9.9 & 68 & $<0.05$ \\
\hline GS14 & 1000 & 0.9 & 29 & 67 & 19 & 11 & 16 & 44 & $<0.05$ \\
\hline GS15 & 1100 & 0.9 & 17 & 34 & 44 & 20 & 8.5 & 93 & $<0.05$ \\
\hline GS16 & 940 & 1 & 16 & 35 & 23 & 20 & 8.5 & 110 & $<0.05$ \\
\hline GS17 & 860 & 1 & 19 & 42 & 26 & 22 & 10 & 96 & $<0.05$ \\
\hline GS18 & 5600 & 4.3 & 29 & 100 & 320 & 77 & 25 & 0.5 & $<0.05$ \\
\hline GS19 & 1400 & 0.2 & 1.1 & 11 & 100 & 3.6 & 2.1 & 14 & $<0.05$ \\
\hline GS20 & 940 & 0.7 & 2.3 & 21 & 140 & 5.6 & 4.1 & 7.4 & $<0.05$ \\
\hline GS21 & 1800 & 0.2 & 1.5 & 9.5 & 150 & 2 & 1.8 & 13 & $<0.05$ \\
\hline GS22 & 2300 & 0.6 & 3.9 & 5.5 & 380 & 2 & 0.98 & 15 & $<0.05$ \\
\hline GS23 & 1300 & 0.3 & 2.5 & 5 & 270 & 6.1 & 0.94 & 12 & $<0.05$ \\
\hline GS24 & 1400 & 0.7 & 2.3 & 5.1 & 240 & 3.6 & 1 & 21 & $<0.05$ \\
\hline GS25 & 1300 & 0.1 & 1.9 & 4 & 310 & $<0.8$ & 0.71 & 9.2 & $<0.05$ \\
\hline
\end{tabular}


Table 1. Chemical analyses of soils from Guam

\begin{tabular}{|c|c|c|c|c|c|c|c|c|c|}
\hline Field No. & $\mathbf{S b}$ & Sc & Se & Sm & Sn & $\mathrm{Sr}$ & $\mathbf{T b}$ & Te & Th \\
\hline & $\mathrm{ppm}$ & $\mathrm{ppm}$ & $\mathrm{ppm}$ & $\mathrm{ppm}$ & ppm & $\mathrm{ppm}$ & $\mathrm{ppm}$ & $\mathrm{ppm}$ & ppm \\
\hline $\operatorname{LLD}^{1}$ & 0.1 & 0.3 & 1 & 0.02 & 0.5 & 0.05 & 0.005 & 0.1 & 0.03 \\
\hline GS01 & 0.6 & 8.8 & $<1$ & 6.9 & 3 & 500 & 0.67 & $<0.1$ & 9.9 \\
\hline GS02 & 0.6 & 9.7 & $<1$ & 6.1 & 2 & 390 & 0.65 & $<0.1$ & 10 \\
\hline GS03 & 0.3 & 23 & $<1$ & 7.2 & 2 & 850 & 0.79 & $<0.1$ & 4.7 \\
\hline GS04 & 0.5 & 9.6 & $<1$ & 5.4 & 2 & 490 & 0.6 & $<0.1$ & 8 \\
\hline GS05 & 0.6 & 10 & $<1$ & 6.6 & 2 & 380 & 0.71 & $<0.1$ & 12 \\
\hline GS06 & 0.5 & 16 & $<1$ & 7.1 & 2 & 680 & 0.7 & $<0.1$ & 6.9 \\
\hline GS07 & 0.5 & 12 & $<1$ & 6.3 & 2 & 580 & 0.65 & $<0.1$ & 7.8 \\
\hline GS08 & 0.7 & 13 & $<1$ & 4.9 & 1 & 350 & 0.59 & $<0.1$ & 6.3 \\
\hline GS09 & 0.7 & 11 & $<1$ & 5.7 & 2 & 510 & 0.64 & $<0.1$ & 7.9 \\
\hline GS10 & 0.9 & 12 & $<1$ & 6.3 & 2 & 620 & 0.68 & $<0.1$ & 6.7 \\
\hline GS11 & 0.7 & 14 & $<1$ & 7.1 & 2 & 750 & 0.72 & $<0.1$ & 12 \\
\hline GS12 & 0.6 & 13 & $<1$ & 6.4 & 2 & 680 & 0.69 & $<0.1$ & 8.4 \\
\hline GS13 & 0.7 & 9.9 & $<1$ & 7 & 2 & 440 & 0.78 & $<0.1$ & 7.9 \\
\hline GS14 & $<0.1$ & 16 & $<1$ & 11 & 2 & 1000 & 1.2 & $<0.1$ & 2.9 \\
\hline GS15 & 0.7 & 12 & $<1$ & 6.1 & 2 & 270 & 0.71 & $<0.1$ & 9.3 \\
\hline GS16 & 0.8 & 10 & $<1$ & 6.2 & 2 & 200 & 0.68 & $<0.1$ & 9.6 \\
\hline GS17 & 0.8 & 11 & $<1$ & 7.4 & 2 & 230 & 0.82 & $<0.1$ & 10 \\
\hline GS18 & 14 & 50 & $<1$ & 19 & 7.8 & 1900 & 2.4 & 0.8 & 32 \\
\hline GS19 & 0.4 & 30 & $<1$ & 2.8 & 0.7 & 160 & 0.65 & $<0.1$ & 0.37 \\
\hline GS20 & 0.3 & 71 & $<1$ & 5.7 & 1 & 49 & 1 & $<0.1$ & 0.89 \\
\hline GS21 & 0.2 & 49 & $<1$ & 2.7 & 0.8 & 70 & 0.58 & $<0.1$ & 0.4 \\
\hline GS22 & 0.3 & 76 & $<1$ & 1.9 & 0.9 & 12 & 0.51 & $<0.1$ & 0.59 \\
\hline GS23 & $<0.1$ & 42 & $<1$ & 1.5 & 0.8 & 100 & 0.34 & $<0.1$ & 0.38 \\
\hline GS24 & 0.3 & 39 & $<1$ & 1.4 & 0.6 & 140 & 0.28 & $<0.1$ & 0.38 \\
\hline GS25 & $<0.1$ & 45 & $<1$ & 1.2 & $<0.5$ & 150 & 0.28 & $<0.1$ & 0.25 \\
\hline
\end{tabular}


Table 1. Chemical analyses of soils from Guam

\begin{tabular}{|c|c|c|c|c|c|c|c|c|}
\hline Field No. & $\mathbf{T l}$ & $\mathbf{T m}$ & $\mathbf{U}$ & $\mathbf{V}$ & $\mathbf{W}$ & $\mathbf{Y}$ & $\mathbf{Y b}$ & $\mathbf{Z n}$ \\
\hline & $\mathrm{ppm}$ & $\mathrm{ppm}$ & ppm & ppm & $\mathrm{ppm}$ & ppm & ppm & ppm \\
\hline $\operatorname{LLD}^{1}$ & 0.1 & 0.006 & 0.02 & 0.4 & 0.1 & 0.3 & 0.03 & 5 \\
\hline GS01 & 0.6 & 0.3 & 2.5 & 74 & 1.4 & 33 & 2.1 & 110 \\
\hline GS02 & 0.5 & 0.32 & 2.6 & 92 & 1.3 & 34 & 2.2 & 110 \\
\hline GS03 & 0.2 & 0.36 & 1.2 & 200 & 0.6 & 41 & 2.5 & 110 \\
\hline GS04 & 0.4 & 0.28 & 2.1 & 88 & 1 & 30 & 1.9 & 81 \\
\hline GS05 & 0.7 & 0.34 & 3.8 & 80 & 1.2 & 37 & 2.4 & 94 \\
\hline GS06 & 0.4 & 0.31 & 1.7 & 110 & 1.6 & 34 & 2 & 100 \\
\hline GS07 & 0.5 & 0.3 & 2.2 & 99 & 1 & 33 & 2 & 97 \\
\hline GS08 & 0.4 & 0.27 & 1.6 & 120 & 0.9 & 30 & 1.9 & 86 \\
\hline GS09 & 0.4 & 0.3 & 3.4 & 150 & 1.3 & 34 & 2 & 110 \\
\hline GS10 & 0.6 & 0.3 & 2.1 & 180 & 1 & 34 & 2 & 130 \\
\hline GS11 & 0.6 & 0.32 & 2.7 & 170 & 1.4 & 37 & 2.2 & 120 \\
\hline GS12 & 0.5 & 0.29 & 2.3 & 210 & 1.1 & 34 & 2 & 140 \\
\hline GS13 & 0.4 & 0.34 & 1.8 & 81 & 1.1 & 39 & 2.3 & 79 \\
\hline GS14 & 0.1 & 0.48 & 0.88 & 140 & 0.4 & 57 & 3.2 & 120 \\
\hline GS15 & 0.6 & 0.31 & 2.3 & 99 & 1.1 & 36 & 2.1 & 87 \\
\hline GS16 & 0.6 & 0.3 & 2.5 & 76 & 1.2 & 34 & 2.1 & 88 \\
\hline GS17 & 0.6 & 0.35 & 2.3 & 84 & 1.2 & 40 & 2.4 & 93 \\
\hline GS18 & 0.6 & 1.2 & 17 & 510 & 5.1 & 200 & 8.3 & 57 \\
\hline GS19 & $<0.1$ & 0.42 & 0.5 & 180 & 0.3 & 54 & 2.9 & 77 \\
\hline GS20 & $<0.1$ & 0.66 & 1.3 & 540 & 0.2 & 64 & 4.8 & 81 \\
\hline GS21 & $<0.1$ & 0.39 & 0.4 & 280 & 0.1 & 38 & 2.8 & 100 \\
\hline GS22 & $<0.1$ & 0.46 & 0.4 & 280 & 0.2 & 38 & 3.4 & 97 \\
\hline GS23 & $<0.1$ & 0.25 & 0.2 & 180 & $<0.1$ & 23 & 1.8 & 70 \\
\hline GS24 & $<0.1$ & 0.19 & 0.4 & 190 & $<0.1$ & 17 & 1.4 & 92 \\
\hline GS25 & $<0.1$ & 0.21 & 0.06 & 160 & $<0.1$ & 20 & 1.5 & 72 \\
\hline
\end{tabular}


Table 2. Chemical analyses of rocks from Guam

\begin{tabular}{ccclcccc}
\hline Field No. & $\begin{array}{c}\text { North Latitude } \\
\text { Degree }\end{array}$ & $\begin{array}{c}\text { East Longitude } \\
\text { Degree }\end{array}$ & Formation & $\begin{array}{c}\text { Al } \\
\%\end{array}$ & $\begin{array}{c}\text { Ca } \\
\%\end{array}$ & $\begin{array}{c}\text { Fe } \\
\%\end{array}$ & $\begin{array}{c}\text { K } \\
\%\end{array}$ \\
\hline \hline LLD & & & & 0.005 & 0.005 & 0.005 & 0.005 \\
GR01 & 13.2992 & 144.6811 & Facpi, tuff & 4.5 & 21 & 3.2 & 0.42 \\
GR02 & 13.2994 & 144.6806 & Facpi, tuff & 1.2 & 34 & 0.95 & 0.17 \\
GR03 & 13.2989 & 144.6792 & Facpi, tuff & 1.4 & 28 & 1.4 & 0.13 \\
GR04 & 13.2964 & 144.6767 & Facpi, tuff & 1.6 & 35 & 0.90 & 0.29 \\
GR05 & 13.2772 & 144.6853 & Facpi, tuff & 3.1 & 29 & 2.0 & 0.48 \\
GR07 & 13.2878 & 144.7381 & Bolonas & 7.8 & 3.4 & 5.6 & 1.4 \\
GR08 & 13.3033 & 144.6739 & Facpi, basalt & 7.2 & 5.8 & 6.1 & 1.2 \\
GR09 & 13.3903 & 144.6892 & Alutom & 4.6 & 17 & 3.2 & 0.60 \\
GR10 & 13.3936 & 144.7111 & Alutom & $>20$ & 0.2 & 7.7 & 0.60 \\
GR11 & 13.3889 & 144.7231 & Alutom & 9.8 & 0.07 & 8.4 & 0.42 \\
GR12 & 13.3278 & 144.6519 & Facpi, veins & 1.4 & 6.2 & 0.58 & 0.38 \\
GR13 & 13.3250 & 144.6528 & Facpi, veins & 3 & 33 & 2.6 & 0.21 \\
GR14 & 13.3203 & 144.6522 & Facpi, veins & 4.6 & 27 & 4.3 & 0.51 \\
GR15 & 13.3175 & 144.6564 & Facpi, basalt & 7 & 17 & 5.4 & 0.53 \\
GR16 & 13.3175 & 144.6564 & Facpi, veins & 1.2 & 42 & 2.4 & 0.57 \\
\hline
\end{tabular}

${ }^{1}$ lower limit of determination 
Table 2. Chemical analyses of rocks from Guam

\begin{tabular}{cccccccccc}
\hline Field No. & $\begin{array}{c}\mathrm{Mg} \\
\%\end{array}$ & $\begin{array}{c}\mathrm{Na} \\
\%\end{array}$ & $\begin{array}{c}\mathrm{P} \\
\%\end{array}$ & $\begin{array}{c}\mathrm{Ti} \\
\%\end{array}$ & $\begin{array}{c}\mathrm{Ag} \\
\mathrm{ppm}\end{array}$ & $\begin{array}{c}\mathrm{As} \\
\mathrm{ppm}\end{array}$ & $\begin{array}{c}\mathrm{Au} \\
\mathrm{ppm}\end{array}$ & $\begin{array}{c}\mathrm{Ba} \\
\mathrm{ppm}\end{array}$ & $\begin{array}{c}\mathrm{Be} \\
\mathrm{ppm}\end{array}$ \\
\hline \hline LLD $^{1}$ & 0.005 & 0.005 & 0.01 & 0.005 & 3 & 0.5 & 0.05 & 0.5 & 0.1 \\
GR01 & 1.4 & 0.18 & 0.03 & 0.30 & $<3$ & 2 & $<0.05$ & 180 & 0.3 \\
GR02 & 0.43 & 0.04 & 0.03 & 0.08 & $<3$ & $<0.5$ & $<0.05$ & 160 & 0.2 \\
GR03 & 0.62 & 0.16 & 0.03 & 0.10 & $<3$ & $<0.5$ & $<0.05$ & 190 & 0.2 \\
GR04 & 0.55 & 0.17 & 0.02 & 0.10 & $<3$ & $<0.5$ & $<0.05$ & 78 & $<0.1$ \\
GR05 & 1.3 & 0.18 & 0.03 & 0.20 & $<3$ & $<0.5$ & $<0.05$ & 45 & 0.1 \\
GR07 & 3.1 & 0.78 & 0.05 & 0.50 & $<3$ & $<0.5$ & $<0.05$ & 230 & 0.6 \\
GR08 & 5.1 & 0.46 & $<0.01$ & 0.30 & $<3$ & $<0.5$ & $<0.05$ & 22 & 0.3 \\
GR09 & 2.6 & 0.59 & 0.03 & 0.30 & $<3$ & $<0.5$ & $<0.05$ & 55 & 0.3 \\
GR10 & 2.0 & 0.09 & $<0.01$ & 0.80 & $<3$ & $<0.5$ & $<0.05$ & 170 & 1.2 \\
GR11 & 2.1 & 0.03 & $<0.01$ & 0.40 & $<3$ & $<0.5$ & $<0.05$ & 49 & 0.9 \\
GR12 & 0.43 & 0.50 & 0.01 & 0.05 & $<3$ & $<0.5$ & $<0.05$ & 41 & 0.5 \\
GR13 & 3.4 & 0.98 & 0.04 & 0.10 & $<3$ & $<0.5$ & $<0.05$ & 11 & 0.2 \\
GR14 & 3.8 & 1.1 & 0.04 & 0.20 & $<3$ & 0.6 & $<0.05$ & 31 & 0.5 \\
GR15 & 4.4 & 1.6 & 0.04 & 0.40 & $<3$ & $<0.5$ & $<0.05$ & 49 & 0.7 \\
GR16 & 1.5 & 0.26 & 0.04 & 0.06 & $<3$ & $<0.5$ & $<0.05$ & 7 & 0.2 \\
\hline
\end{tabular}


Table 2. Chemical analyses of rocks from Guam

\begin{tabular}{cccccccccc}
\hline Field No. & $\begin{array}{c}\mathrm{Bi} \\
\mathrm{ppm}\end{array}$ & $\begin{array}{c}\mathrm{Cd} \\
\mathrm{ppm}\end{array}$ & $\begin{array}{c}\mathrm{Ce} \\
\mathrm{ppm}\end{array}$ & $\begin{array}{c}\mathrm{Co} \\
\mathrm{ppm}\end{array}$ & $\begin{array}{c}\mathrm{Cr} \\
\mathrm{ppm}\end{array}$ & $\begin{array}{c}\mathrm{Cs} \\
\mathrm{ppm}\end{array}$ & $\begin{array}{c}\mathrm{Cu} \\
\mathrm{ppm}\end{array}$ & $\begin{array}{c}\text { Dy } \\
\mathrm{ppm}\end{array}$ & $\begin{array}{c}\text { Er } \\
\mathrm{ppm}\end{array}$ \\
\hline \hline LLD $^{1}$ & 0.05 & 0.1 & 0.5 & 0.1 & 0.2 & 0.01 & 3 & 0.04 & 0.05 \\
GR01 & $<0.07$ & $<0.1$ & 6.2 & 16 & 97 & 0.4 & 54 & 1.7 & 1.0 \\
GR02 & $<0.07$ & 0.2 & 1.9 & 4.3 & 24 & 0.1 & 20 & 0.92 & 0.54 \\
GR03 & $<0.07$ & $<0.1$ & 2.4 & 6.8 & 48 & 0.1 & 60 & 1.4 & 0.76 \\
GR04 & $<0.07$ & 0.2 & 1.9 & 3.8 & 54 & $<0.1$ & 9 & 0.66 & 0.41 \\
GR05 & $<0.07$ & 0.2 & 5.3 & 8.2 & 30 & 0.2 & 20 & 1.4 & 0.88 \\
GR07 & $<0.07$ & $<0.1$ & 14 & 25 & 80 & 0.3 & 110 & 2.9 & 1.6 \\
GR08 & $<0.07$ & 0.2 & 5.5 & 49 & 830 & 0.1 & 63 & 2.4 & 1.4 \\
GR09 & $<0.07$ & 0.1 & 4.3 & 18 & 100 & 0.2 & 39 & 1.6 & 1.0 \\
GR10 & $<0.07$ & $<0.1$ & 21 & 120 & 120 & 0.3 & 430 & 10 & 5.9 \\
GR11 & $<0.07$ & 0.2 & 4.8 & 52 & 400 & 0.2 & 140 & 3.1 & 2.1 \\
GR12 & $<0.07$ & 0.1 & 3.1 & 2.5 & 29 & $<0.1$ & 260 & 0.71 & 0.53 \\
GR13 & $<0.07$ & 0.8 & 1.6 & 20 & 160 & $<0.1$ & 44 & 1.1 & 0.85 \\
GR14 & $<0.07$ & 0.7 & 4.3 & 26 & 260 & 0.2 & 110 & 1.4 & 0.98 \\
GR15 & $<0.07$ & 0.4 & 6.2 & 33 & 270 & 0.3 & 81 & 2.1 & 1.3 \\
GR16 & $<0.07$ & 0.6 & 1.0 & 10 & 52 & 0.3 & 94 & 0.38 & 0.26 \\
\hline
\end{tabular}


Table 2. Chemical analyses of rocks from Guam

\begin{tabular}{cccccccccc}
\hline Field No. & $\begin{array}{c}\text { Eu } \\
\text { ppm }\end{array}$ & $\begin{array}{c}\text { Ga } \\
\text { ppm }\end{array}$ & $\begin{array}{c}\text { Gd } \\
\text { ppm }\end{array}$ & $\begin{array}{c}\text { Ge } \\
\text { ppm }\end{array}$ & $\begin{array}{c}\text { Ho } \\
\text { ppm }\end{array}$ & $\begin{array}{c}\text { In } \\
\text { ppm }\end{array}$ & $\begin{array}{c}\text { La } \\
\text { ppm }\end{array}$ & $\begin{array}{c}\text { Li } \\
\text { ppm }\end{array}$ & $\begin{array}{c}\text { Mn } \\
\text { ppm }\end{array}$ \\
\hline \hline LLD $^{1}$ & 0.005 & 0.006 & 0.02 & 0.1 & 0.02 & 0.1 & 0.3 & 0.2 & 0.2 \\
GR01 & 0.37 & 7.6 & 1.5 & 0.7 & 0.31 & $<0.1$ & 6.0 & 38 & 790 \\
GR02 & 0.19 & 2.1 & 0.86 & 0.2 & 0.17 & $<0.1$ & 4.4 & 5.9 & 1000 \\
GR03 & 0.28 & 2.5 & 1.3 & 0.3 & 0.25 & $<0.1$ & 6.2 & 13 & 1400 \\
GR04 & 0.14 & 2.4 & 0.54 & 0.2 & 0.12 & $<0.1$ & 1.6 & 4.0 & 830 \\
GR05 & 0.29 & 4.7 & 1.2 & 0.4 & 0.26 & $<0.1$ & 4.5 & 18 & 530 \\
GR07 & 0.74 & 12 & 2.7 & 1.2 & 0.51 & $<0.1$ & 8.7 & 12 & 420 \\
GR08 & 0.48 & 9.2 & 2 & 1.2 & 0.44 & $<0.1$ & 4.5 & 16 & 1100 \\
GR09 & 0.34 & 7.0 & 1.3 & 0.7 & 0.30 & $<0.1$ & 2.1 & 8 & 720 \\
GR10 & 2.4 & 23 & 9.8 & 1.7 & 1.9 & $<0.1$ & 16 & 12 & 1700 \\
GR11 & 0.54 & 16 & 2.3 & 1.7 & 0.60 & $<0.1$ & 4.1 & 45 & 1300 \\
GR12 & 0.11 & 4.1 & 0.53 & 1.2 & 0.14 & $<0.1$ & 2.4 & 5.0 & 290 \\
GR13 & 0.17 & 4.7 & 0.76 & 0.6 & 0.23 & $<0.1$ & 1.7 & 26 & 560 \\
GR14 & 0.25 & 8.2 & 1.0 & 0.9 & 0.28 & $<0.1$ & 2.8 & 24 & 1200 \\
GR15 & 0.38 & 11 & 1.6 & 0.9 & 0.39 & $<0.1$ & 3.2 & 10 & 940 \\
GR16 & 0.06 & 2.2 & 0.25 & 0.8 & 0.08 & $<0.1$ & 0.70 & 5.7 & 520 \\
\hline
\end{tabular}

${ }^{1}$ lower limit of determination 
Table 2. Chemical analyses of rocks from Guam

\begin{tabular}{cccccccccc}
\hline Field No. & $\begin{array}{c}\text { Mo } \\
\text { ppm }\end{array}$ & $\begin{array}{c}\mathrm{Nb} \\
\mathrm{ppm}\end{array}$ & $\begin{array}{c}\mathrm{Nd} \\
\mathrm{ppm}\end{array}$ & $\begin{array}{c}\mathrm{Ni} \\
\mathrm{ppm}\end{array}$ & $\begin{array}{c}\mathrm{Pb} \\
\mathrm{ppm}\end{array}$ & $\begin{array}{c}\mathrm{Pr} \\
\mathrm{ppm}\end{array}$ & $\begin{array}{c}\mathrm{Rb} \\
\mathrm{ppm}\end{array}$ & $\begin{array}{c}\mathrm{Re} \\
\mathrm{ppm}\end{array}$ & $\begin{array}{c}\mathrm{Sb} \\
\mathrm{ppm}\end{array}$ \\
\hline \hline LLD $^{1}$ & 0.1 & 2.0 & 0.2 & 1 & 0.8 & 0.05 & 0.01 & 0.05 & 0.1 \\
GR01 & 0.3 & 1.2 & 5.9 & 56 & 2.1 & 1.3 & 11 & $<0.05$ & 0.2 \\
GR02 & $<0.1$ & 0.40 & 3.8 & 16 & 0.8 & 0.82 & 3.6 & $<0.05$ & $<0.1$ \\
GR03 & $<0.1$ & 0.60 & 5.5 & 40 & 1.9 & 1.2 & 3.5 & $<0.05$ & $<0.1$ \\
GR04 & 0.1 & 0.20 & 1.7 & 12 & 0.5 & 0.40 & 3.5 & $<0.05$ & 0.1 \\
GR05 & $<0.1$ & 0.89 & 4.6 & 14 & 1.3 & 0.98 & 26 & $<0.05$ & $<0.1$ \\
GR07 & $<0.1$ & 2.4 & 11 & 26 & 1.9 & 2.4 & 33 & $<0.05$ & $<0.1$ \\
GR08 & 0.2 & 1.6 & 5.3 & 230 & 0.9 & 1.1 & 19 & $<0.05$ & 0.2 \\
GR09 & 0.1 & 0.50 & 3.5 & 58 & 0.8 & 0.67 & 9.8 & $<0.05$ & $<0.1$ \\
GR10 & $<0.1$ & 2.1 & 28 & 77 & 3.2 & 5.5 & 5.0 & $<0.05$ & 0.1 \\
GR11 & 0.1 & 2.6 & 5.8 & 220 & 1.6 & 1.1 & 14 & $<0.05$ & $<0.1$ \\
GR12 & 0.1 & 0.30 & 1.8 & 11 & 0.2 & 0.40 & 4.7 & $<0.05$ & 1.8 \\
GR13 & 0.2 & 0.70 & 1.7 & 130 & 0.4 & 0.40 & 3.8 & $<0.05$ & $<0.1$ \\
GR14 & 0.2 & 1.4 & 2.9 & 140 & 0.8 & 0.60 & 10 & $<0.05$ & $<0.1$ \\
GR15 & 0.5 & 3.1 & 4.1 & 130 & 1.6 & 0.84 & 9.0 & $<0.05$ & $<0.1$ \\
GR16 & $<0.1$ & 0.40 & 0.73 & 57 & 0.3 & 0.20 & 19 & $<0.05$ & $<0.1$ \\
\hline
\end{tabular}


Table 2. Chemical analyses of rocks from Guam

\begin{tabular}{cccccccccc}
\hline Field No. & $\begin{array}{c}\text { Sc } \\
\text { ppm }\end{array}$ & $\begin{array}{c}\text { Se } \\
\text { ppm }\end{array}$ & $\begin{array}{c}\text { Sm } \\
\text { ppm }\end{array}$ & $\begin{array}{c}\text { Sn } \\
\text { ppm }\end{array}$ & $\begin{array}{c}\text { Sr } \\
\text { ppm }\end{array}$ & $\begin{array}{c}\text { Tb } \\
\text { ppm }\end{array}$ & $\begin{array}{c}\text { Te } \\
\text { ppm }\end{array}$ & $\begin{array}{c}\text { Th } \\
\text { ppm }\end{array}$ & $\begin{array}{c}\text { Tl } \\
\text { ppm }\end{array}$ \\
\hline \hline LLD $^{1}$ & 0.3 & 1 & 0.02 & 0.5 & 0.05 & 0.005 & 0.1 & 0.09 & 0.1 \\
GR01 & 17 & $<1$ & 1.3 & $<0.5$ & 730 & 0.230 & $<0.1$ & 1.0 & 0.1 \\
GR02 & 4 & $<1$ & 0.75 & $<0.5$ & 870 & 0.120 & $<0.1$ & $<0.09$ & $<0.1$ \\
GR03 & 6.3 & $<1$ & 1.2 & $<0.5$ & 760 & 0.190 & $<0.1$ & $<0.09$ & $<0.1$ \\
GR04 & 6.1 & $<1$ & 0.40 & $<0.5$ & 510 & 0.089 & $<0.1$ & $<0.09$ & $<0.1$ \\
GR05 & 9 & $<1$ & 1.1 & $<0.5$ & 260 & 0.190 & $<0.1$ & 0.30 & $<0.1$ \\
GR07 & 30 & $<1$ & 2.5 & 0.6 & 260 & 0.400 & $<0.1$ & 1.3 & $<0.1$ \\
GR08 & 36 & $<1$ & 1.5 & $<0.5$ & 540 & 0.310 & $<0.1$ & 0.46 & $<0.1$ \\
GR09 & 20 & $<1$ & 1.0 & $<0.5$ & 150 & 0.210 & $<0.1$ & 0.10 & $<0.1$ \\
GR10 & 39 & $<1$ & 8.1 & 0.9 & 82 & 1.4 & $<0.1$ & 0.90 & $<0.1$ \\
GR11 & 48 & $<1$ & 1.7 & $<0.5$ & 10 & 0.390 & $<0.1$ & 0.41 & $<0.1$ \\
GR12 & 4 & $<1$ & 0.40 & $<0.5$ & 88 & 0.086 & $<0.1$ & $<0.09$ & $<0.1$ \\
GR13 & 16 & $<1$ & 0.50 & $<0.5$ & 130 & 0.130 & $<0.1$ & $<0.09$ & $<0.1$ \\
GR14 & 22 & $<1$ & 0.79 & $<0.5$ & 110 & 0.180 & $<0.1$ & 0.20 & $<0.1$ \\
GR15 & 30 & $<1$ & 1.2 & 0.6 & 150 & 0.270 & $<0.1$ & 0.47 & $<0.1$ \\
GR16 & 5.7 & $<1$ & 0.20 & $<0.5$ & 100 & 0.040 & $<0.1$ & $<0.09$ & $<0.1$ \\
\hline
\end{tabular}


Table 2. Chemical analyses of rocks from Guam

\begin{tabular}{cccccccc}
\hline Field No. & $\begin{array}{c}\text { Tm } \\
\text { ppm }\end{array}$ & $\begin{array}{c}\mathrm{U} \\
\mathrm{ppm}\end{array}$ & $\begin{array}{c}\mathrm{V} \\
\mathrm{ppm}\end{array}$ & $\begin{array}{c}\mathrm{W} \\
\mathrm{ppm}\end{array}$ & $\begin{array}{c}\mathrm{Y} \\
\mathrm{ppm}\end{array}$ & $\begin{array}{c}\text { Yb } \\
\mathrm{ppm}\end{array}$ & $\begin{array}{c}\text { Zn } \\
\mathrm{ppm}\end{array}$ \\
\hline \hline LLD $^{1}$ & 0.006 & 0.02 & 0.4 & 0.4 & 0.3 & 0.03 & 5 \\
GR01 & 0.140 & 0.40 & 100 & $<0.4$ & 14 & 1.0 & 46 \\
GR02 & 0.069 & 0.10 & 41 & $<0.4$ & 8.6 & 0.50 & 20 \\
GR03 & 0.100 & 0.20 & 42 & $<0.4$ & 12 & 0.70 & 20 \\
GR04 & 0.056 & 1.2 & 46 & $<0.4$ & 6.4 & 0.40 & 10 \\
GR05 & 0.120 & 0.30 & 55 & $<0.4$ & 12 & 0.87 & 20 \\
GR07 & 0.240 & 0.40 & 230 & $<0.4$ & 21 & 1.6 & 64 \\
GR08 & 0.200 & 0.07 & 200 & $<0.4$ & 19 & 1.4 & 64 \\
GR09 & 0.140 & 0.30 & 120 & $<0.4$ & 13 & 1.0 & 33 \\
GR10 & 0.820 & 0.82 & 340 & $<0.4$ & 83 & 5.7 & 160 \\
GR11 & 0.300 & 0.30 & 120 & $<0.4$ & 26 & 2.1 & 130 \\
GR12 & 0.083 & 0.07 & 31 & $<0.4$ & 8.1 & 0.61 & 31 \\
GR13 & 0.130 & 0.20 & 87 & $<0.4$ & 12 & 0.96 & 32 \\
GR14 & 0.140 & 0.20 & 140 & $<0.4$ & 14 & 1.0 & 41 \\
GR15 & 0.200 & 0.40 & 190 & $<0.4$ & 18 & 1.4 & 50 \\
GR16 & 0.040 & 0.20 & 39 & $<0.4$ & 3.8 & 0.30 & 10 \\
\hline
\end{tabular}

${ }^{1}$ lower limit of determination 
Table 3. Chemical analyses of streambed sediment samples from areas underlain by the Facpi Formation, Guam

\begin{tabular}{ccccccccc}
\hline Field No. & $\begin{array}{c}\text { North Latitude } \\
\text { Degree }\end{array}$ & $\begin{array}{c}\text { East Longitude } \\
\text { Degree }\end{array}$ & $\begin{array}{c}\mathrm{Al} \\
\%\end{array}$ & $\begin{array}{c}\mathrm{Ca} \\
\%\end{array}$ & $\begin{array}{c}\mathrm{Fe} \\
\%\end{array}$ & $\begin{array}{c}\mathrm{K} \\
\%\end{array}$ & $\begin{array}{c}\mathrm{Mg} \\
\%\end{array}$ & $\begin{array}{c}\mathrm{Na} \\
\%\end{array}$ \\
\hline \hline LLD $^{1}$ & & & 0.005 & 0.005 & 0.005 & 0.005 & 0.005 & 0.005 \\
GSS01 & 13.2992 & 144.6811 & 8.4 & 4.2 & 8.7 & 0.74 & 2.9 & 0.76 \\
GSS02 & 13.2992 & 144.6811 & 8.9 & 3 & 9.7 & 0.75 & 2.4 & 0.49 \\
GSS03 & 13.2967 & 144.6725 & 8.1 & 3.8 & 10 & 0.61 & 2.7 & 0.56 \\
GSS04 & 13.2783 & 144.6858 & 8.8 & 4.6 & 11 & 0.48 & 2.5 & 0.82 \\
\hline
\end{tabular}


Table 3. Chemical analyses of streambed sediment samples from areas underlain by the Facpi Formation, Guam

\begin{tabular}{cccccccccc}
\hline Field No. & $\begin{array}{c}\mathrm{P} \\
\%\end{array}$ & $\begin{array}{c}\mathrm{Ti} \\
\%\end{array}$ & $\begin{array}{c}\mathrm{Ag} \\
\mathrm{ppm}\end{array}$ & $\begin{array}{c}\mathrm{As} \\
\mathrm{ppm}\end{array}$ & $\begin{array}{c}\mathrm{Au} \\
\mathrm{ppm}\end{array}$ & $\begin{array}{c}\mathrm{Ba} \\
\mathrm{ppm}\end{array}$ & $\begin{array}{c}\mathrm{Be} \\
\mathrm{ppm}\end{array}$ & $\begin{array}{c}\mathrm{Bi} \\
\mathrm{ppm}\end{array}$ & $\begin{array}{c}\mathrm{Cd} \\
\mathrm{ppm}\end{array}$ \\
\hline \hline LLD $^{1}$ & 0.01 & 0.005 & 3 & 0.5 & 0.05 & 0.5 & 0.1 & 0.05 & 0.1 \\
GSS01 & 0.06 & 0.80 & $<3$ & 0.6 & $<0.05$ & 210 & 0.5 & $<0.05$ & 0.2 \\
GSS02 & 0.07 & 1.0 & $<3$ & 0.6 & $<0.05$ & 220 & 0.6 & $<0.05$ & 0.2 \\
GSS03 & 0.04 & 1.0 & $<3$ & 2.0 & $<0.05$ & 280 & 0.5 & $<0.05$ & 0.2 \\
GSS04 & 0.07 & 1.1 & $<3$ & $<0.5$ & $<0.05$ & 180 & 0.5 & $<0.05$ & 0.1 \\
\hline
\end{tabular}


Table 3. Chemical analyses of streambed sediment samples from areas underlain by the Facpi Formation, Guam

\begin{tabular}{cccccccccc}
\hline Field No. & $\begin{array}{c}\mathrm{Ce} \\
\mathrm{ppm}\end{array}$ & $\begin{array}{c}\mathrm{Co} \\
\mathrm{ppm}\end{array}$ & $\begin{array}{c}\mathrm{Cr} \\
\mathrm{ppm}\end{array}$ & $\begin{array}{c}\mathrm{Cs} \\
\mathrm{ppm}\end{array}$ & $\begin{array}{c}\mathrm{Cu} \\
\mathrm{ppm}\end{array}$ & $\begin{array}{c}\mathrm{Dy} \\
\mathrm{ppm}\end{array}$ & $\begin{array}{c}\mathrm{Er} \\
\mathrm{ppm}\end{array}$ & $\begin{array}{c}\mathrm{Eu} \\
\mathrm{ppm}\end{array}$ & $\begin{array}{c}\mathrm{Ga} \\
\mathrm{ppm}\end{array}$ \\
\hline \hline LLD $^{1}$ & 0.5 & 0.1 & 0.2 & 0.01 & 3 & 0.04 & 0.05 & 0.005 & 0.006 \\
GSS01 & 14 & 38 & 68 & 0.40 & 130 & 2.7 & 1.5 & 0.71 & 16 \\
GSS02 & 19 & 42 & 53 & 0.40 & 160 & 3.5 & 2.0 & 0.88 & 18 \\
GSS03 & 12 & 46 & 180 & 0.40 & 130 & 2.9 & 1.6 & 0.73 & 17 \\
GSS04 & 18 & 46 & 91 & 0.40 & 150 & 3.4 & 1.9 & 0.92 & 18 \\
\hline
\end{tabular}


Table 3. Chemical analyses of streambed sediment samples from areas underlain by the Facpi Formation, Guam

\begin{tabular}{cccccccccc}
\hline Field No. & $\begin{array}{c}\text { Gd } \\
\text { ppm }\end{array}$ & $\begin{array}{c}\text { Ge } \\
\text { ppm }\end{array}$ & $\begin{array}{c}\text { Ho } \\
\text { ppm }\end{array}$ & $\begin{array}{c}\text { In } \\
\text { ppm }\end{array}$ & $\begin{array}{c}\text { La } \\
\text { ppm }\end{array}$ & $\begin{array}{c}\text { Li } \\
\text { ppm }\end{array}$ & $\begin{array}{c}\text { Mn } \\
\text { ppm }\end{array}$ & $\begin{array}{c}\text { Mo } \\
\text { ppm }\end{array}$ & $\begin{array}{c}\text { Nb } \\
\text { ppm }\end{array}$ \\
\hline \hline LLD $^{1}$ & 0.02 & 0.1 & 0.02 & 0.1 & 0.3 & 0.2 & 0.2 & 0.1 & 2 \\
GSS01 & 2.3 & 1.2 & 0.46 & $<0.1$ & 6.5 & 20 & 1200 & 0.3 & 2.4 \\
GSS02 & 3.2 & 1.4 & 0.61 & $<0.1$ & 9.0 & 20 & 1700 & 0.3 & 3.2 \\
GSS03 & 2.6 & 1.3 & 0.50 & $<0.1$ & 7.6 & 31 & 1600 & 0.3 & 2.3 \\
GSS04 & 3.1 & 1.4 & 0.59 & $<0.1$ & 8.4 & 17 & 1700 & 0.3 & 3.3 \\
\hline
\end{tabular}


Table 3. Chemical analyses of streambed sediment samples from areas underlain by the Facpi Formation, Guam

\begin{tabular}{cccccccccc}
\hline Field No. & $\begin{array}{c}\mathrm{Nd} \\
\mathrm{ppm}\end{array}$ & $\begin{array}{c}\mathrm{Ni} \\
\mathrm{ppm}\end{array}$ & $\begin{array}{c}\mathrm{Pb} \\
\mathrm{ppm}\end{array}$ & $\begin{array}{c}\mathrm{Pr} \\
\mathrm{ppm}\end{array}$ & $\begin{array}{c}\mathrm{Rb} \\
\mathrm{ppm}\end{array}$ & $\begin{array}{c}\mathrm{Re} \\
\mathrm{ppm}\end{array}$ & $\begin{array}{c}\mathrm{Sb} \\
\mathrm{ppm}\end{array}$ & $\begin{array}{c}\mathrm{Sc} \\
\mathrm{ppm}\end{array}$ & $\begin{array}{c}\mathrm{Se} \\
\mathrm{ppm}\end{array}$ \\
\hline \hline LLD $^{1}$ & 0.2 & 1 & 0.8 & 0.05 & 0.01 & 0.05 & 0.1 & 0.3 & 1 \\
GSS01 & 8.6 & 28 & 3.4 & 1.8 & 19 & $<0.05$ & 2.0 & 34 & $<1$ \\
GSS02 & 12 & 25 & 3.8 & 2.4 & 20 & $<0.05$ & 1.5 & 34 & $<1$ \\
GSS03 & 9.5 & 72 & 3.4 & 1.9 & 16 & $<0.05$ & 0.5 & 36 & $<1$ \\
GSS04 & 12 & 30 & 3.0 & 2.3 & 16 & $<0.05$ & 0.3 & 41 & $<1$ \\
\hline
\end{tabular}


Table 3. Chemical analyses of streambed sediment samples from areas underlain by the Facpi Formation, Guam

\begin{tabular}{cccccccccc}
\hline Field No. & $\begin{array}{c}\text { Sm } \\
\text { ppm }\end{array}$ & $\begin{array}{c}\text { Sn } \\
\text { ppm }\end{array}$ & $\begin{array}{c}\text { Sr } \\
\text { ppm }\end{array}$ & $\begin{array}{c}\text { Tb } \\
\text { ppm }\end{array}$ & $\begin{array}{c}\text { Te } \\
\text { ppm }\end{array}$ & $\begin{array}{c}\text { Th } \\
\text { ppm }\end{array}$ & $\begin{array}{c}\text { Tl } \\
\text { ppm }\end{array}$ & $\begin{array}{c}\text { Tm } \\
\text { ppm }\end{array}$ & $\begin{array}{c}\text { U } \\
\text { ppm }\end{array}$ \\
\hline \hline LLD $^{1}$ & 0.02 & 0.5 & 0.05 & 0.005 & 0.1 & 0.03 & 0.1 & 0.006 & 0.02 \\
GSS01 & 2.1 & 0.9 & 260 & 0.35 & $<0.1$ & 0.61 & $<0.1$ & 0.22 & 0.40 \\
GSS02 & 2.9 & 1.0 & 200 & 0.47 & $<0.1$ & 0.9 & $<0.1$ & 0.29 & 0.52 \\
GSS03 & 2.2 & 0.9 & 280 & 0.38 & $<0.1$ & 0.55 & $<0.1$ & 0.23 & 0.40 \\
GSS04 & 2.8 & 1.0 & 270 & 0.45 & $<0.1$ & 0.78 & $<0.1$ & 0.27 & 0.40 \\
\hline
\end{tabular}


Table 3. Chemical analyses of streambed sediment samples from areas underlain by the Facpi Formation, Guam

\begin{tabular}{cccccc}
\hline Field No. & $\begin{array}{c}\text { V } \\
\text { ppm }\end{array}$ & $\begin{array}{c}\text { W } \\
\text { ppm }\end{array}$ & $\begin{array}{c}\text { Y } \\
\text { ppm }\end{array}$ & $\begin{array}{c}\text { Yb } \\
\text { ppm }\end{array}$ & $\begin{array}{c}\text { Zn } \\
\text { ppm }\end{array}$ \\
\hline \hline LLD $^{1}$ & 0.4 & 0.1 & 0.3 & 0.03 & 5 \\
GSS01 & 340 & 0.1 & 20 & 1.5 & 96 \\
GSS02 & 430 & 0.2 & 27 & 2.0 & 110 \\
GSS03 & 450 & 0.2 & 21 & 1.6 & 120 \\
GSS04 & 490 & 0.2 & 25 & 1.8 & 110 \\
\hline
\end{tabular}


Table 4. Chemical analyses of soil samples from the western United States

\begin{tabular}{|c|c|c|c|c|c|c|c|}
\hline Field No. & Latitude & Longitude & Rock Type & $\mathrm{Al}$ & $\mathrm{Ca}$ & $\mathrm{Fe}$ & $\mathrm{K}$ \\
\hline & Degree & Degree & & $\%$ & $\%$ & $\%$ & $\%$ \\
\hline $\operatorname{LLD}^{1}$ & & & & 0.005 & 0.005 & 0.005 & 0.005 \\
\hline CS01 & 38.5067 & 106.6623 & Tuffaceous sediments & 6.7 & 2.3 & 3.2 & 2.0 \\
\hline CS02 & 38.5075 & 106.6638 & Tuffaceous sediments & 6.3 & 3.0 & 3.0 & 2.0 \\
\hline CS03 & 38.6146 & 106.772 & Tuffaceous sediments & 7.2 & 0.77 & 3.4 & 2.2 \\
\hline CS04 & 38.4050 & 107.0766 & Ash flow tuff & 7.3 & 1.5 & 3.9 & 1.9 \\
\hline CS05 & 38.4159 & 107.0763 & Ash flow tuff & 2.9 & 17 & 1.3 & 0.94 \\
\hline CS06 & 37.9266 & 107.4576 & Mineralized tuff breccia & 6.2 & 0.40 & 2.7 & 3.3 \\
\hline CS07 & 37.9162 & 107.4455 & Mineralized tuff breccia & 5.9 & 0.60 & 2.5 & 2.7 \\
\hline CS08 & 38.5590 & 107.3282 & Andesite & 7.4 & 1.7 & 3.4 & 2.2 \\
\hline CS09 & 38.5452 & 107.3196 & Andesite & 6.6 & 1.2 & 3.3 & 2.2 \\
\hline GS10 & 38.4939 & 107.7343 & Marine shale & 5.6 & 4.0 & 2.4 & 1.8 \\
\hline CS11 & 38.5312 & 107.937 & Marine shale & 5.1 & 14 & 2.3 & 1.6 \\
\hline CS12 & 39.0315 & 108.0592 & Basalt & 6.5 & 1.1 & 3.8 & 1.7 \\
\hline CS13 & 39.0097 & 108.1044 & Basalt & 7.5 & 0.80 & 4.4 & 1.6 \\
\hline CS14 & 39.6566 & 106.9558 & Evaporite & 3.5 & 9.7 & 1.7 & 1.6 \\
\hline CS15 & 39.6643 & 106.9513 & Evaporite & 2.3 & 11 & 0.97 & 1.5 \\
\hline CS16 & 39.5188 & 106.3526 & Clastic sedimentary rock & 8.9 & 1.3 & 3.6 & 3.4 \\
\hline CS17 & 39.5556 & 106.272 & Clastic sedimentary rock & 6.9 & 0.50 & 3.7 & 2.5 \\
\hline CS18 & 39.7511 & 105.174 & Basalt & 7.4 & 1.6 & 4.4 & 3.1 \\
\hline CS19 & 39.7511 & 105.1624 & Basalt & 8.2 & 1.7 & 5.2 & 3.4 \\
\hline S01 & 38.0568 & 106.1420 & Andesite & 8.4 & 2.4 & 6.9 & 0.53 \\
\hline S02 & 37.8108 & 106.2868 & Ash flow tuff & 9.6 & 2.5 & 8.3 & 0.82 \\
\hline S03 & 37.7921 & 106.3230 & Andesite & 8.5 & 1.2 & 7.2 & 0.52 \\
\hline S04 & 37.7537 & 106.3084 & Ash flow tuff & 8.0 & 1.3 & 6.8 & 0.27 \\
\hline S05 & 37.7485 & 106.2910 & Ash flow tuff & 9.2 & 1.9 & 7.8 & 0.37 \\
\hline S06 & 37.7418 & 106.3537 & Andesite & 8.4 & 1.6 & 8.2 & 0.26 \\
\hline S07 & 37.7329 & 106.3524 & Dacite and rhyodacite & 9.8 & 1.2 & 8.6 & 0.19 \\
\hline S08 & 37.2584 & 106.1360 & Basalt & 8.5 & 11 & 6.1 & 0.44 \\
\hline S09 & 37.2484 & 106.1443 & Basalt & 15 & $<0.05$ & 12 & 0.12 \\
\hline S10 & 37.2631 & 105.8350 & Andesite & 10 & 1.5 & 8.0 & 0.87 \\
\hline S11 & 37.1900 & 105.8049 & Andesite & 9.0 & 1.6 & 6.3 & 1.2 \\
\hline S12 & 37.1124 & 105.8651 & Andesite & 8.3 & 2.1 & 6.5 & 1.0 \\
\hline S13 & 36.9062 & 105.9887 & Dacite and rhyodacite & 8.0 & 4.9 & 7.2 & 0.30 \\
\hline S14 & 36.9045 & 105.9892 & Dacite and rhyodacite & 6.1 & 6.0 & 4.5 & 0.71 \\
\hline S15 & 36.8518 & 106.0727 & Basalt & 7.7 & 2.0 & 6.2 & 0.64 \\
\hline S16 & 36.8261 & 106.0615 & Basalt & 8.7 & 2.4 & 7.3 & 0.65 \\
\hline $\mathrm{S} 17$ & 36.8101 & 106.0254 & Andesite & 6.6 & 3.4 & 6.5 & 0.42 \\
\hline
\end{tabular}

${ }^{1}$ lower limit of determination 
Table 4. Chemical analyses of soil samples from the western United States

\begin{tabular}{|c|c|c|c|c|c|c|c|c|c|}
\hline Field No. & $\mathrm{Mg}$ & $\mathrm{Na}$ & $\mathrm{P}$ & $\mathrm{Ti}$ & $\mathrm{Ag}$ & As & $\mathrm{Au}$ & $\mathrm{Ba}$ & $\mathrm{Be}$ \\
\hline & $\%$ & $\%$ & $\%$ & $\%$ & ppm & ppm & $\mathrm{ppm}$ & ppm & $\mathrm{ppm}$ \\
\hline $\operatorname{LLD}^{1}$ & 0.005 & 0.005 & 0.01 & 0.005 & 3 & 0.5 & 0.05 & 0.5 & 0.1 \\
\hline CS01 & 1.2 & 1.1 & 0.12 & 0.50 & $<3$ & 4.0 & $<0.05$ & 690 & 3.1 \\
\hline CS02 & 1.1 & 1.2 & 0.11 & 0.40 & $<3$ & 4.0 & $<0.05$ & 740 & 3.2 \\
\hline CS03 & 0.74 & 0.58 & 0.08 & 0.40 & $<3$ & 6.4 & $<0.05$ & 650 & 2.8 \\
\hline CS04 & 1.3 & 1.2 & 0.07 & 0.50 & $<3$ & 4.0 & $<0.05$ & 640 & 2.5 \\
\hline CS05 & 1.3 & 0.48 & 0.05 & 0.20 & $<3$ & 6.2 & $<0.05$ & 760 & 1.0 \\
\hline CS06 & 0.53 & 0.71 & 0.07 & 0.20 & $<3$ & 38 & $<0.05$ & 410 & 5.1 \\
\hline CS07 & 0.59 & 1.1 & 0.06 & 0.30 & $<3$ & 9.6 & $<0.05$ & 570 & 2.7 \\
\hline CS08 & 0.87 & 0.88 & 0.13 & 0.40 & $<3$ & 6.1 & $<0.05$ & 630 & 2.1 \\
\hline CS09 & 0.72 & 0.79 & 0.10 & 0.40 & $<3$ & 6.0 & $<0.05$ & 680 & 1.7 \\
\hline GS10 & 2.0 & 0.42 & 0.09 & 0.30 & $<3$ & 8.4 & $<0.05$ & 430 & 1.7 \\
\hline CS11 & 1.3 & 0.26 & 0.09 & 0.30 & $<3$ & 9.8 & $<0.05$ & 520 & 1.5 \\
\hline CS12 & 1.2 & 0.93 & 0.08 & 0.60 & $<3$ & 5.1 & $<0.05$ & 670 & 1.4 \\
\hline CS13 & 1.0 & 0.78 & 0.12 & 0.70 & $<3$ & 5.0 & $<0.05$ & 720 & 1.5 \\
\hline CS14 & 2.0 & 0.79 & 0.05 & 0.20 & $<3$ & 2.0 & $<0.05$ & 310 & 1.1 \\
\hline CS15 & 3.2 & 0.27 & 0.03 & 0.09 & $<3$ & 2.0 & $<0.05$ & 240 & 0.80 \\
\hline CS16 & 1.3 & 0.31 & 0.05 & 0.40 & $<3$ & 2.0 & $<0.05$ & 940 & 3.9 \\
\hline CS17 & 0.92 & 1.2 & 0.11 & 0.30 & $<3$ & 1.0 & $<0.05$ & 570 & 3.1 \\
\hline CS18 & 0.76 & 1.6 & 0.16 & 0.50 & $<3$ & 5.7 & $<0.05$ & 1000 & 1.9 \\
\hline CS19 & 0.90 & 1.5 & 0.20 & 0.60 & $<3$ & 8.6 & $<0.05$ & 970 & 2.7 \\
\hline S01 & 1.8 & 0.55 & 0.06 & 0.70 & $<3$ & 1.0 & $<0.05$ & 320 & 0.50 \\
\hline S02 & 2.2 & 0.40 & 0.04 & 0.80 & $<3$ & 1.0 & $<0.05$ & 590 & 0.60 \\
\hline S03 & 1.6 & 0.31 & 0.05 & 0.80 & $<3$ & 2.0 & $<0.05$ & 240 & 1.6 \\
\hline S04 & 1.4 & 0.05 & 0.07 & 0.50 & $<3$ & 6.1 & $<0.05$ & 240 & 0.70 \\
\hline S05 & 1.1 & 0.14 & 0.12 & 0.70 & $<3$ & 3.0 & $<0.05$ & 120 & 1.90 \\
\hline S06 & 1.1 & 0.17 & 0.10 & 0.80 & $<3$ & 2.0 & $<0.05$ & 250 & 0.40 \\
\hline S07 & 1.2 & 0.03 & 0.04 & 0.80 & $<3$ & 1.0 & $<0.05$ & 53 & 0.80 \\
\hline S08 & 1.4 & 0.24 & 0.07 & 0.60 & $<3$ & 2.0 & $<0.05$ & 170 & 0.40 \\
\hline S09 & 0.61 & 0.03 & 0.03 & 1.0 & $<3$ & $<0.5$ & $<0.05$ & 72 & 1.0 \\
\hline $\mathrm{S} 10$ & 2.1 & 0.63 & 0.02 & 0.70 & $<3$ & $<0.5$ & $<0.05$ & 680 & 0.70 \\
\hline S11 & 1.8 & 0.64 & 0.07 & 0.70 & $<3$ & $<0.5$ & $<0.05$ & 740 & 1.5 \\
\hline S12 & 3.4 & 0.08 & 0.02 & 0.40 & $<3$ & 3.0 & $<0.05$ & 540 & 0.60 \\
\hline S13 & 3.9 & 0.42 & 0.02 & 0.40 & $<3$ & 2.0 & $<0.05$ & 19 & 0.30 \\
\hline S14 & 2.7 & 0.18 & 0.04 & 0.30 & $<3$ & $<0.5$ & 0.09 & 300 & 0.60 \\
\hline S15 & 6.9 & 0.29 & 0.01 & 0.30 & $<3$ & $<0.5$ & $<0.05$ & 46 & 0.40 \\
\hline S16 & 5.3 & 0.28 & $<0.01$ & 0.40 & $<3$ & $<0.5$ & $<0.05$ & 61 & 0.40 \\
\hline S17 & 7.4 & 0.46 & 0.02 & 0.30 & $<3$ & $<0.5$ & $<0.05$ & 24 & 0.40 \\
\hline
\end{tabular}


Table 4. Chemical analyses of soil samples from the western United States

\begin{tabular}{|c|c|c|c|c|c|c|c|c|c|}
\hline Field No. & $\mathrm{Bi}$ & $\mathrm{Cd}$ & $\mathrm{Ce}$ & $\mathrm{Co}$ & $\mathrm{Cr}$ & Cs & $\mathrm{Cu}$ & Dy & $\mathrm{Er}$ \\
\hline & $\mathrm{ppm}$ & $\mathrm{ppm}$ & $\mathrm{ppm}$ & ppm & ppm & $\mathrm{ppm}$ & ppm & $\mathrm{ppm}$ & $\mathrm{ppm}$ \\
\hline $\operatorname{LLD}^{1}$ & 0.05 & 0.1 & 0.5 & 0.1 & 0.2 & 0.01 & 3 & 0.04 & 0.05 \\
\hline CS01 & 0.40 & 0.2 & 95 & 11 & 42 & 7.2 & 20 & 7.5 & 4.2 \\
\hline CS02 & 0.50 & 0.3 & 72 & 10 & 33 & 8.8 & 30 & 5.5 & 3.2 \\
\hline CS03 & 0.30 & 0.6 & 100 & 14 & 56 & 7.9 & 30 & 5.9 & 2.6 \\
\hline CS04 & 0.20 & 0.1 & 83 & 11 & 35 & 6.9 & 20 & 6.3 & 3.8 \\
\hline CS05 & 0.07 & 0.2 & 39 & 3.1 & 17 & 2.6 & 9 & 3.3 & 1.9 \\
\hline CS06 & 0.30 & 0.3 & 160 & 7.4 & 15 & 24 & 20 & 5.7 & 3.1 \\
\hline CS07 & 0.50 & 0.3 & 120 & 8.9 & 24 & 12 & 30 & 5.7 & 3.0 \\
\hline CS08 & 0.30 & 1.3 & 57 & 8.1 & 49 & 7.6 & 30 & 5.3 & 3.1 \\
\hline CS09 & 0.20 & 0.9 & 69 & 11 & 43 & 6.4 & 30 & 5.6 & 3.2 \\
\hline GS10 & 0.20 & 0.3 & 56 & 7.2 & 63 & 9.2 & 20 & 4.6 & 2.6 \\
\hline CS11 & 0.20 & 0.9 & 45 & 9.4 & 65 & 7.4 & 20 & 4.1 & 2.4 \\
\hline CS12 & 0.20 & 0.2 & 61 & 17 & 110 & 4.2 & 30 & 4.0 & 2.2 \\
\hline CS13 & 0.20 & 0.2 & 67 & 20 & 99 & 4.5 & 30 & 3.9 & 2.1 \\
\hline CS14 & 0.06 & 0.2 & 40 & 6.6 & 41 & 2.3 & 10 & 2.9 & 1.6 \\
\hline CS15 & $<0.05$ & $<0.1$ & 24 & 4.0 & 32 & 1.3 & 8 & 2.3 & 1.3 \\
\hline CS16 & 0.40 & 0.2 & 68 & 12 & 96 & 7.8 & 9 & 4.3 & 2.1 \\
\hline CS17 & 0.30 & 0.4 & 220 & 12 & 75 & 6.1 & 20 & 10 & 4.2 \\
\hline CS18 & 0.50 & 0.9 & 120 & 15 & 48 & 4.3 & 44 & 7.1 & 3.6 \\
\hline CS19 & 0.59 & 1.5 & 100 & 18 & 45 & 4.7 & 66 & 6.7 & 3.7 \\
\hline S01 & $<0.05$ & 0.3 & 17 & 31 & 97 & 0.40 & 140 & 3.9 & 2.2 \\
\hline S02 & $<0.05$ & 0.2 & 17 & 38 & 45 & 0.40 & 140 & 4.4 & 2.6 \\
\hline S03 & 0.07 & 0.5 & 29 & 33 & 70 & 0.60 & 160 & 6.7 & 3.8 \\
\hline S04 & $<0.05$ & 0.3 & 12 & 58 & 270 & 0.50 & 130 & 4.0 & 2.3 \\
\hline S05 & $<0.05$ & 1.6 & 61 & 44 & 110 & 0.70 & 140 & 22 & 13 \\
\hline S06 & $<0.05$ & 0.8 & 31 & 53 & 27 & 0.50 & 120 & 5.9 & 3.5 \\
\hline S07 & $<0.05$ & 0.5 & 26 & 40 & 62 & 0.60 & 130 & 6.1 & 3.6 \\
\hline S08 & $<0.05$ & 0.4 & 15 & 23 & 70 & 0.50 & 60 & 4.4 & 2.6 \\
\hline S09 & $<0.05$ & 0.1 & 44 & 160 & 40 & 0.90 & 280 & 5.7 & 3.2 \\
\hline $\mathrm{S} 10$ & $<0.05$ & 0.1 & 41 & 40 & 60 & 0.60 & 130 & 6.8 & 3.8 \\
\hline S11 & $<0.05$ & 0.3 & 57 & 31 & 21 & 0.40 & 110 & 7.2 & 4.0 \\
\hline S12 & $<0.05$ & 0.3 & 11 & 49 & 450 & 0.50 & 96 & 3.1 & 1.8 \\
\hline S13 & $<0.05$ & 0.2 & 4.6 & 50 & 540 & 0.20 & 89 & 2.8 & 1.8 \\
\hline S14 & $<0.05$ & 0.3 & 7.1 & 27 & 190 & 0.30 & 93 & 4.4 & 2.8 \\
\hline S15 & $<0.05$ & 0.1 & 6.9 & 53 & 580 & 0.30 & 67 & 2.4 & 1.5 \\
\hline S16 & $<0.05$ & 0.2 & 5.3 & 50 & 670 & 0.20 & 79 & 2.6 & 1.7 \\
\hline S17 & $<0.05$ & $<0.1$ & 6.7 & 51 & 530 & 0.10 & 77 & 2.3 & 1.4 \\
\hline
\end{tabular}


Table 4. Chemical analyses of soil samples from the western United States

\begin{tabular}{|c|c|c|c|c|c|c|c|c|c|}
\hline Field No. & $\mathrm{Eu}$ & $\mathrm{Ga}$ & $\mathrm{Gd}$ & $\mathrm{Ge}$ & Ho & In & $\mathrm{La}$ & $\mathrm{Li}$ & $\mathrm{Mn}$ \\
\hline & ppm & ppm & ppm & ppm & ppm & $\mathrm{ppm}$ & $\mathrm{ppm}$ & $\mathrm{ppm}$ & $\mathrm{ppm}$ \\
\hline LLD $^{1}$ & 0.005 & 0.006 & 0.02 & 0.1 & 0.02 & 0.1 & 0.3 & 0.2 & 0.2 \\
\hline CS01 & 1.5 & 17 & 7.1 & 1.1 & 1.5 & $<0.1$ & 51 & 40 & 630 \\
\hline $\mathrm{CS} 02$ & 1.4 & 15 & 5.4 & 1.0 & 1.1 & $<0.1$ & 40 & 41 & 870 \\
\hline $\mathrm{CS} 03$ & 1.8 & 18 & 7.0 & 1.1 & 1.1 & $<0.1$ & 59 & 51 & 730 \\
\hline CS04 & 1.6 & 18 & 6.4 & 1.3 & 1.3 & $<0.1$ & 48 & 57 & 590 \\
\hline CS05 & 0.68 & 7.4 & 3.0 & 0.5 & 0.69 & $<0.1$ & 22 & 17 & 180 \\
\hline CS06 & 0.97 & 18 & 5.5 & 1.0 & 1.2 & $<0.1$ & 64 & 35 & 1200 \\
\hline $\mathrm{CS} 07$ & 1.6 & 14 & 6.0 & 1.0 & 1.1 & $<0.1$ & 56 & 32 & 1100 \\
\hline CS08 & 1.5 & 18 & 5.1 & 1.2 & 1.1 & $<0.1$ & 33 & 30 & 460 \\
\hline CS09 & 1.6 & 16 & 5.6 & 1.1 & 1.1 & $<0.1$ & 38 & 34 & 770 \\
\hline GS10 & 1.2 & 14 & 4.5 & 0.8 & 0.95 & $<0.1$ & 31 & 41 & 180 \\
\hline CS11 & 0.96 & 13 & 3.8 & 0.8 & 0.86 & $<0.1$ & 28 & 33 & 210 \\
\hline CS12 & 1.1 & 16 & 4.0 & 1.2 & 0.83 & $<0.1$ & 30 & 28 & 610 \\
\hline CS13 & 1.2 & 18 & 4.0 & 1.2 & 0.77 & $<0.1$ & 30 & 30 & 760 \\
\hline CS14 & 0.73 & 8.6 & 2.9 & 0.6 & 0.58 & $<0.1$ & 20 & 32 & 280 \\
\hline CS15 & 0.5 & 5.3 & 2.1 & 0.5 & 0.47 & $<0.1$ & 14 & 29 & 180 \\
\hline CS16 & 1.3 & 24 & 5.1 & 1.1 & 0.82 & 0.1 & 37 & 45 & 650 \\
\hline CS17 & 2.4 & 18 & 14 & 1.1 & 1.8 & $<0.1$ & 110 & 39 & 1200 \\
\hline CS18 & 2.0 & 18 & 7.9 & 1.1 & 1.4 & 0.1 & 66 & 20 & 920 \\
\hline CS19 & 2.0 & 20 & 7.2 & 1.1 & 1.4 & 0.1 & 57 & 23 & 1200 \\
\hline S01 & 0.99 & 15 & 3.5 & 1.2 & 0.66 & $<0.1$ & 11 & 25 & 860 \\
\hline S02 & 1.0 & 17 & 3.8 & 1.7 & 0.77 & $<0.1$ & 9.4 & 21 & 1300 \\
\hline $\mathrm{S} 03$ & 1.7 & 16 & 6.2 & 1.1 & 1.1 & $<0.1$ & 20 & 33 & 2800 \\
\hline S04 & 0.82 & 14 & 3.5 & 1.9 & 0.69 & $<0.1$ & 11 & 26 & 1500 \\
\hline S05 & 4.9 & 16 & 18 & 1.2 & 3.8 & $<0.1$ & 29 & 36 & 2500 \\
\hline S06 & 1.2 & 16 & 4.8 & 1.3 & 1.0 & $<0.1$ & 11 & 18 & 5100 \\
\hline S07 & 1.2 & 18 & 5.0 & 1.5 & 1.1 & $<0.1$ & 9.8 & 19 & 1900 \\
\hline S08 & 1.0 & 14 & 4.0 & 1.0 & 0.78 & $<0.1$ & 9.2 & 33 & 1300 \\
\hline S09 & 1.4 & 27 & 5.3 & 1.8 & 0.97 & $<0.1$ & 18 & 8.2 & 3200 \\
\hline S10 & 1.8 & 15 & 6.6 & 1.2 & 1.2 & $<0.1$ & 20 & 17 & 1500 \\
\hline S11 & 1.8 & 15 & 7.3 & 0.9 & 1.2 & $<0.1$ & 30 & 16 & 1300 \\
\hline S12 & 0.65 & 14 & 2.6 & 1.2 & 0.54 & $<0.1$ & 7.3 & 46 & 1400 \\
\hline S13 & 0.52 & 14 & 2.1 & 1.2 & 0.53 & $<0.1$ & 2.6 & 30 & 1200 \\
\hline S14 & 0.83 & 9.7 & 3.7 & 0.7 & 0.82 & $<0.1$ & 9.9 & 19 & 1200 \\
\hline S15 & 0.44 & 12 & 1.8 & 1.0 & 0.43 & $<0.1$ & 3.3 & 26 & 1200 \\
\hline S16 & 0.49 & 13 & 2.0 & 1.5 & 0.50 & $<0.1$ & 4.0 & 37 & 1200 \\
\hline S17 & 0.45 & 12 & 1.8 & 1.1 & 0.42 & $<0.1$ & 3.8 & 28 & 1200 \\
\hline
\end{tabular}


Table 4. Chemical analyses of soil samples from the western United States

\begin{tabular}{|c|c|c|c|c|c|c|c|c|c|}
\hline Field No. & Mo & $\mathrm{Nb}$ & $\mathrm{Nd}$ & $\mathrm{Ni}$ & $\mathrm{Pb}$ & $\mathrm{Pr}$ & $\mathrm{Rb}$ & $\operatorname{Re}$ & $\mathrm{Sb}$ \\
\hline & $\mathrm{ppm}$ & ppm & ppm & ppm & ppm & ppm & $\mathrm{ppm}$ & $\mathrm{ppm}$ & $\mathrm{ppm}$ \\
\hline LLD $^{1}$ & 0.1 & 1 & 0.2 & 1 & 0.8 & 0.05 & 0.01 & 0.05 & 0.1 \\
\hline CS01 & 0.8 & 24 & 42 & 21 & 24 & 11 & 96 & $<0.05$ & 1.0 \\
\hline $\mathrm{CS} 02$ & 1.0 & 18 & 33 & 18 & 27 & 8.5 & 93 & $<0.05$ & 0.9 \\
\hline $\mathrm{CS} 03$ & 1.8 & 16 & 49 & 29 & 26 & 13 & 130 & $<0.05$ & 0.7 \\
\hline CS04 & 1.0 & 20 & 40 & 23 & 26 & 10 & 90 & $<0.05$ & 0.8 \\
\hline CS05 & 0.7 & 7.9 & 18 & 10 & 10 & 4.8 & 44 & $<0.05$ & 0.8 \\
\hline CS06 & 12 & 46 & 39 & 6.6 & 56 & 11 & 260 & $<0.05$ & 2.2 \\
\hline $\mathrm{CS} 07$ & 1.8 & 25 & 40 & 10 & 36 & 11 & 160 & $<0.05$ & 1.2 \\
\hline CS08 & 1.8 & 13 & 30 & 18 & 22 & 7.4 & 140 & $<0.05$ & 0.9 \\
\hline CS09 & 1.0 & 14 & 34 & 20 & 21 & 8.5 & 110 & $<0.05$ & 1.0 \\
\hline GS10 & 1.8 & 10 & 27 & 23 & 20 & 6.8 & 93 & $<0.05$ & 1.0 \\
\hline CS11 & 7.1 & 12 & 23 & 36 & 16 & 5.8 & 93 & $<0.05$ & 1.1 \\
\hline CS12 & 1.1 & 16 & 26 & 47 & 26 & 6.6 & 76 & $<0.05$ & 0.6 \\
\hline CS13 & 1.1 & 16 & 25 & 48 & 17 & 6.4 & 81 & $<0.05$ & 0.6 \\
\hline CS14 & 2.8 & 5.8 & 18 & 18 & 7.7 & 4.7 & 58 & $<0.05$ & 0.3 \\
\hline CS15 & 9.5 & 2.8 & 12 & 13 & 4.5 & 3.1 & 40 & $<0.05$ & 0.3 \\
\hline CS16 & 0.4 & 16 & 32 & 32 & 17 & 8.8 & 160 & $<0.05$ & 0.2 \\
\hline CS17 & 0.7 & 16 & 100 & 26 & 24 & 27 & 160 & $<0.05$ & 0.3 \\
\hline CS18 & 2.3 & 24 & 57 & 15 & 69 & 15 & 130 & $<0.05$ & 0.9 \\
\hline CS19 & 2.4 & 29 & 49 & 16 & 97 & 12 & 150 & $<0.05$ & 1.1 \\
\hline S01 & 0.9 & 2.9 & 14 & 57 & 7.6 & 2.7 & 17 & $<0.05$ & 0.4 \\
\hline S02 & 0.4 & 3.1 & 13 & 29 & 4.2 & 2.6 & 24 & $<0.05$ & 0.3 \\
\hline $\mathrm{S} 03$ & 0.5 & 10 & 26 & 37 & 6.9 & 5.2 & 16 & $<0.05$ & 0.3 \\
\hline S04 & 3.2 & 1.7 & 12 & 160 & 11 & 2.5 & 12 & $<0.05$ & 0.5 \\
\hline S05 & 0.8 & 3.8 & 54 & 54 & 8.8 & 10 & 19 & $<0.05$ & 0.3 \\
\hline S06 & 0.9 & 2.7 & 17 & 21 & 41 & 3.3 & 13 & $<0.05$ & 0.5 \\
\hline S07 & 0.5 & 3.2 & 15 & 32 & 5.7 & 3.0 & 10 & $<0.05$ & 0.4 \\
\hline S08 & 0.2 & 2.9 & 13 & 38 & 2.0 & 2.5 & 14 & $<0.05$ & 0.3 \\
\hline S09 & $<0.1$ & 7.1 & 21 & 35 & 4.7 & 4.4 & 6.6 & $<0.05$ & 0.2 \\
\hline S10 & 0.2 & 3.2 & 27 & 28 & 4.0 & 5.7 & 40 & $<0.05$ & 0.1 \\
\hline S11 & 0.4 & 7.2 & 34 & 14 & 7.2 & 7.2 & 34 & $<0.05$ & 0.4 \\
\hline S12 & 0.2 & 2.6 & 8.7 & 230 & 5.1 & 1.8 & 25 & $<0.05$ & 0.5 \\
\hline S13 & 0.4 & 1.4 & 4.6 & 210 & 4.2 & 0.83 & 8.0 & $<0.05$ & 0.3 \\
\hline S14 & 0.3 & 1.0 & 12 & 89 & 6.8 & 2.3 & 16 & $<0.05$ & 0.2 \\
\hline S15 & 0.1 & 1.7 & 4.7 & 320 & 1.0 & 0.92 & 18 & $<0.05$ & $<0.1$ \\
\hline S16 & 0.3 & 2.0 & 5.0 & 410 & 1.0 & 0.98 & 15 & $<0.05$ & 0.2 \\
\hline S17 & 0.2 & 1.6 & 4.9 & 300 & 0.9 & 0.97 & 8.0 & $<0.05$ & $<0.1$ \\
\hline
\end{tabular}


Table 4. Chemical analyses of soil samples from the western United States

\begin{tabular}{|c|c|c|c|c|c|c|c|c|c|}
\hline Field No. & $\mathrm{Sc}$ & $\mathrm{Se}$ & $\mathrm{Sm}$ & $\mathrm{Sn}$ & $\mathrm{Sr}$ & $\mathrm{Tb}$ & $\mathrm{Te}$ & $\mathrm{Th}$ & $\mathrm{Tl}$ \\
\hline & $\mathrm{ppm}$ & $\mathrm{ppm}$ & $\mathrm{ppm}$ & $\mathrm{ppm}$ & ppm & $\mathrm{ppm}$ & $\mathrm{ppm}$ & $\mathrm{ppm}$ & $\mathrm{ppm}$ \\
\hline LLD $^{1}$ & 0.3 & 1 & 0.02 & 0.5 & 0.05 & 0.005 & 0.1 & 0.03 & 0.1 \\
\hline CS01 & 11 & $<1$ & 8.3 & 4.0 & 210 & 1.1 & $<0.1$ & 16 & 0.7 \\
\hline $\mathrm{CS} 02$ & 9.6 & $<1$ & 6.4 & 3.0 & 210 & 0.84 & $<0.1$ & 12 & 0.7 \\
\hline CS03 & 12 & $<1$ & 9.2 & 3.0 & 140 & 0.99 & $<0.1$ & 16 & 0.8 \\
\hline CS04 & 12 & $<1$ & 7.6 & 3.0 & 260 & 0.96 & $<0.1$ & 14 & 0.7 \\
\hline CS05 & 4.0 & $<1$ & 3.6 & 1.0 & 400 & 0.48 & $<0.1$ & 6.6 & 0.4 \\
\hline CS06 & 6.1 & $<1$ & 7.1 & 19 & 100 & 0.87 & $<0.1$ & 29 & 3.9 \\
\hline CS07 & 7.7 & $<1$ & 7.5 & 2.0 & 150 & 0.89 & $<0.1$ & 18 & 1.9 \\
\hline CS08 & 12 & 1 & 5.9 & 2.0 & 290 & 0.78 & $<0.1$ & 8.8 & 1.1 \\
\hline CS09 & 10 & $<1$ & 6.8 & 2.0 & 190 & 0.86 & $<0.1$ & 9.8 & 0.9 \\
\hline GS10 & 9.2 & $<1$ & 5.4 & 2.0 & 140 & 0.69 & $<0.1$ & 9.8 & 0.7 \\
\hline CS11 & 8.7 & 2 & 4.5 & 2.0 & 740 & 0.6 & $<0.1$ & 8.6 & 1.3 \\
\hline CS12 & 11 & $<1$ & 5.0 & 2.0 & 170 & 0.61 & $<0.1$ & 8.8 & 0.5 \\
\hline CS13 & 12 & $<1$ & 4.8 & 2.0 & 150 & 0.60 & $<0.1$ & 8.0 & 0.5 \\
\hline CS14 & 5.9 & $<1$ & 3.8 & 1.0 & 610 & 0.44 & $<0.1$ & 5.9 & 0.3 \\
\hline CS15 & 4.0 & $<1$ & 2.4 & 0.90 & 780 & 0.33 & $<0.1$ & 3.6 & 0.4 \\
\hline CS16 & 15 & $<1$ & 6.6 & 4.0 & 100 & 0.70 & $<0.1$ & 19 & 1.0 \\
\hline CS17 & 12 & $<1$ & 19 & 3.0 & 78 & 1.8 & $<0.1$ & 43 & 1.1 \\
\hline CS18 & 14 & $<1$ & 10 & 3.0 & 530 & 1.1 & $<0.1$ & 22 & 0.6 \\
\hline CS19 & 16 & $<1$ & 9.1 & 3.0 & 590 & 1.0 & $<0.1$ & 18 & 0.5 \\
\hline S01 & 29 & $<1$ & 3.1 & 3.0 & 210 & 0.51 & $<0.1$ & 0.77 & 0.1 \\
\hline S02 & 34 & $<1$ & 3.2 & 2.0 & 230 & 0.56 & $<0.1$ & 0.72 & $<0.1$ \\
\hline S03 & 25 & $<1$ & 6.1 & 2.0 & 85 & 0.90 & $<0.1$ & 1.5 & $<0.1$ \\
\hline S04 & 33 & $<1$ & 2.8 & 3.0 & 96 & 0.53 & $<0.1$ & 0.47 & $<0.1$ \\
\hline S05 & 42 & $<1$ & 16 & 3.0 & 51 & 2.8 & $<0.1$ & 0.71 & 0.1 \\
\hline S06 & 31 & $<1$ & 4.2 & 3.0 & 80 & 0.76 & $<0.1$ & 0.73 & 0.3 \\
\hline S07 & 42 & $<1$ & 4.0 & 2.0 & 19 & 0.78 & $<0.1$ & 0.86 & 0.1 \\
\hline S08 & 28 & $<1$ & 3.1 & 1.0 & 99 & 0.58 & $<0.1$ & 0.54 & $<0.1$ \\
\hline S09 & 48 & $<1$ & 4.7 & 2.0 & 9.2 & 0.78 & $<0.1$ & 3.7 & $<0.1$ \\
\hline S10 & 30 & $<1$ & 6.2 & 2.0 & 340 & 0.93 & $<0.1$ & 1.4 & $<0.1$ \\
\hline S11 & 23 & $<1$ & 6.9 & 2.0 & 270 & 1.0 & $<0.1$ & 3.3 & $<0.1$ \\
\hline $\mathrm{S} 12$ & 37 & $<1$ & 2.1 & 2.0 & 100 & 0.40 & $<0.1$ & 0.53 & $<0.1$ \\
\hline S13 & 44 & $<1$ & 1.4 & 2.0 & 540 & 0.35 & $<0.1$ & 0.20 & $<0.1$ \\
\hline S14 & 26 & $<1$ & 2.7 & 4.0 & 800 & 0.56 & $<0.1$ & 0.30 & $<0.1$ \\
\hline S15 & 36 & $<1$ & 1.3 & 0.70 & 190 & 0.30 & $<0.1$ & 0.41 & $<0.1$ \\
\hline S16 & 44 & $<1$ & 1.4 & 1.0 & 480 & 0.33 & $<0.1$ & 0.44 & $<0.1$ \\
\hline S17 & 38 & $<1$ & 1.4 & 1.0 & 150 & 0.28 & $<0.1$ & 0.37 & $<0.1$ \\
\hline
\end{tabular}


Table 4. Chemical analyses of soil samples from the western United States

\begin{tabular}{|c|c|c|c|c|c|c|c|}
\hline Field No. & $\mathrm{Tm}$ & $\mathrm{U}$ & $\mathrm{V}$ & $\mathrm{W}$ & $\mathrm{Y}$ & $\mathrm{Yb}$ & $\mathrm{Zn}$ \\
\hline & ppm & $\mathrm{ppm}$ & ppm & $\mathrm{ppm}$ & ppm & ppm & ppm \\
\hline $\operatorname{LLD}^{1}$ & 0.006 & 0.02 & 0.4 & 0.1 & 0.3 & 0.03 & 5 \\
\hline CS01 & 0.72 & 2.7 & 79 & 2.6 & 45 & 4.3 & 80 \\
\hline $\mathrm{CS} 02$ & 0.55 & 2.6 & 70 & 4.7 & 34 & 3.1 & 84 \\
\hline CS03 & 0.42 & 2.9 & 81 & 2.4 & 31 & 2.4 & 120 \\
\hline CS04 & 0.63 & 2.7 & 98 & 2.3 & 41 & 3.6 & 93 \\
\hline CS05 & 0.33 & 4.7 & 37 & 0.9 & 20 & 1.8 & 36 \\
\hline CS06 & 0.53 & 14 & 49 & 4.8 & 37 & 3.0 & 130 \\
\hline CS07 & 0.5 & 6.0 & 57 & 2.5 & 34 & 2.9 & 88 \\
\hline CS08 & 0.54 & 6.4 & 120 & 1.3 & 34 & 3.2 & 160 \\
\hline CS09 & 0.53 & 2.9 & 110 & 1.3 & 36 & 3.0 & 100 \\
\hline GS10 & 0.44 & 3.6 & 95 & 1.2 & 29 & 2.5 & 93 \\
\hline CS11 & 0.4 & 5.9 & 170 & 1.3 & 27 & 2.4 & 120 \\
\hline CS12 & 0.38 & 2.7 & 96 & 1.5 & 23 & 2.3 & 87 \\
\hline CS13 & 0.36 & 2.3 & 100 & 1.3 & 22 & 2.1 & 100 \\
\hline CS14 & 0.27 & 2.9 & 41 & 0.6 & 17 & 1.5 & 32 \\
\hline CS15 & 0.21 & 5.3 & 32 & 0.3 & 14 & 1.3 & 10 \\
\hline CS16 & 0.35 & 4.1 & 89 & 1.8 & 25 & 2.0 & 50 \\
\hline CS17 & 0.6 & 8.2 & 79 & 1.7 & 48 & 3.4 & 75 \\
\hline CS18 & 0.6 & 4.1 & 120 & 1.9 & 41 & 3.3 & 120 \\
\hline CS19 & 0.61 & 4.1 & 140 & 2.3 & 41 & 3.5 & 150 \\
\hline S01 & 0.3 & 0.69 & 270 & 0.4 & 30 & 2.1 & 96 \\
\hline S02 & 0.34 & 0.64 & 290 & 0.2 & 36 & 2.4 & 100 \\
\hline S03 & 0.52 & 0.40 & 250 & 0.6 & 47 & 3.9 & 120 \\
\hline S04 & 0.31 & 0.64 & 180 & 0.4 & 32 & 2.1 & 93 \\
\hline S05 & 1.9 & 0.50 & 210 & 0.3 & 130 & 14 & 110 \\
\hline S06 & 0.49 & 0.40 & 200 & 0.5 & 42 & 3.6 & 130 \\
\hline S07 & 0.51 & 0.56 & 220 & 0.2 & 46 & 3.7 & 74 \\
\hline S08 & 0.36 & 0.40 & 210 & 0.2 & 36 & 2.5 & 66 \\
\hline S09 & 0.44 & 2.0 & 250 & $<0.1$ & 44 & 3.0 & 78 \\
\hline S10 & 0.53 & 0.80 & 300 & 0.2 & 48 & 3.7 & 93 \\
\hline S11 & 0.55 & 1.7 & 190 & 0.2 & 55 & 4.0 & 120 \\
\hline $\mathrm{S} 12$ & 0.26 & 0.30 & 150 & 0.2 & 24 & 1.8 & 93 \\
\hline S13 & 0.26 & 0.40 & 180 & $<0.1$ & 25 & 1.8 & 85 \\
\hline S14 & 0.38 & 0.20 & 110 & 0.1 & 44 & 2.7 & 68 \\
\hline S15 & 0.22 & 0.10 & 120 & $<0.1$ & 19 & 1.6 & 64 \\
\hline S16 & 0.25 & 0.10 & 160 & 0.1 & 23 & 1.8 & 72 \\
\hline S17 & 0.20 & 0.10 & 170 & $<0.1$ & 19 & 1.4 & 70 \\
\hline
\end{tabular}


Table 5. Chemical analyses of rocks from the western United States

\begin{tabular}{ccccccc}
\hline Field No. & $\begin{array}{c}\text { North Latitude } \\
\text { Degree }\end{array}$ & $\begin{array}{c}\text { East Longitude } \\
\text { Degree }\end{array}$ & Rock Type & $\begin{array}{c}\mathrm{Al} \\
\%\end{array}$ & $\begin{array}{c}\mathrm{Ca} \\
\%\end{array}$ & $\begin{array}{c}\mathrm{Fe} \\
\%\end{array}$ \\
\hline \hline LLD $^{1}$ & & & & 0.005 & 0.005 & 0.005 \\
CR02 & 38.5075 & 106.6638 & tuffaceous sedimentary rock & 6.6 & 0.99 & 0.42 \\
CR04 & 38.4050 & 107.0766 & ash-flow tuff & 6.6 & 0.61 & 1.1 \\
CR05 & 38.4159 & 107.0763 & ash-flow tuff & 2.6 & 17 & 0.85 \\
CR06 & 37.9266 & 107.4576 & mineralized ash-flow tuff & 6.1 & 0.2 & 0.95 \\
CR08 & 38.5590 & 107.3282 & andesite & 8.9 & 4.8 & 4.6 \\
CR11 & 38.5312 & 107.9370 & marine shale & 5.9 & 7.1 & 2.7 \\
CR12 & 39.0315 & 108.0592 & basalt & 7.4 & 5.8 & 7.9 \\
CR15 & 39.6643 & 106.9513 & evaporite & 0.34 & 20 & 0.26 \\
CR16 & 39.5188 & 106.3526 & clastic sedimentary rock & 2.1 & 0.76 & 2.0 \\
CR18 & 39.7511 & 106.1740 & andesite & 8.0 & 3.8 & 6.0 \\
\hline
\end{tabular}

${ }^{1}$ lower limit of determination 
Table 5. Chemical analyses of rocks from the western United States

\begin{tabular}{cccccccccc}
\hline Field No. & $\begin{array}{c}\mathrm{K} \\
\%\end{array}$ & $\begin{array}{c}\mathrm{Mg} \\
\%\end{array}$ & $\begin{array}{c}\mathrm{Na} \\
\%\end{array}$ & $\begin{array}{c}\mathrm{P} \\
\%\end{array}$ & $\begin{array}{c}\mathrm{Ti} \\
\%\end{array}$ & $\begin{array}{c}\mathrm{Ag} \\
\mathrm{ppm}\end{array}$ & $\begin{array}{c}\mathrm{As} \\
\mathrm{ppm}\end{array}$ & $\begin{array}{c}\mathrm{Au} \\
\mathrm{ppm}\end{array}$ & $\begin{array}{c}\mathrm{Ba} \\
\mathrm{ppm}\end{array}$ \\
\hline \hline LLD $^{1}$ & 0.005 & 0.005 & 0.005 & 0.01 & 0.005 & 3 & 0.5 & 0.05 & 0.5 \\
CR02 & 3.7 & 0.06 & 3.0 & 0.04 & 0.04 & $<3$ & 1.0 & $<0.05$ & 120 \\
CR04 & 4.3 & 0.13 & 2.3 & 0.04 & 0.20 & $<3$ & 2.0 & $<0.05$ & 640 \\
CR05 & 1.4 & 1.3 & 0.80 & 0.08 & 0.10 & $<3$ & 4.0 & $<0.05$ & 1100 \\
CR06 & 4.7 & 0.24 & 1.3 & 0.01 & 0.10 & $<3$ & 0.6 & $<0.05$ & 220 \\
CR08 & 2.1 & 0.84 & 2.6 & 0.24 & 0.80 & $<3$ & 1.0 & $<0.05$ & 970 \\
CR11 & 1.9 & 1.8 & 0.56 & 0.09 & 0.30 & $<3$ & 12 & $<0.05$ & 510 \\
CR12 & 1.2 & 4.6 & 2.1 & 0.16 & 1.2 & $<3$ & $<0.5$ & $<0.05$ & 940 \\
CR15 & 0.17 & 0.95 & 0.06 & $<0.01$ & 0.03 & $<3$ & $<0.5$ & 0.05 & 46 \\
CR16 & 0.65 & 0.55 & 0.02 & $<0.01$ & 0.02 & $<3$ & $<0.5$ & $<0.05$ & 2800 \\
CR18 & 3.6 & 1.9 & 2.2 & 0.23 & 0.60 & $<3$ & $<0.5$ & $<0.05$ & 1200 \\
\hline
\end{tabular}


Table 5. Chemical analyses of rocks from the western United States

\begin{tabular}{cccccccccc}
\hline Field No. & $\begin{array}{c}\mathrm{Be} \\
\mathrm{ppm}\end{array}$ & $\begin{array}{c}\mathrm{Bi} \\
\mathrm{ppm}\end{array}$ & $\begin{array}{c}\mathrm{Cd} \\
\mathrm{ppm}\end{array}$ & $\begin{array}{c}\mathrm{Ce} \\
\mathrm{ppm}\end{array}$ & $\begin{array}{c}\mathrm{Co} \\
\mathrm{ppm}\end{array}$ & $\begin{array}{c}\mathrm{Cr} \\
\mathrm{ppm}\end{array}$ & $\begin{array}{c}\mathrm{Cs} \\
\mathrm{ppm}\end{array}$ & $\begin{array}{c}\mathrm{Cu} \\
\mathrm{ppm}\end{array}$ & $\begin{array}{c}\mathrm{Dy} \\
\mathrm{ppm}\end{array}$ \\
\hline \hline LLD $^{1}$ & $<0.1$ & 0.05 & 0.1 & 0.5 & 0.1 & 0.2 & 0.01 & 3 & 0.04 \\
CR02 & 8.3 & 0.30 & $<0.1$ & 18 & 0.6 & 2 & 4.4 & $<3$ & 6.4 \\
CR04 & 1.8 & $<0.05$ & $<0.1$ & 89 & 2.3 & 2 & 2.6 & 5 & 6.0 \\
CR05 & 1.0 & $<0.05$ & 0.2 & 42 & 2.4 & 6 & 1.6 & 6 & 3.5 \\
CR06 & 4.4 & 0.20 & 0.1 & 98 & 1.7 & 2 & 7.8 & 7 & 4.9 \\
CR08 & 1.6 & $<0.05$ & 0.1 & 82 & 12 & 3 & 1.2 & 9 & 7.9 \\
CR11 & 1.6 & 0.20 & 1.0 & 50 & 7.9 & 73 & 9.1 & 20 & 4.2 \\
CR12 & 1.2 & $<0.05$ & $<0.1$ & 62 & 47 & 300 & 0.40 & 52 & 6.0 \\
CR15 & $<0.1$ & $<0.05$ & 0.3 & 5 & 0.6 & 5 & 0.30 & $<3$ & 0.39 \\
CR16 & 0.2 & $<0.05$ & $<0.1$ & 29 & 5.4 & 10 & 0.40 & $<3$ & 0.83 \\
CR18 & 3.5 & $<0.05$ & 0.3 & 88 & 22 & 37 & 2.5 & 71 & 6.3 \\
\hline
\end{tabular}


Table 5. Chemical analyses of rocks from the western United States

\begin{tabular}{cccccccccc}
\hline Field No. & $\begin{array}{c}\text { Er } \\
\text { ppm }\end{array}$ & $\begin{array}{c}\text { Eu } \\
\text { ppm }\end{array}$ & $\begin{array}{c}\text { Ga } \\
\text { ppm }\end{array}$ & $\begin{array}{c}\text { Gd } \\
\text { ppm }\end{array}$ & $\begin{array}{c}\text { Ge } \\
\text { ppm }\end{array}$ & $\begin{array}{c}\text { Ho } \\
\text { ppm }\end{array}$ & $\begin{array}{c}\text { In } \\
\text { ppm }\end{array}$ & $\begin{array}{c}\text { La } \\
\text { ppm }\end{array}$ & $\begin{array}{c}\text { Li } \\
\text { ppm }\end{array}$ \\
\hline \hline LLD $^{1}$ & 0.05 & 0.005 & 0.006 & 0.02 & 0.1 & 0.02 & 0.1 & 0.3 & 0.2 \\
CR02 & 3.2 & 0.24 & 27 & 4.3 & 2.4 & 1.2 & 0.1 & 10 & 88 \\
CR04 & 3.6 & 1.4 & 15 & 5.9 & 1.1 & 1.3 & $<0.1$ & 55 & 26 \\
CR05 & 2.0 & 0.84 & 6.4 & 3.7 & 0.4 & 0.73 & $<0.1$ & 31 & 17 \\
CR06 & 3.1 & 0.47 & 18 & 4.2 & 1.0 & 1.0 & $<0.1$ & 53 & 22 \\
CR08 & 4.4 & 2.9 & 23 & 8.3 & 1.0 & 1.6 & $<0.1$ & 42 & 12 \\
CR11 & 2.4 & 1.1 & 16 & 4.1 & 0.9 & 0.88 & $<0.1$ & 30 & 37 \\
CR12 & 3.4 & 2.0 & 20 & 5.9 & 1.0 & 1.2 & $<0.1$ & 34 & 8.1 \\
CR15 & 0.23 & 0.10 & 0.90 & 0.37 & 0.1 & 0.08 & $<0.1$ & 2.6 & 4.0 \\
CR16 & 0.36 & 0.38 & 4.8 & 1.6 & 0.9 & 0.14 & $<0.1$ & 13 & 22 \\
CR18 & 3.7 & 2.1 & 21 & 6.3 & 1.1 & 1.3 & $<0.1$ & 49 & 17 \\
\hline
\end{tabular}


Table 5. Chemical analyses of rocks from the western United States

\begin{tabular}{cccccccccc}
\hline Field No. & $\begin{array}{c}\mathrm{Mn} \\
\mathrm{ppm}\end{array}$ & $\begin{array}{c}\mathrm{Mo} \\
\mathrm{ppm}\end{array}$ & $\begin{array}{c}\mathrm{Nb} \\
\mathrm{ppm}\end{array}$ & $\begin{array}{c}\mathrm{Nd} \\
\mathrm{ppm}\end{array}$ & $\begin{array}{c}\mathrm{Ni} \\
\mathrm{ppm}\end{array}$ & $\begin{array}{c}\mathrm{Pb} \\
\mathrm{ppm}\end{array}$ & $\begin{array}{c}\mathrm{Pr} \\
\mathrm{ppm}\end{array}$ & $\begin{array}{c}\mathrm{Rb} \\
\mathrm{ppm}\end{array}$ & $\begin{array}{c}\mathrm{Re} \\
\mathrm{ppm}\end{array}$ \\
\hline \hline LLD $^{1}$ & 0.2 & 0.1 & 1 & 0.2 & 1 & 0.8 & 0.05 & 0.01 & 0.05 \\
CR02 & 1300 & 1.3 & 60 & 11 & 1.4 & 34 & 2.8 & 260 & $<0.05$ \\
CR04 & 460 & 0.6 & 23 & 41 & 3.6 & 21 & 11 & 150 & $<0.05$ \\
CR05 & 220 & 0.5 & 8.2 & 24 & 7.1 & 8.2 & 6.1 & 52 & $<0.05$ \\
CR06 & 510 & 1.2 & 61 & 31 & 1.6 & 20 & 9.5 & 360 & $<0.05$ \\
CR08 & 1000 & 0.7 & 16 & 46 & 1.7 & 12 & 11 & 64 & $<0.05$ \\
CR11 & 170 & 19 & 15 & 25 & 42 & 16 & 6.4 & 110 & 0.10 \\
CR12 & 1200 & 1.2 & 22 & 33 & 160 & 7.5 & 7.9 & 25 & $<0.05$ \\
CR15 & 55 & 3.7 & 1.3 & 2.4 & 2.3 & 2.8 & 0.60 & 6.0 & $<0.05$ \\
CR16 & 240 & 0.1 & 1.1 & 13 & 6.3 & 0.6 & 3.6 & 24 & $<0.05$ \\
CR18 & 1200 & 1.5 & 34 & 41 & 13 & 22 & 10 & 160 & $<0.05$ \\
\hline
\end{tabular}


Table 5. Chemical analyses of rocks from the western United States

\begin{tabular}{cccccccccc}
\hline Field No. & $\begin{array}{c}\mathrm{Sb} \\
\mathrm{ppm}\end{array}$ & $\begin{array}{c}\mathrm{Sc} \\
\mathrm{ppm}\end{array}$ & $\begin{array}{c}\mathrm{Se} \\
\mathrm{ppm}\end{array}$ & $\begin{array}{c}\mathrm{Sm} \\
\mathrm{ppm}\end{array}$ & $\begin{array}{c}\mathrm{Sn} \\
\mathrm{ppm}\end{array}$ & $\begin{array}{c}\mathrm{Sr} \\
\mathrm{ppm}\end{array}$ & $\begin{array}{c}\mathrm{Tb} \\
\mathrm{ppm}\end{array}$ & $\begin{array}{c}\mathrm{Te} \\
\mathrm{ppm}\end{array}$ & $\begin{array}{c}\mathrm{Th} \\
\mathrm{ppm}\end{array}$ \\
\hline \hline LLD $^{1}$ & 0.1 & 0.3 & 1 & 0.02 & 0.5 & 0.05 & 0.005 & 0.1 & 0.03 \\
CR02 & 0.2 & 8.6 & $<1$ & 3.8 & 6.0 & 27 & 0.91 & $<0.1$ & 12 \\
CR04 & 0.3 & 4.0 & $<1$ & 7.5 & 1.0 & 120 & 0.91 & $<0.1$ & 18 \\
CR05 & 0.6 & 2.0 & $<1$ & 4.4 & 0.7 & 420 & 0.55 & $<0.1$ & 6.7 \\
CR06 & 0.5 & 2.0 & $<1$ & 5.5 & 3.0 & 61 & 0.70 & $<0.1$ & 45 \\
CR08 & 0.1 & 12 & $<1$ & 9.6 & 2.0 & 1000 & 1.2 & $<0.1$ & 4.7 \\
CR11 & 1.3 & 9.6 & 6 & 5.0 & 2.0 & 300 & 0.64 & $<0.1$ & 9.2 \\
CR12 & $<0.1$ & 25 & $<1$ & 6.6 & 2.0 & 720 & 0.91 & $<0.1$ & 3.7 \\
CR15 & $<0.1$ & 0.6 & $<1$ & 0.40 & $<0.5$ & 1100 & 0.06 & $<0.1$ & 0.71 \\
CR16 & $<0.1$ & 1.0 & $<1$ & 2.7 & 0.7 & 80 & 0.16 & $<0.1$ & 4.2 \\
CR18 & 0.2 & 20 & $<1$ & 7.9 & 2.0 & 1200 & 0.94 & $<0.1$ & 15 \\
\hline
\end{tabular}


Table 5. Chemical analyses of rocks from the western United States

\begin{tabular}{ccccccccc}
\hline Field No. & $\begin{array}{c}\mathrm{Tl} \\
\mathrm{ppm}\end{array}$ & $\begin{array}{c}\mathrm{Tm} \\
\mathrm{ppm}\end{array}$ & $\begin{array}{c}\mathrm{U} \\
\mathrm{ppm}\end{array}$ & $\begin{array}{c}\mathrm{V} \\
\mathrm{ppm}\end{array}$ & $\begin{array}{c}\mathrm{W} \\
\mathrm{ppm}\end{array}$ & $\begin{array}{c}\mathrm{Y} \\
\mathrm{ppm}\end{array}$ & $\begin{array}{c}\mathrm{Yb} \\
\mathrm{ppm}\end{array}$ & $\begin{array}{c}\mathrm{Zn} \\
\mathrm{ppm}\end{array}$ \\
\hline \hline LLD $^{1}$ & 0.1 & 0.006 & 0.02 & 0.4 & 0.1 & 0.3 & 0.03 & 5 \\
$\mathrm{CR} 02$ & 1.5 & 0.53 & 4.2 & 5.0 & 1.7 & 42 & 3.0 & 77 \\
$\mathrm{CR} 04$ & 0.6 & 0.61 & 4.0 & 15 & 1.2 & 37 & 3.7 & 40 \\
$\mathrm{CR} 05$ & 0.2 & 0.33 & 5.4 & 21 & 0.8 & 23 & 1.9 & 20 \\
$\mathrm{CR} 06$ & 3.6 & 0.56 & 14 & 12 & 3.7 & 33 & 3.4 & 57 \\
$\mathrm{CR} 08$ & 0.4 & 0.73 & 1.7 & 100 & 0.6 & 48 & 4.2 & 140 \\
$\mathrm{CR} 11$ & 1.8 & 0.41 & 6.6 & 210 & 1.6 & 28 & 2.4 & 120 \\
$\mathrm{CR} 12$ & 0.1 & 0.54 & 0.88 & 190 & 0.4 & 37 & 3.0 & 110 \\
CR15 & $<0.1$ & 0.04 & 1.2 & 6.0 & $<0.1$ & 2.4 & 0.20 & 20 \\
CR16 & $<0.1$ & 0.07 & 0.69 & 12 & 0.2 & 3.8 & 0.30 & 7 \\
CR18 & 0.2 & 0.62 & 4.0 & 160 & 1.5 & 39 & 3.6 & 110 \\
\hline
\end{tabular}

${ }^{1}$ lower limit of determination 
Table 6. Chemical analyses of water-soluble extractions of soils from Guam

\begin{tabular}{|c|c|c|c|c|c|c|c|c|}
\hline Site No. & Formation & $\mathrm{pH}$ & $\begin{array}{c}\mathrm{A} 1 \\
\mathrm{ppm} \\
\end{array}$ & $\begin{array}{c}\mathrm{Ca} \\
\mathrm{ppm}\end{array}$ & $\begin{array}{c}\mathrm{Na} \\
\mathrm{ppm}\end{array}$ & $\begin{array}{r}\mathrm{Mg} \\
\mathrm{ppm}\end{array}$ & $\begin{array}{c}\mathrm{K} \\
\mathrm{ppm}\end{array}$ & $\begin{array}{l}\mathrm{Mn} \\
\mathrm{ppm}\end{array}$ \\
\hline $\mathrm{LLD}^{1}$ & & & 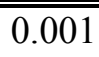 & 0.5 & 0.1 & 0.1 & 0.3 & 0.0001 \\
\hline GS-01 & Facpi, tuff & 5.97 & 5.7 & 83 & 66 & 31 & 46 & 0.71 \\
\hline GS-02 & Facpi, tuff & 6.69 & 3.0 & 137 & 61 & 15 & 33 & 0.22 \\
\hline GS-03 & Facpi, tuff & 6.01 & 4.1 & 66 & 69 & 17 & 64 & 1.34 \\
\hline GS-04 & Facpi, tuff & 5.90 & 6.0 & 83 & 46 & 17 & 24 & 0.95 \\
\hline GS-05 & Facpi, tuff & 6.28 & 2.5 & 156 & 55 & 23 & 40 & 0.59 \\
\hline GS-06 & Facpi, tuff & 6.19 & 4.1 & 228 & 54 & 42 & 54 & 2.43 \\
\hline GS-07 & Facpi, tuff & 6.00 & 2.9 & 71 & 53 & 7.6 & 14 & 0.45 \\
\hline GS-08 & Facpi, tuff & 7.60 & 3.1 & 170 & 51 & 8.7 & 30 & 0.24 \\
\hline GS-09 & Bolanos & 5.08 & 0.79 & 1.1 & 98 & 6.1 & 8.3 & 0.97 \\
\hline GS-10 & Bolanos & 6.32 & 1.7 & 17 & 77 & 22 & 13 & 0.13 \\
\hline GS-11 & Bolanos & 6.72 & 9.9 & 9.4 & 125 & 11 & 31 & 0.58 \\
\hline GS-12 & Facpi, basalt & 7.53 & 3.6 & 139 & 37 & 36 & 33 & 0.26 \\
\hline GS-13 & Facpi, basalt & 7.44 & 5.7 & 154 & 39 & 19 & 14 & 0.36 \\
\hline GS-14 & Facpi, basalt & 7.71 & 12.2 & 376 & 54 & 23 & 74 & 0.94 \\
\hline GS-15 & Facpi, basalt & 7.11 & 6.1 & 24 & 107 & 23 & 9.0 & 0.29 \\
\hline GS-16 & Facpi, basalt & 7.32 & 2.8 & 15 & 38 & 13 & 3.7 & 0.15 \\
\hline GS-17 & Facpi, basalt & 7.13 & 3.7 & 12 & 46 & 11 & 4.3 & 0.12 \\
\hline GS-18 & Limestone, undivided & 7.58 & 4.0 & 106 & 16 & 1.7 & 11 & 0.68 \\
\hline GS-19 & Alutom & 7.96 & 3.1 & 171 & 23 & 10 & 21 & 0.16 \\
\hline GS-20 & Alutom & 5.81 & 3.1 & 17 & 40 & 7.8 & 24 & 0.49 \\
\hline GS-21 & Alutom & 6.17 & 1.2 & 9.4 & 23 & 13 & 10 & 0.13 \\
\hline GS-22 & Alutom & 5.59 & 3.7 & 8.4 & 40 & 10 & 12 & 0.81 \\
\hline GS-23 & Facpi, basalt & 6.76 & 5.5 & 11 & 87 & 7.7 & 6.6 & 0.26 \\
\hline GS-24 & Facpi, basalt & 6.19 & 10.0 & 87 & 85 & 50 & 33 & 0.80 \\
\hline GS-25 & Facpi, basalt & 7.25 & 8.9 & 17 & 96 & 24 & 4.1 & 0.33 \\
\hline GS-09R ${ }^{2}$ & Bolanos & & 0.87 & 1.1 & 102 & 6.1 & 8.2 & 1.10 \\
\hline GS-17R ${ }^{2}$ & Facpi, basalt & & 1.8 & 11 & 43 & 8.4 & 3.9 & 0.07 \\
\hline GS-21R ${ }^{2}$ & Alutom & & 1.1 & 9.4 & 21 & 11 & 10 & 0.13 \\
\hline
\end{tabular}

${ }^{1}$ lower limit of determinatin

${ }^{2}$ duplicate 
Table 6. Chemical analyses of water-soluble extractions of soils from Guam

\begin{tabular}{|c|c|c|c|c|c|c|c|c|c|c|c|}
\hline Site No. & $\begin{array}{c}\mathrm{Fe} \\
\mathrm{ppm}\end{array}$ & $\begin{array}{c}\mathrm{Si} \\
\mathrm{ppm}\end{array}$ & $\begin{array}{c}\mathrm{P} \\
\mathrm{ppm}\end{array}$ & $\begin{array}{l}\mathrm{SO}_{4} \\
\mathrm{ppm}\end{array}$ & $\begin{array}{r}\mathrm{Li} \\
\mathrm{ppb} \\
\end{array}$ & $\begin{array}{c}\mathrm{Be} \\
\mathrm{ppb}\end{array}$ & $\begin{array}{c}\mathrm{V} \\
\mathrm{ppb}\end{array}$ & $\begin{array}{c}\mathrm{Cr} \\
\mathrm{ppb} \\
\end{array}$ & $\begin{array}{r}\mathrm{Co} \\
\mathrm{ppb}\end{array}$ & $\begin{array}{c}\mathrm{Ni} \\
\mathrm{ppb}\end{array}$ & $\begin{array}{r}\mathrm{Cu} \\
\mathrm{ppb} \\
\end{array}$ \\
\hline $\mathrm{LLD}^{1}$ & 0.5 & 0.2 & $\overline{0} 0.1$ & 20 & 1 & 0.5 & 1 & 10 & 0.2 & 1 & 5 \\
\hline GS-01 & 6.5 & 53 & 8.2 & 51 & 24 & 0.5 & 100 & 25 & 11 & 128 & 465 \\
\hline GS-02 & 2.0 & 36 & 5.5 & 78 & $<1$ & $<0.5$ & 32 & 11 & 6 & 47 & 281 \\
\hline GS-03 & 5.8 & 37 & 6.0 & 40 & 10 & 1.5 & 49 & 11 & 9 & 43 & 285 \\
\hline GS-04 & 6.7 & 38 & 5.5 & 34 & 19 & 0.5 & 31 & 28 & 30 & 160 & 402 \\
\hline GS-05 & 3.5 & 45 & 11 & 60 & 10 & 0.6 & 30 & 20 & 10 & 55 & 181 \\
\hline GS-06 & 12.3 & 53 & 14 & 98 & 8 & $<0.5$ & 55 & 16 & 22 & 51 & 292 \\
\hline GS-07 & 2.8 & 44 & 6.2 & 38 & 6 & $<0.5$ & 18 & 12 & 8 & 67 & 232 \\
\hline GS-08 & 1.1 & 25 & 5.0 & 26 & $<1$ & $<0.5$ & 41 & $<10$ & 5 & 17 & 61 \\
\hline GS-09 & 0.5 & 21 & 7.0 & $<20$ & 6 & $<0.5$ & 3 & $<10$ & 44 & 5 & 135 \\
\hline GS-10 & 1.1 & 33 & 4.7 & $<20$ & $<1$ & $<0.5$ & 18 & $<10$ & 3 & 12 & 68 \\
\hline GS-11 & 8.5 & 56 & 9.7 & 27 & 3 & 1.6 & 90 & 12 & 14 & 168 & 297 \\
\hline GS-12 & 2.1 & 28 & 6.4 & 38 & 14 & $<0.5$ & 27 & 26 & 8 & 98 & 197 \\
\hline GS-13 & 3.8 & 42 & 5.3 & 35 & $<1$ & $<0.5$ & 72 & 21 & 13 & 81 & 108 \\
\hline GS-14 & 8.1 & 47 & 12 & 88 & 6 & $<0.5$ & 71 & 46 & 23 & 198 & 466 \\
\hline GS-15 & 5.2 & 38 & 3.9 & 28 & $<1$ & $<0.5$ & 53 & 27 & 9 & 67 & 181 \\
\hline GS-16 & 1.9 & 37 & 3.0 & 20 & $<1$ & $<0.5$ & 76 & 21 & 4 & 44 & 176 \\
\hline GS-17 & 2.7 & 30 & 5.6 & $<20$ & $<1$ & $<0.5$ & 71 & 14 & 5 & 47 & 81 \\
\hline GS-18 & 0.4 & $<0.2$ & 3.4 & 21 & $<1$ & 0.6 & 11 & 146 & 8 & 48 & 20 \\
\hline GS-19 & 3.3 & 29 & 4.7 & 30 & 4 & $<0.5$ & 58 & 13 & 5 & 44 & 136 \\
\hline GS-20 & 0.5 & 12 & 5.2 & 34 & 2 & $<0.5$ & 10 & $<10$ & 14 & 9 & 115 \\
\hline GS-21 & 0.7 & 23 & 3.0 & $<20$ & $<1$ & $<0.5$ & 9 & $<10$ & 2 & 8 & 32 \\
\hline GS-22 & 0.5 & 14 & 7.9 & 27 & 1 & $<0.5$ & 4 & $<10$ & 22 & 11 & 64 \\
\hline GS-23 & 5.3 & 40 & 4.4 & 22 & $<1$ & $<0.5$ & 139 & 49 & 10 & 122 & 193 \\
\hline GS-24 & 11.3 & 51 & 5.0 & 41 & $<1$ & 0.5 & 82 & 52 & 23 & 211 & 174 \\
\hline GS-25 & 8.9 & 42 & 4.9 & 40 & 11 & $<0.5$ & 95 & 23 & 15 & 104 & 97 \\
\hline GS-09R ${ }^{2}$ & 0.6 & 23 & 5.7 & 33 & 6 & $<0.5$ & 3 & $<10$ & 48 & 5 & 128 \\
\hline GS-17R ${ }^{2}$ & 1.1 & 26 & 5.2 & $<20$ & $<1$ & $<0.5$ & 67 & 11 & 3 & 29 & 207 \\
\hline GS-21R & 0.7 & 23 & 2.9 & $<20$ & $<1$ & $<0.5$ & 9 & $<10$ & 3 & 14 & 31 \\
\hline
\end{tabular}

${ }^{1}$ lower limit of determinatin

${ }^{2}$ duplicate 
Table 6. Chemical analyses of water-soluble extractions of soils from Guam

\begin{tabular}{|c|c|c|c|c|c|c|c|c|c|c|}
\hline Site No. & $\begin{array}{c}\mathrm{Zn} \\
\mathrm{ppb} \\
\end{array}$ & $\begin{array}{c}\mathrm{As} \\
\mathrm{ppb}\end{array}$ & $\begin{array}{c}\mathrm{Se} \\
\mathrm{ppb}\end{array}$ & $\begin{array}{l}\mathrm{Mo} \\
\mathrm{ppb}\end{array}$ & $\begin{array}{c}\mathrm{Cd} \\
\mathrm{ppb} \\
\end{array}$ & $\begin{array}{c}\mathrm{Sb} \\
\mathrm{ppb}\end{array}$ & $\begin{array}{r}\mathrm{Ba} \\
\mathrm{ppb}\end{array}$ & $\begin{array}{r}\mathrm{Pb} \\
\mathrm{ppb} \\
\end{array}$ & $\begin{array}{l}\text { Th } \\
\mathrm{ppb}\end{array}$ & $\begin{array}{c}\mathrm{U} \\
\mathrm{ppb}\end{array}$ \\
\hline$\overline{L L L D}^{1}$ & $\overline{5}$ & 10 & 10 & 2 & 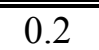 & 1 & 1 & $\overline{0.5}$ & 0.05 & 0.05 \\
\hline GS-01 & 382 & 60 & 11 & 8 & 7.2 & 11 & 72 & 75 & 5.4 & 1.2 \\
\hline GS-02 & 110 & $<10$ & 16 & 6 & 1.2 & 5 & 76 & 22 & 1.4 & 0.40 \\
\hline GS-03 & 139 & 11 & $<10$ & 3 & 2.6 & 2 & 350 & 25 & 2.7 & 0.20 \\
\hline GS-04 & 167 & $<10$ & 11 & 3 & 2.6 & 3 & 487 & 20 & 2.4 & 0.32 \\
\hline GS-05 & 94 & $<10$ & 20 & 3 & 2.9 & 2 & 100 & 13 & 3.6 & 0.05 \\
\hline GS-06 & 149 & $<10$ & 20 & 3 & 2.4 & 2 & 234 & 21 & 3.9 & 0.28 \\
\hline GS-07 & 893 & $<10$ & 16 & 6 & 11 & 3 & 46 & 17 & 0.98 & $<0.05$ \\
\hline GS-08 & 35 & $<10$ & $<10$ & 3 & 0.7 & 2 & 70 & 4.0 & 1.5 & 0.42 \\
\hline GS-09 & 19 & $<10$ & $<10$ & $<2$ & $<0.2$ & $<1$ & 87 & 1.5 & 0.28 & $<0.05$ \\
\hline GS-10 & 26 & $<10$ & $<10$ & 2 & 0.2 & 2 & 23 & 2.3 & 0.86 & 0.10 \\
\hline GS-11 & 1620 & $<10$ & $<10$ & 22 & 7.1 & 2 & 99 & 6.8 & 2.2 & 2.0 \\
\hline GS-12 & 31 & $<10$ & $<10$ & 3 & 0.5 & 1 & 117 & 4.5 & 1.0 & 0.31 \\
\hline GS-13 & 96 & 14 & $<10$ & 4 & 0.9 & 1 & 8 & 20 & 1.3 & 0.49 \\
\hline GS-14 & 107 & 13 & 21 & 7 & 1.5 & 3 & 98 & 19 & 2.6 & 0.58 \\
\hline GS-15 & 49 & $<10$ & $<10$ & $<2$ & 0.4 & 2 & 9 & 20 & 0.58 & 0.13 \\
\hline GS-16 & 237 & $<10$ & $<10$ & 4 & 1.5 & 2 & 6 & 11 & 0.46 & 0.27 \\
\hline GS-17 & 34 & $<10$ & $<10$ & 3 & 0.4 & 1 & 4 & 22 & 0.76 & 0.06 \\
\hline GS-18 & 16 & $<10$ & 10 & $<2$ & 0.7 & $<1$ & 3 & 6.9 & 0.27 & 0.99 \\
\hline GS-19 & 18 & $<10$ & $<10$ & 5 & 0.3 & 1 & 67 & 2.3 & 0.84 & 0.90 \\
\hline GS-20 & 12 & $<10$ & $<10$ & $<2$ & $<.2$ & $<1$ & 103 & 2.5 & 0.38 & 0.15 \\
\hline GS-21 & 11 & $<10$ & $<10$ & $<2$ & $<.2$ & $<1$ & 132 & 2.1 & 0.18 & 0.48 \\
\hline GS-22 & 6 & $<10$ & 13 & $<2$ & 0.2 & 2 & 60 & 1.8 & 0.30 & $<0.05$ \\
\hline GS-23 & 66 & $<10$ & $<10$ & 3 & 0.8 & 2 & 14 & 16 & 0.68 & 0.36 \\
\hline GS-24 & 108 & $<10$ & $<10$ & 3 & 2.7 & 2 & 33 & 9.0 & 0.92 & 0.63 \\
\hline GS-25 & 25 & $<10$ & $<10$ & $<2$ & 0.2 & $<1$ & 9 & 2.1 & 0.56 & $<0.05$ \\
\hline GS-09R ${ }^{2}$ & 14 & $<10$ & $<10$ & $<2$ & $<2$ & $<1$ & 87 & 0.7 & 0.10 & $<0.05$ \\
\hline GS-17R 2 & 74 & $<10$ & $<10$ & 3 & 0.9 & 1 & 2 & 3.4 & 0.45 & $<0.05$ \\
\hline GS-21R ${ }^{2}$ & 14 & $<10$ & $<10$ & $<2$ & 0.3 & $<1$ & 131 & 2.8 & 0.12 & $<0.05$ \\
\hline
\end{tabular}

${ }^{1}$ lower limit of determinatin

${ }^{2}$ duplicate 
Table 7. Chemical analyses of water-soluble extractions of soils from the western United States

\begin{tabular}{|c|c|c|c|c|c|c|c|c|}
\hline Site No. & Rock Type & $\mathrm{pH}$ & $\begin{array}{c}\mathrm{Al} \\
\mathrm{ppm}\end{array}$ & $\begin{array}{c}\mathrm{Ca} \\
\mathrm{ppm}\end{array}$ & $\begin{array}{c}\mathrm{Na} \\
\mathrm{ppm}\end{array}$ & $\begin{array}{c}\mathrm{Mg} \\
\mathrm{ppm}\end{array}$ & $\begin{array}{c}\mathrm{K} \\
\mathrm{ppm}\end{array}$ & $\begin{array}{c}\mathrm{Mn} \\
\mathrm{ppm}\end{array}$ \\
\hline $\mathrm{LLD}^{\top}$ & & & 0.001 & 0.5 & 0.1 & 0.1 & 0.3 & 0.0001 \\
\hline CS-1 & Tuffaceous sediments & 8.22 & 2.17 & 75 & 1.4 & 7.7 & 29 & 0.100 \\
\hline $\mathrm{CS}-2$ & Tuffaceous sediments & 8.62 & 1.68 & 86 & 1.4 & 5.8 & 17 & 0.110 \\
\hline CS-3 & Tuffaceous sediments & 7.15 & 1.66 & 39 & 2.2 & 7.2 & 38 & 0.269 \\
\hline CS-4 & Ash flow tuff & 8.27 & 1.34 & 28 & 15 & 4.4 & 12 & 0.052 \\
\hline CS-5 & Ash flow tuff & 8.73 & 0.79 & 121 & 27 & 26 & 3 & 0.022 \\
\hline CS-6 & Mineralized tuff breccia & 7.17 & 1.17 & 111 & 3.1 & 11 & 14 & 0.154 \\
\hline CS-7 & Mineralized tuff breccia & 7.72 & 1.59 & 60 & 3.5 & 7.6 & 19 & 0.238 \\
\hline CS-8 & Andesite & 7.13 & 3.35 & 73 & 32 & 11 & 97 & 0.263 \\
\hline CS-9 & Andesite & 6.99 & 2.66 & 58 & 11 & 7.6 & 101 & 0.445 \\
\hline CS-10 & Marine shale & 8.42 & 1.42 & 115 & 12 & 15 & 70 & 0.035 \\
\hline CS-11 & Marine shale & 7.92 & 0.35 & 6140 & 168 & 221 & 148 & 0.182 \\
\hline CS-12 & Basalt & 8.28 & 2.01 & 35 & 3.1 & 9.6 & 10 & 0.109 \\
\hline CS-13 & Basalt & 7.81 & 3.74 & 17 & 3.1 & 3.8 & 11 & 0.168 \\
\hline CS-14 & Evaporite & 8.61 & 0.66 & 7700 & $<0.1$ & 1960 & 410 & 0.110 \\
\hline CS-15 & Evaporite & 8.71 & 0.74 & 5460 & 200 & 784 & 93 & 0.081 \\
\hline CS-16 & Clastic sedimentary rock & 8.80 & 1.18 & 118 & 9.1 & 49 & 53 & 0.109 \\
\hline CS-17 & Clastic sedimentary rock & 8.77 & 1.22 & 35 & 2.1 & 3.7 & 14 & 0.320 \\
\hline CS-18 & Basalt & 7.83 & 1.64 & 28 & 6.1 & 9.2 & 22 & 0.263 \\
\hline CS-19 & Basalt & 7.48 & 2.73 & 26 & 3.7 & 7.3 & 29 & 0.413 \\
\hline S01 & Andesite & 8.78 & 3.47 & 45 & 4.4 & 4.4 & 118 & 0.458 \\
\hline S02 & Ash flow tuff & 8.68 & 1.98 & 81 & 5.2 & 7.4 & 50 & 0.229 \\
\hline S03 & Andesite & 8.21 & 2.91 & 20 & 3.1 & 4.3 & 13 & 0.170 \\
\hline S04 & Ash flow tuff & 8.11 & 4.30 & 89 & 8.4 & 11 & 46 & 0.510 \\
\hline S05 & Ash flow tuff & 8.52 & 4.82 & 77 & 2.3 & 4.0 & 40 & 0.228 \\
\hline S06 & Andesite & 8.20 & 3.92 & 38 & 3.9 & 4.4 & 27 & 0.439 \\
\hline S07 & Dacite and rhyodacite & 8.41 & 3.56 & 42 & 2.6 & 4.5 & 49 & 0.371 \\
\hline S08 & Basalt & 8.61 & 2.98 & 118 & 2.7 & 7.9 & 26 & 0.090 \\
\hline S09 & Basalt & 8.52 & 4.67 & 154 & 24 & 9.3 & 46 & 0.239 \\
\hline $\mathrm{S} 10$ & Andesite & 8.78 & 3.83 & 118 & 14 & 7.1 & 62 & 0.141 \\
\hline S11 & Andesite & 8.72 & 2.71 & 72 & 4.6 & 7.4 & 54 & 0.200 \\
\hline S12 & Andesite & 8.65 & 2.81 & 60 & 2.5 & 15 & 237 & 0.382 \\
\hline $\mathrm{S} 13$ & Dacite and rhyodacite & 8.44 & 1.41 & 144 & 3.3 & 7.6 & 22 & 0.074 \\
\hline S14 & Dacite and rhyodacite & 9.09 & 2.17 & 79 & 2.8 & 4.1 & 21 & 0.053 \\
\hline S15 & Basalt & 8.06 & 1.25 & 34 & 3.2 & 5.7 & 34 & 0.115 \\
\hline S16 & Basalt & 7.58 & 1.14 & 43 & 3.7 & 5.5 & 28 & 0.130 \\
\hline S17 & Andesite & 7.96 & 1.75 & 65 & 5.6 & 4.9 & 11 & 0.097 \\
\hline S01-R ${ }^{2}$ & Andesite & & 1.73 & 40 & 4.0 & 3.4 & 114 & 0.240 \\
\hline $\mathrm{CS}-1 \mathrm{R}^{2}$ & Tuffaceous sediments & & 1.50 & 75 & 1.2 & 6.8 & 28 & 0.070 \\
\hline
\end{tabular}

${ }^{1}$ lower limit of determinatin

${ }^{2}$ duplicate 
Table 7. Chemical analyses of water-soluble extractions of soils from the western United States

\begin{tabular}{|c|c|c|c|c|c|c|c|c|c|c|}
\hline Site No. & $\begin{array}{c}\mathrm{Fe} \\
\mathrm{ppm}\end{array}$ & $\begin{array}{c}\mathrm{Si} \\
\mathrm{ppm}\end{array}$ & $\begin{array}{c}\mathrm{P} \\
\mathrm{ppm}\end{array}$ & $\begin{array}{l}\mathrm{SO}_{4} \\
\mathrm{ppm}\end{array}$ & $\begin{array}{c}\mathrm{Li} \\
\mathrm{ppb}\end{array}$ & $\begin{array}{c}\mathrm{Be} \\
\mathrm{ppb}\end{array}$ & $\begin{array}{c}\mathrm{V} \\
\mathrm{ppb}\end{array}$ & $\begin{array}{c}\mathrm{Cr} \\
\mathrm{ppb}\end{array}$ & $\begin{array}{c}\mathrm{Co} \\
\mathrm{ppb}\end{array}$ & $\begin{array}{c}\mathrm{Ni} \\
\mathrm{ppb}\end{array}$ \\
\hline 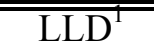 & 0.5 & $\overline{2}$ & $\overline{0.1}$ & 20 & 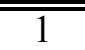 & $\overline{0.5}$ & $\overline{11}$ & 10 & 0.2 & 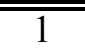 \\
\hline CS-1 & 0.7 & 32 & 3.7 & $<20$ & 37 & 0.5 & 106 & $<10$ & 2.1 & 20 \\
\hline $\mathrm{CS}-2$ & $<0.5$ & 29 & 3.4 & $<20$ & 24 & 1.0 & 55 & $<10$ & 1.7 & 17 \\
\hline CS-3 & 0.8 & 11 & 5.1 & $<20$ & 21 & 2.0 & 17 & $<10$ & 3.9 & 28 \\
\hline CS-4 & $<0.5$ & 38 & 2.6 & $<20$ & 40 & 0.5 & 97 & $<10$ & 1.1 & 18 \\
\hline CS-5 & $<0.5$ & 68 & 1.0 & $<20$ & 23 & $<0.5$ & 248 & $<10$ & 1.0 & 21 \\
\hline CS-6 & 0.8 & 6 & 1.1 & $<20$ & 2 & 0.6 & 5 & $<10$ & 0.9 & 11 \\
\hline CS-7 & 1.1 & 10 & 2.6 & $<20$ & 9 & 0.8 & 12 & $<10$ & 1.5 & 9 \\
\hline CS-8 & 4.7 & 20 & 19 & 37 & 31 & 1.2 & 128 & 131 & 5.4 & 206 \\
\hline CS-9 & 2.5 & 15 & 14 & 24 & 26 & 1.6 & 90 & 44 & 7.4 & 181 \\
\hline CS-10 & $<0.5$ & 18 & 3.7 & 98 & 55 & $<0.5$ & 40 & $<10$ & 5.3 & 39 \\
\hline CS-11 & $<0.5$ & 14 & 1.0 & 15100 & 84 & $<0.5$ & 7 & $<10$ & 11.4 & 286 \\
\hline CS-12 & 1.7 & 9 & 0.7 & 30 & $<1$ & $<0.5$ & 10 & $<10$ & 1.9 & 9 \\
\hline CS-13 & 2.9 & 11 & 0.8 & $<20$ & $<1$ & 0.6 & 10 & $<10$ & 2.9 & 7 \\
\hline CS-14 & 1.0 & 5 & 0.9 & 19700 & 147 & $<.5$ & 3 & $<10$ & 16.0 & 322 \\
\hline CS-15 & 1.4 & 4 & 0.6 & 13800 & 20 & $<.5$ & 13 & $<10$ & 8.7 & 246 \\
\hline CS-16 & $<0.5$ & 8 & 2.0 & 263 & 12 & 1.2 & 11 & $<10$ & 1.7 & 23 \\
\hline CS-17 & 0.8 & 3 & 1.4 & $<20$ & $<1$ & 0.6 & 8 & $<10$ & 1.4 & 11 \\
\hline CS-18 & 1.6 & 13 & 4.5 & $<20$ & 10 & $<.5$ & 32 & $<10$ & 3.0 & 9 \\
\hline CS-19 & 2.6 & 14 & 7.0 & $<20$ & 3 & 1.8 & 30 & $<10$ & 4.6 & 10 \\
\hline S01 & 1.3 & 49 & 7.0 & 24 & 17 & 0.5 & 190 & $<10$ & 3.4 & 26 \\
\hline S02 & 0.7 & 34 & 6.6 & 30 & 8 & $<0.5$ & 106 & $<10$ & 3.3 & 30 \\
\hline S03 & 1.7 & 24 & 3.2 & $<20$ & $<1$ & $<0.5$ & 41 & $<10$ & 3.7 & 30 \\
\hline S04 & 2.8 & 28 & 8.1 & 24 & 13 & 1.0 & 80 & $<10$ & 8.3 & 43 \\
\hline S05 & 1.3 & 40 & 5.8 & 21 & 13 & 0.9 & 49 & $<10$ & 2.9 & 25 \\
\hline S06 & 1.7 & 33 & 4.7 & $<20$ & 9 & 0.7 & 94 & $<10$ & 3.8 & 26 \\
\hline S07 & 1.4 & 41 & 8.1 & $<20$ & 23 & 0.7 & 128 & $<10$ & 4.2 & 24 \\
\hline S08 & 1.2 & 42 & 2.8 & $<20$ & 15 & 0.5 & 126 & $<10$ & 3.0 & 24 \\
\hline S09 & 2.0 & 70 & 3.3 & 52 & 74 & 0.6 & 753 & $<10$ & 5.1 & 28 \\
\hline $\mathrm{S} 10$ & 1.7 & 45 & 3.7 & 34 & 24 & $<.5$ & 181 & $<10$ & 2.7 & 20 \\
\hline S11 & 1.4 & 53 & 4.0 & $<20$ & 26 & 1.1 & 194 & $<10$ & 4.0 & 25 \\
\hline S12 & 0.8 & 37 & 15.2 & 34 & 8 & $<0.5$ & 100 & $<10$ & 7.4 & 45 \\
\hline S13 & $<0.5$ & 41 & 3.9 & 32 & 26 & $<0.5$ & 91 & $<10$ & 2.7 & 30 \\
\hline S14 & $<0.5$ & 16 & 5.4 & 23 & 7 & $<0.5$ & 53 & $<10$ & 1.4 & 32 \\
\hline S15 & 0.6 & 24 & 9.8 & 25 & 15 & $<0.5$ & 70 & $<10$ & 2.5 & 42 \\
\hline S16 & $<0.5$ & 29 & 6.2 & 30 & 12 & 0.5 & 45 & $<10$ & 2.3 & 44 \\
\hline S17 & $<0.5$ & 36 & 5.1 & 40 & 9 & 0.6 & 57 & $<10$ & 2.1 & 29 \\
\hline S01-R ${ }^{2}$ & $<0.5$ & 45 & 7.0 & 32 & 19 & $<0.5$ & 185 & $<10$ & 2.1 & 17 \\
\hline $\mathrm{CS}-1 \mathrm{R}^{2}$ & $<0.5$ & 30 & 3.9 & $<20$ & 36 & 0.6 & 94 & $<10$ & 1.7 & 30 \\
\hline
\end{tabular}

${ }^{1}$ lower limit of determinatin

${ }^{2}$ duplicate 
Table 7. Chemical analyses of water-soluble extractions of soils from the western United States

\begin{tabular}{|c|c|c|c|c|c|c|c|c|c|c|c|}
\hline Site No. & $\begin{array}{r}\mathrm{Cu} \\
\mathrm{ppb}\end{array}$ & $\begin{array}{r}\mathrm{Zn} \\
\mathrm{ppb}\end{array}$ & $\begin{array}{c}\mathrm{As} \\
\mathrm{ppb}\end{array}$ & $\begin{array}{c}\mathrm{Se} \\
\mathrm{ppb}\end{array}$ & $\begin{array}{l}\text { Mo } \\
\mathrm{ppb} \\
\end{array}$ & $\begin{array}{r}\mathrm{Cd} \\
\mathrm{ppb}\end{array}$ & $\begin{array}{r}\mathrm{Sb} \\
\mathrm{ppb} \\
\end{array}$ & $\begin{array}{c}\mathrm{Ba} \\
\mathrm{ppb}\end{array}$ & $\begin{array}{c}\mathrm{Pb} \\
\mathrm{ppb}\end{array}$ & $\begin{array}{l}\text { Th } \\
\mathrm{ppb}\end{array}$ & $\begin{array}{c}\mathrm{U} \\
\mathrm{ppb} \\
\end{array}$ \\
\hline$\overline{\text { LLD }}^{\top}$ & 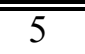 & 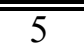 & 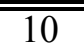 & $\overline{c 10}$ & 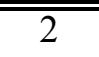 & 0.2 & $\overline{1}$ & 1 & 0.5 & 0.05 & 0.05 \\
\hline CS-1 & 80 & 14 & $<10$ & $<10$ & 2 & 0.5 & 2 & 403 & 13.8 & 9.2 & 0.22 \\
\hline $\mathrm{CS}-2$ & 73 & 19 & $<10$ & $<10$ & 2 & 0.4 & 2 & 359 & 11.5 & 6.1 & 0.16 \\
\hline $\mathrm{CS}-3$ & 71 & 49 & 10 & $<10$ & 6 & 1.1 & $<1$ & 234 & 9.2 & 11 & 0.52 \\
\hline CS-4 & 66 & 15 & 17 & $<10$ & 2 & 0.3 & 2 & 136 & 5.9 & 3.5 & 0.44 \\
\hline CS-5 & 47 & 8 & 37 & $<10$ & 3 & $<.2$ & 2 & 776 & 1.5 & 4.3 & 6.8 \\
\hline CS-6 & 26 & 13 & 13 & $<10$ & 7 & 0.3 & $<1$ & 89 & 6.3 & 3.0 & 8.8 \\
\hline CS-7 & 67 & 35 & 13 & $<10$ & 6 & 0.6 & $<1$ & 83 & 6.7 & 4.1 & 1.2 \\
\hline CS-8 & 243 & 654 & 25 & 21 & 31 & 9.5 & 4 & 291 & 564 & 17 & 5.4 \\
\hline CS-9 & 195 & 231 & 18 & 13 & 17 & 8.0 & 1 & 390 & 106 & 6.1 & 1.9 \\
\hline CS-10 & 88 & 15 & 12 & 11 & 3 & 0.4 & $<1$ & 206 & 2.7 & 3.0 & 0.72 \\
\hline CS-11 & 73 & 38 & $<10$ & 126 & 27 & 0.8 & $<1$ & 403 & 1.6 & 3.9 & 12 \\
\hline CS-12 & 28 & 11 & $<10$ & $<10$ & $<2$ & 0.3 & $<1$ & 310 & 6.2 & 0.60 & 0.37 \\
\hline CS-13 & 30 & 12 & $<10$ & $<10$ & $<2$ & 0.2 & $<1$ & 321 & 3.2 & 0.97 & 0.74 \\
\hline CS-14 & 58 & 28 & 28 & 307 & 86 & 0.3 & $<1$ & 345 & 1.2 & 5.8 & 0.97 \\
\hline CS-15 & 48 & 25 & 27 & 627 & 2520 & 5.3 & $<1$ & 105 & 1.0 & 3.2 & 17 \\
\hline CS-16 & 54 & 18 & $<10$ & 16 & 33 & 0.5 & $<1$ & 1230 & 2.9 & 0.96 & 0.56 \\
\hline CS-17 & 67 & 12 & $<10$ & $<10$ & 9 & 0.3 & $<1$ & 237 & 3.0 & 0.95 & 2.5 \\
\hline CS-18 & 95 & 32 & 14 & $<10$ & 12 & 1.5 & 3 & 111 & 26 & 0.70 & 1.1 \\
\hline CS-19 & 149 & 50 & 25 & $<10$ & 9 & 2.9 & 2 & 84 & 48 & 1.2 & 1.7 \\
\hline S01 & 112 & 32 & 25 & $<10$ & 4 & 0.6 & 4 & 160 & 15 & 0.98 & 0.68 \\
\hline $\mathrm{S} 02$ & 96 & 27 & 16 & 11 & 3 & 0.5 & 5 & 199 & 5.4 & 0.75 & 0.61 \\
\hline S03 & 49 & 24 & 11 & $<10$ & $<2$ & 0.3 & 30 & 37 & 7.9 & 0.41 & 0.19 \\
\hline S04 & 152 & 62 & 22 & 13 & 7 & 1.6 & 4 & 256 & 15 & 1.8 & 0.89 \\
\hline S05 & 75 & 31 & 10 & $<10$ & $<2$ & 0.6 & 3 & 152 & 13 & 0.82 & 0.44 \\
\hline S06 & 87 & 27 & 14 & $<10$ & $<2$ & 0.6 & 1 & 158 & 12 & 0.56 & 0.44 \\
\hline S07 & 89 & 19 & 16 & $<10$ & 3 & 0.6 & 2 & 151 & 7.7 & 0.80 & 0.73 \\
\hline S08 & 84 & 16 & 38 & 13 & $<2$ & 0.3 & 5 & 295 & 3.2 & 0.46 & 0.75 \\
\hline S09 & 67 & 19 & 85 & 11 & 3 & 0.4 & 2 & 781 & 4.8 & 1.3 & 5.8 \\
\hline S10 & 57 & 17 & 60 & $<10$ & 5 & 0.2 & 3 & 74 & 7.1 & 0.51 & 2.6 \\
\hline S11 & 129 & 15 & 26 & $<10$ & 2 & 1.9 & 24 & 178 & 4.4 & 0.58 & 0.86 \\
\hline S12 & 111 & 34 & 10 & 15 & 4 & 1.4 & 1 & 237 & 6.4 & 1.9 & 0.19 \\
\hline S13 & 77 & 14 & 30 & 18 & 3 & 0.4 & 2 & 361 & 2.2 & 0.48 & 0.57 \\
\hline S14 & 58 & 8 & $<10$ & $<10$ & $<2$ & $<.2$ & $<1$ & 260 & 1.6 & 0.40 & 0.34 \\
\hline S15 & 79 & 15 & 11 & $<10$ & 3 & 0.4 & $<1$ & 167 & 2.6 & 0.40 & 0.34 \\
\hline S16 & 108 & 20 & 12 & 14 & 4 & 0.8 & $<1$ & 229 & 2.5 & 0.70 & 0.39 \\
\hline $\mathrm{S} 17$ & 84 & 26 & 18 & $<10$ & 2 & 0.6 & 1 & 179 & 3.1 & 0.38 & 0.25 \\
\hline $\mathrm{S} 01-\mathrm{R}^{2}$ & 133 & 23 & 24 & $<10$ & 3 & 0.5 & 4 & 109 & 7.0 & 0.65 & 0.47 \\
\hline $\mathrm{CS}-1 \mathrm{R}^{2}$ & 82 & 16 & $<10$ & $<10$ & $<2$ & 0.5 & 1 & 397 & 9.0 & 1.4 & 0.25 \\
\hline
\end{tabular}

${ }^{1}$ lower limit of determinatin

${ }^{2}$ duplicate 
Table 8. Chemical analyses of sequential extractions of soils from Guam and Colorado

\begin{tabular}{|c|c|c|c|c|c|c|c|c|c|}
\hline \multirow[b]{2}{*}{ Site } & \multicolumn{6}{|c|}{ Fraction } & \multirow{2}{*}{$\begin{array}{l}\text { Total of } \\
\text { Fractions }\end{array}$} & \multirow[t]{2}{*}{ Total $^{1}$} & \multirow{2}{*}{$\begin{array}{c}\text { Percent } \\
\text { Rcovery }\end{array}$} \\
\hline & $A$ & B & C & $D$ & $E$ & $\mathrm{~F}$ & & & \\
\hline \multicolumn{10}{|c|}{ Al ppm } \\
\hline \multicolumn{10}{|c|}{ Guam } \\
\hline GS-01 & $<8$ & 86 & 18 & 1490 & 8110 & 50400 & 60104 & 84000 & $72 \%$ \\
\hline GS-04 & $<8$ & 63 & 10 & 1720 & 7000 & 56700 & 65493 & 80000 & $82 \%$ \\
\hline GS-08 & $<8$ & 76 & 13 & 1580 & 9210 & 47000 & 57879 & 66000 & $88 \%$ \\
\hline GS-13 & $<8$ & 69 & 13 & 1630 & 8920 & 44100 & 54732 & 63000 & $87 \%$ \\
\hline GS-18 & $<8$ & 234 & 121 & 5990 & 178000 & 21200 & 205545 & 260000 & $79 \%$ \\
\hline GS-22 & $<8$ & 563 & 75 & 2470 & 29200 & 82400 & 114708 & 140000 & $82 \%$ \\
\hline GS-24 & $<8$ & 101 & $<8$ & 6280 & 28800 & 31700 & 66881 & 73000 & $92 \%$ \\
\hline \multicolumn{10}{|c|}{ Colorado } \\
\hline CS-06 & $<8$ & 39 & 11 & 1480 & 6240 & 39900 & 47670 & 62000 & $77 \%$ \\
\hline CS-10 & $<8$ & 40 & 11 & 1020 & 5650 & 40300 & 47021 & 56000 & $84 \%$ \\
\hline CS-12 & $<8$ & 106 & 14 & 1780 & 12100 & 45000 & 59000 & 65000 & $91 \%$ \\
\hline CS-15 & $<8$ & 16 & $<8$ & 227 & 7500 & 16400 & 24143 & 23000 & $105 \%$ \\
\hline GS-22R ${ }^{2}$ & $<8$ & 591 & 80 & 2140 & 30500 & 102000 & 135311 & 140000 & $97 \%$ \\
\hline \multicolumn{10}{|c|}{ Ca ppm } \\
\hline \multicolumn{10}{|c|}{ Guam } \\
\hline GS-01 & 2730 & 999 & 386 & 586 & 190 & 10800 & 15691 & 20000 & $78 \%$ \\
\hline GS-04 & 4950 & 1400 & 96.8 & 700 & 289 & 14900 & 22336 & 26000 & $86 \%$ \\
\hline GS-08 & 8320 & 53900 & 1330 & 634 & 780 & 15300 & 80264 & 90000 & $89 \%$ \\
\hline GS-13 & 6410 & 52900 & 1620 & 1660 & 205 & 8980 & 71775 & 82000 & $88 \%$ \\
\hline GS-18 & 1570 & 1490 & 262 & 181 & 4350 & 1120 & 8973 & 12000 & $75 \%$ \\
\hline GS-22 & 2070 & $<20$ & $<20$ & $<20$ & $<20$ & $<20$ & 2070 & 2000 & $104 \%$ \\
\hline GS-24 & 6400 & 1040 & 66.3 & 282 & 786 & 9690 & 18264 & 23000 & $79 \%$ \\
\hline \multicolumn{10}{|c|}{ Colorado } \\
\hline CS-06 & 1530 & 22.7 & 29.8 & 241 & $<20$ & 682 & 2506 & 4000 & $63 \%$ \\
\hline CS-10 & 5640 & 14600 & 3250 & 16700 & 387 & 345 & 40922 & 40000 & $102 \%$ \\
\hline CS-12 & 941 & $<20$ & 30.2 & 193 & 296 & 7140 & 8600 & 11000 & $78 \%$ \\
\hline CS-15 & 59500 & 34600 & 9400 & 17400 & 338 & 287 & 121525 & 110000 & $110 \%$ \\
\hline GS-22R ${ }^{2}$ & 1710 & $<20$ & $<20$ & $<20$ & $<20$ & $<20$ & 1710 & 2000 & $86 \%$ \\
\hline \multicolumn{10}{|c|}{ Fe ppm } \\
\hline \multicolumn{10}{|c|}{ Guam } \\
\hline GS-01 & $<50$ & $<50$ & $<50$ & 716 & 18200 & 18200 & 37116 & 35000 & $106 \%$ \\
\hline GS-04 & $<50$ & $<50$ & $<50$ & 925 & 16100 & 22300 & 39325 & 36000 & $109 \%$ \\
\hline GS-08 & $<50$ & $<50$ & $<50$ & 1840 & 28000 & 18700 & 48540 & 47000 & $103 \%$ \\
\hline GS-13 & $<50$ & $<50$ & $<50$ & 448 & 17800 & 18800 & 37048 & 36000 & $103 \%$ \\
\hline GS-18 & $<50$ & $<50$ & 110 & 1890 & 111000 & 33900 & 146900 & 140000 & $105 \%$ \\
\hline GS-22 & $<50$ & $<50$ & 202 & 1060 & 116000 & 33700 & 150962 & 130000 & $116 \%$ \\
\hline GS-24 & $<50$ & $<50$ & 70 & 8600 & 56200 & 18300 & 83170 & 77000 & $108 \%$ \\
\hline \multicolumn{10}{|c|}{ Colorado } \\
\hline CS-06 & $<50$ & $<50$ & $<50$ & 2340 & 17900 & 9760 & 30000 & 27000 & $111 \%$ \\
\hline CS-10 & $<50$ & $<50$ & $<50$ & 638 & 18200 & 7700 & 26538 & 24000 & $111 \%$ \\
\hline CS-12 & $<50$ & $<50$ & $<50$ & 4050 & 26400 & 13300 & 43750 & 38000 & $115 \%$ \\
\hline CS-15 & $<50$ & $<50$ & 338 & 907 & 9780 & 1120 & 12145 & 9700 & $125 \%$ \\
\hline GS-22R ${ }^{2}$ & $<50$ & $<50$ & 203 & 902 & 112000 & 34100 & 147205 & 130000 & $113 \%$ \\
\hline \multicolumn{10}{|c|}{ Mg ppm } \\
\hline
\end{tabular}


Table 8. Chemical analyses of sequential extractions of soils from Guam and Colorado

\begin{tabular}{|c|c|c|c|c|c|c|c|c|c|}
\hline \multirow[b]{2}{*}{ Site } & \multicolumn{6}{|c|}{ Fraction } & \multirow{2}{*}{$\begin{array}{l}\text { Total of } \\
\text { Fractions }\end{array}$} & \multirow[t]{2}{*}{ Total $^{1}$} & \multirow{2}{*}{$\begin{array}{c}\text { Percent } \\
\text { Rcovery }\end{array}$} \\
\hline & $A$ & B & C & $D$ & $E$ & $\mathrm{~F}$ & & & \\
\hline \multicolumn{10}{|c|}{ Guam } \\
\hline GS-01 & 209 & 109 & 22 & 422 & 2590 & 3260 & 6612 & 8200 & $81 \%$ \\
\hline GS-04 & 333 & 104 & 16 & 353 & 2370 & 4210 & 7386 & 8800 & $84 \%$ \\
\hline GS-08 & 251 & 390 & 35 & 1270 & 8990 & 5130 & 16066 & 17000 & $95 \%$ \\
\hline GS-13 & 218 & 424 & 39 & 571 & 3350 & 4460 & 9062 & 11000 & $82 \%$ \\
\hline GS-18 & 19 & 60 & 38 & 60 & 379 & 201 & 757 & 1000 & $76 \%$ \\
\hline GS-22 & 1140 & 74 & 7 & 42 & 2170 & 4730 & 8163 & 11000 & $74 \%$ \\
\hline GS-24 & 2700 & 292 & 20 & 2910 & 18700 & 9000 & 33622 & 37000 & $91 \%$ \\
\hline \multicolumn{10}{|c|}{ Colorado } \\
\hline CS-06 & 112 & 11 & 3 & 89 & 1920 & 2000 & 4135 & 5300 & $78 \%$ \\
\hline CS-10 & 306 & 2120 & 1720 & 11000 & 2420 & 2510 & 20076 & 20000 & $100 \%$ \\
\hline CS-12 & 262 & 22 & 4 & 606 & 4750 & 4100 & 9744 & 12000 & $81 \%$ \\
\hline CS-15 & 734 & 3600 & 6530 & 11700 & 15800 & 919 & 39283 & 32000 & $123 \%$ \\
\hline GS-22R ${ }^{2}$ & 1120 & 84 & 8 & 39 & 2250 & 5090 & 8591 & 11000 & $78 \%$ \\
\hline \multicolumn{10}{|c|}{ Mn ppm } \\
\hline \multicolumn{10}{|c|}{ Guam } \\
\hline GS-01 & 0.5 & 38 & 674 & 168 & 167 & 218 & 1265 & 1300 & $97 \%$ \\
\hline GS-04 & 4 & 66 & 234 & 106 & 99 & 224 & 733 & 820 & $89 \%$ \\
\hline GS-08 & $<0.2$ & 39 & 228 & 81 & 273 & 214 & 835 & 890 & $94 \%$ \\
\hline GS-13 & $<0.2$ & 46 & 276 & 71 & 100 & 203 & 696 & 780 & $89 \%$ \\
\hline GS-18 & $<0.2$ & 104 & 4650 & 456 & 510 & 95 & 5815 & 5600 & $104 \%$ \\
\hline GS-22 & 52 & 69 & 973 & 723 & 575 & 73 & 2465 & 2300 & $107 \%$ \\
\hline GS-24 & 33 & 111 & 364 & 392 & 430 & 123 & 1453 & 1400 & $104 \%$ \\
\hline \multicolumn{10}{|c|}{ Colorado } \\
\hline CS-06 & 1.2 & 11 & 352 & 589 & 343 & 92 & 1388 & 1200 & $116 \%$ \\
\hline CS-10 & 0.2 & 41 & 23 & 25 & 57 & 29 & 174 & 180 & $97 \%$ \\
\hline CS-12 & 4.9 & 11 & 53 & 114 & 311 & 140 & 634 & 610 & $104 \%$ \\
\hline CS-15 & 0.4 & 48 & 33 & 52 & 39 & 11 & 183 & 180 & $102 \%$ \\
\hline GS-22R ${ }^{2}$ & 52 & 73 & 942 & 634 & 575 & 76 & 2352 & 2300 & $102 \%$ \\
\hline \multicolumn{10}{|c|}{ K ppm } \\
\hline \multicolumn{10}{|c|}{ Guam } \\
\hline GS-01 & 1509 & 251 & 86 & 415 & 2813 & 27571 & 32645 & 30000 & $109 \%$ \\
\hline GS-04 & 915 & 188 & 62 & 309 & 2023 & 24870 & 28367 & 25000 & $113 \%$ \\
\hline GS-08 & 702 & 161 & 65 & 252 & 2285 & 14323 & 17788 & 16000 & $111 \%$ \\
\hline GS-13 & 571 & 144 & 61 & 234 & 2025 & 17764 & 20799 & 19000 & $109 \%$ \\
\hline GS-18 & 61 & 20 & 23 & 31 & 18 & 240 & 392 & 300 & $131 \%$ \\
\hline GS-22 & 433 & 31 & 34 & 51 & 1727 & 2882 & 5158 & 4000 & $129 \%$ \\
\hline GS-24 & 1057 & 185 & 58 & 289 & 2350 & 2949 & 6887 & 5500 & $125 \%$ \\
\hline \multicolumn{10}{|c|}{ Colorado } \\
\hline CS-06 & 320 & 94 & 32 & 73 & 1032 & 36929 & 38479 & 33000 & $117 \%$ \\
\hline CS-10 & 810 & 167 & 57 & 206 & 1404 & 18553 & 21197 & 18000 & $118 \%$ \\
\hline CS-12 & 387 & 98 & 32 & 32 & 973 & 18248 & 19770 & 17000 & $116 \%$ \\
\hline CS-15 & 328 & 142 & 33 & 21 & 679 & 17285 & 18488 & 15000 & $123 \%$ \\
\hline GS-22R ${ }^{2}$ & 431 & 30 & 33 & 12 & 1741 & 2809 & 5057 & 4000 & $126 \%$ \\
\hline \multicolumn{10}{|c|}{ As ppm } \\
\hline \multicolumn{10}{|c|}{ Guam } \\
\hline
\end{tabular}


Table 8. Chemical analyses of sequential extractions of soils from Guam and Colorado

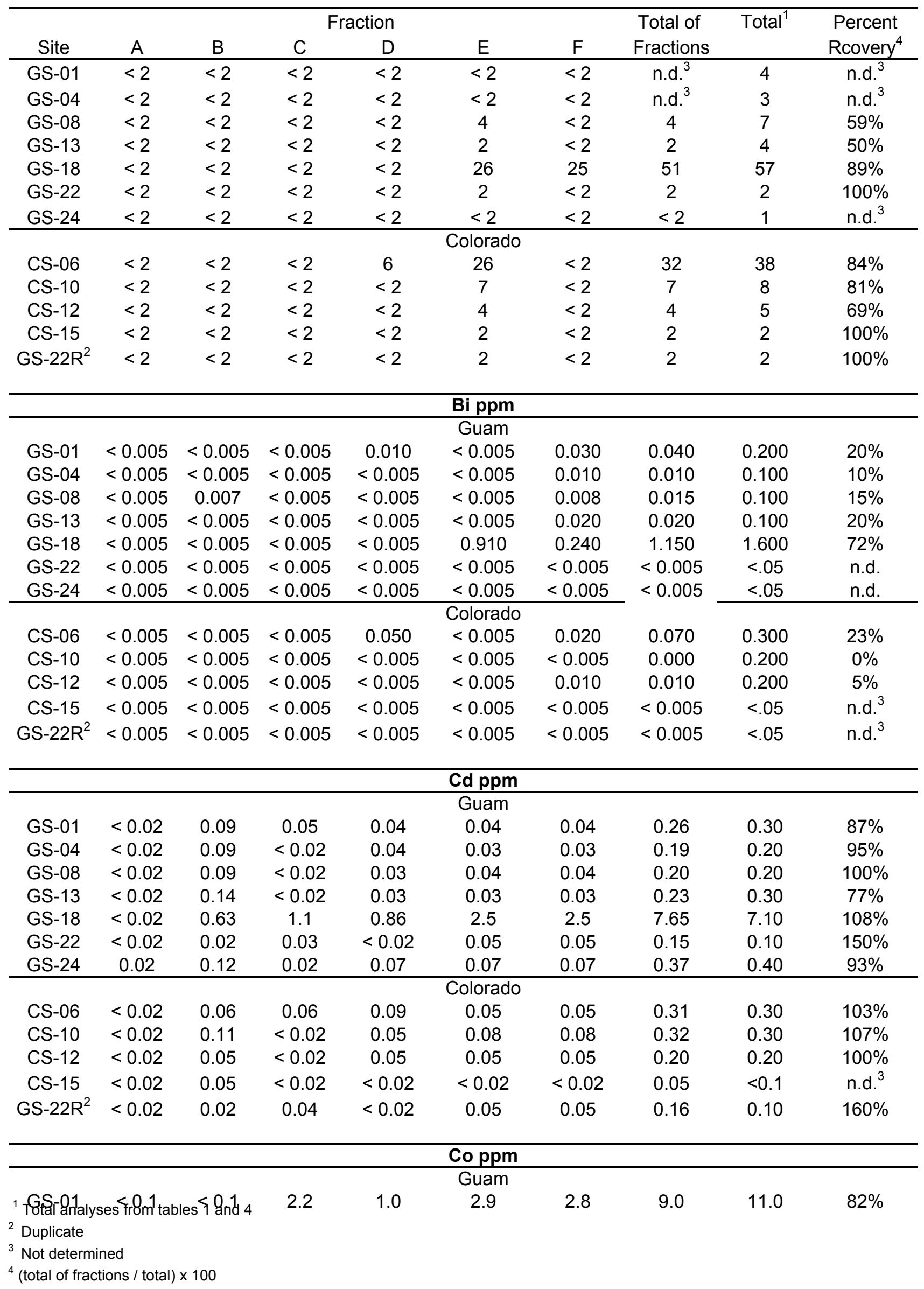


Table 8. Chemical analyses of sequential extractions of soils from Guam and Colorado

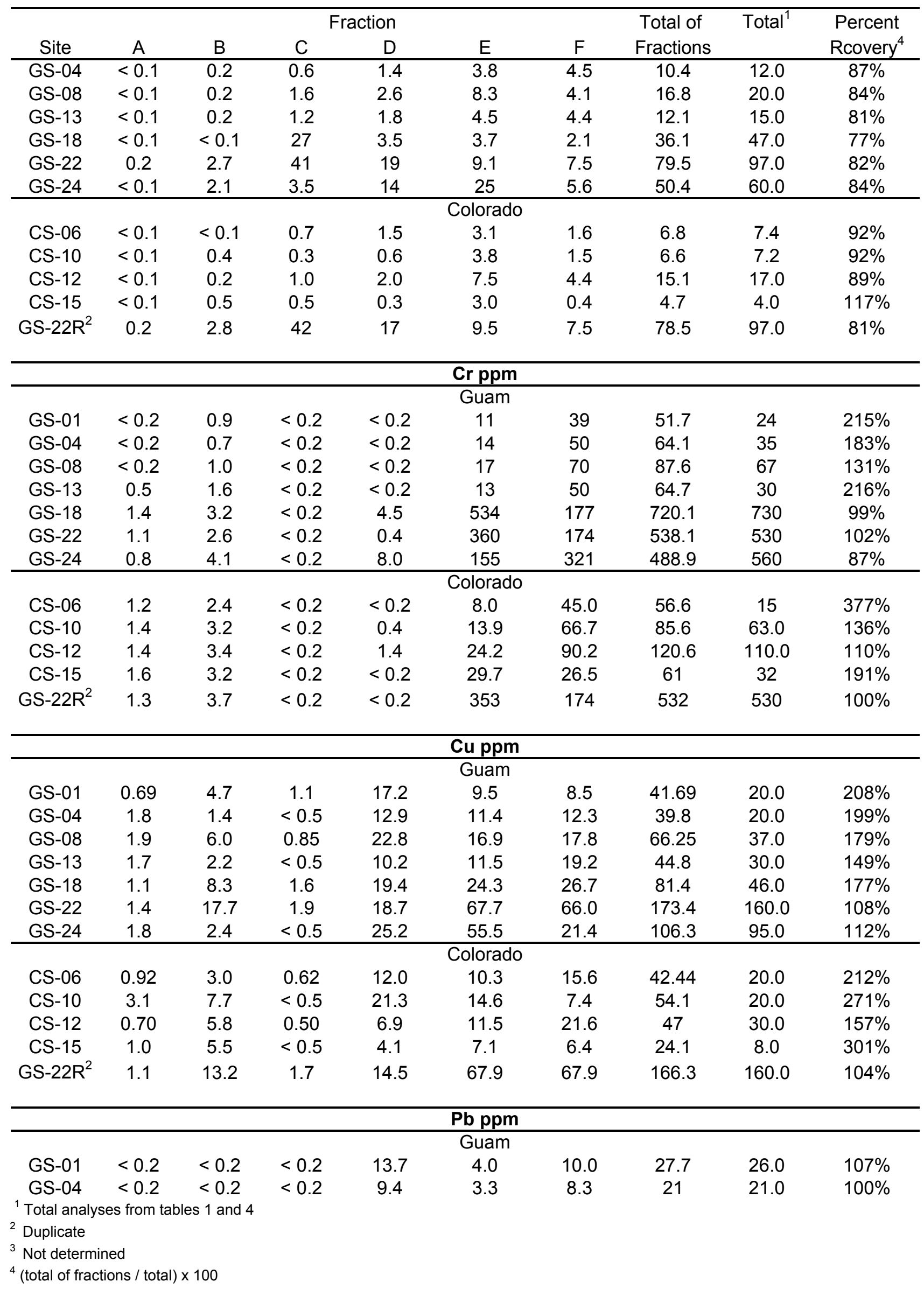


Table 8. Chemical analyses of sequential extractions of soils from Guam and Colorado

\begin{tabular}{|c|c|c|c|c|c|c|c|c|c|}
\hline \multirow[b]{2}{*}{ Site } & \multicolumn{6}{|c|}{ Fraction } & \multirow{2}{*}{$\begin{array}{l}\text { Total of } \\
\text { Fractions }\end{array}$} & \multirow[t]{2}{*}{ Total $^{1}$} & \multirow{2}{*}{$\begin{array}{c}\text { Percent } \\
\text { Rcovery }\end{array}$} \\
\hline & A & B & C & $D$ & $E$ & $\mathrm{~F}$ & & & \\
\hline GS-08 & $<0.2$ & $<0.2$ & $<0.2$ & 5.3 & 3.9 & 6.0 & 15.2 & 13.0 & $117 \%$ \\
\hline GS-13 & $<0.2$ & $<0.2$ & $<0.2$ & 6.2 & 3.8 & 9.1 & 19.1 & 16.0 & $119 \%$ \\
\hline GS-18 & $<0.2$ & $<0.2$ & $<0.2$ & 6.9 & 46.6 & 19.4 & 72.9 & 77.0 & $95 \%$ \\
\hline GS-22 & $<0.2$ & $<0.2$ & $<0.2$ & 1.5 & 2.0 & 1.1 & 4.6 & 2.0 & $230 \%$ \\
\hline GS-24 & $<0.2$ & $<0.2$ & $<0.2$ & 3.6 & 1.8 & 1.9 & 7.3 & 3.6 & $203 \%$ \\
\hline \multicolumn{10}{|c|}{ Colorado } \\
\hline CS-06 & $<0.2$ & $<0.2$ & $<0.2$ & 29.8 & 17.4 & 8.0 & 55.2 & 56.0 & $99 \%$ \\
\hline CS-10 & $<0.2$ & $<0.2$ & $<0.2$ & 6.3 & 8.9 & 4.1 & 19.3 & 20.0 & $97 \%$ \\
\hline CS-12 & $<0.2$ & $<0.2$ & $<0.2$ & 8.8 & 7.4 & 6.9 & 23.1 & 26.0 & $89 \%$ \\
\hline CS-15 & $<0.2$ & $<0.2$ & $<0.2$ & 0.79 & 1.1 & 5.3 & 7.19 & 4.5 & $160 \%$ \\
\hline GS-22R ${ }^{2}$ & $<0.2$ & $<0.2$ & $<0.2$ & 1.1 & 1.9 & 1.2 & 4.2 & 2.0 & $210 \%$ \\
\hline \multicolumn{10}{|c|}{ Mo ppm } \\
\hline \multicolumn{10}{|c|}{ Guam } \\
\hline GS-01 & $<0.2$ & $<0.2$ & $<0.2$ & $<0.2$ & 0.89 & 0.72 & 1.61 & 1.3 & $124 \%$ \\
\hline GS-04 & $<0.2$ & $<0.2$ & $<0.2$ & $<0.2$ & 0.63 & 0.80 & 1.43 & 1.1 & $130 \%$ \\
\hline GS-08 & $<0.2$ & $<0.2$ & $<0.2$ & $<0.2$ & 0.65 & 0.53 & 1.18 & 0.8 & $148 \%$ \\
\hline GS-13 & $<0.2$ & $<0.2$ & $<0.2$ & $<0.2$ & 0.62 & 1.14 & 1.76 & 0.8 & $220 \%$ \\
\hline GS-18 & $<0.2$ & $<0.2$ & $<0.2$ & $<0.2$ & 1.46 & 1.80 & 3.26 & 4.3 & $76 \%$ \\
\hline GS-22 & $<0.2$ & $<0.2$ & $<0.2$ & $<0.2$ & 0.73 & 0.32 & 1.05 & 0.6 & $175 \%$ \\
\hline GS-24 & $<0.2$ & $<0.2$ & $<0.2$ & $<0.2$ & 0.58 & 0.61 & 1.19 & 0.7 & $170 \%$ \\
\hline \multicolumn{10}{|c|}{ Colorado } \\
\hline CS-06 & $<0.2$ & $<0.2$ & $<0.2$ & 0.31 & 6.63 & 1.27 & 8.21 & 12.00 & $68 \%$ \\
\hline CS-10 & $<0.2$ & $<0.2$ & $<0.2$ & $<0.2$ & 1.36 & 0.68 & 2.04 & 1.8 & $113 \%$ \\
\hline CS -12 & $<0.2$ & $<0.2$ & $<0.2$ & $<0.2$ & 0.86 & 0.64 & 1.5 & 1.1 & $136 \%$ \\
\hline CS-15 & $<0.2$ & 0.25 & $<0.2$ & 0.40 & 4.74 & 0.66 & 6.05 & 9.50 & $64 \%$ \\
\hline GS-22R ${ }^{2}$ & $<0.2$ & $<0.2$ & $<0.2$ & $<0.2$ & 0.76 & 0.30 & 1.06 & 0.6 & $177 \%$ \\
\hline \multicolumn{10}{|c|}{ Ni ppm } \\
\hline \multicolumn{10}{|c|}{ Guam } \\
\hline GS-01 & $<1$ & 1.2 & $<1$ & 2.6 & 7.3 & 4.1 & 15.2 & 12.0 & $127 \%$ \\
\hline GS-04 & $<1$ & $<1$ & $<1$ & 2.6 & 8.2 & 5.9 & 16.7 & 14.0 & $119 \%$ \\
\hline GS-08 & $<1$ & 2.1 & $<1$ & 5.8 & 23.3 & 7.3 & 38.5 & 39.0 & $99 \%$ \\
\hline GS-13 & $<1$ & 1.6 & $<1$ & 3.0 & 10.8 & 7.8 & 23.2 & 21.0 & $110 \%$ \\
\hline GS-18 & $<1$ & 4.1 & 69.0 & 21.2 & 130 & 39.7 & 264 & 320.0 & $83 \%$ \\
\hline GS-22 & $<1$ & 1.8 & $<1$ & 3.9 & 64.7 & 272 & 342.4 & 380.0 & $90 \%$ \\
\hline GS-24 & $<1$ & 8.2 & $<1$ & 30.6 & 116 & 61.2 & 216 & 240.0 & $90 \%$ \\
\hline \multicolumn{10}{|c|}{ Colorado } \\
\hline CS-06 & $<1$ & $<1$ & $<1$ & 1.0 & 6.4 & 5.8 & 13.2 & 6.6 & $200 \%$ \\
\hline CS-10 & $<1$ & 1.1 & $<1$ & 2.9 & 16.1 & 9.1 & 29.2 & 23.0 & $127 \%$ \\
\hline CS-12 & $<1$ & $<1$ & $<1$ & 2.3 & 28.6 & 20.0 & 50.9 & 47.0 & $108 \%$ \\
\hline CS -15 & $<1$ & 1.0 & $<1$ & $<1$ & 16.5 & 6.0 & 23.5 & 13.0 & $181 \%$ \\
\hline GS-22R ${ }^{2}$ & $<1$ & 1.2 & $<1$ & 2.7 & 67.3 & 272 & 343.2 & 380.0 & $90 \%$ \\
\hline \multicolumn{10}{|c|}{ Sr ppm } \\
\hline \multicolumn{10}{|c|}{ Guam } \\
\hline GS-01 & 17.0 & 5.02 & 0.81 & 2.70 & 9.44 & 346 & 380.97 & 500 & $76 \%$ \\
\hline GS-04 & 21.2 & 5.27 & 0.42 & 2.44 & 7.21 & 384 & 420.54 & 490 & $86 \%$ \\
\hline GS-08 & 24.3 & 53.1 & 1.61 & 1.97 & 9.48 & 198 & 288.46 & 350 & $82 \%$ \\
\hline \multicolumn{10}{|c|}{1 Total analyses from tables 1 and 4} \\
\hline Duplicate & & & & & & & & & \\
\hline Not determi & & & & & & & & & \\
\hline
\end{tabular}


Table 8. Chemical analyses of sequential extractions of soils from Guam and Colorado

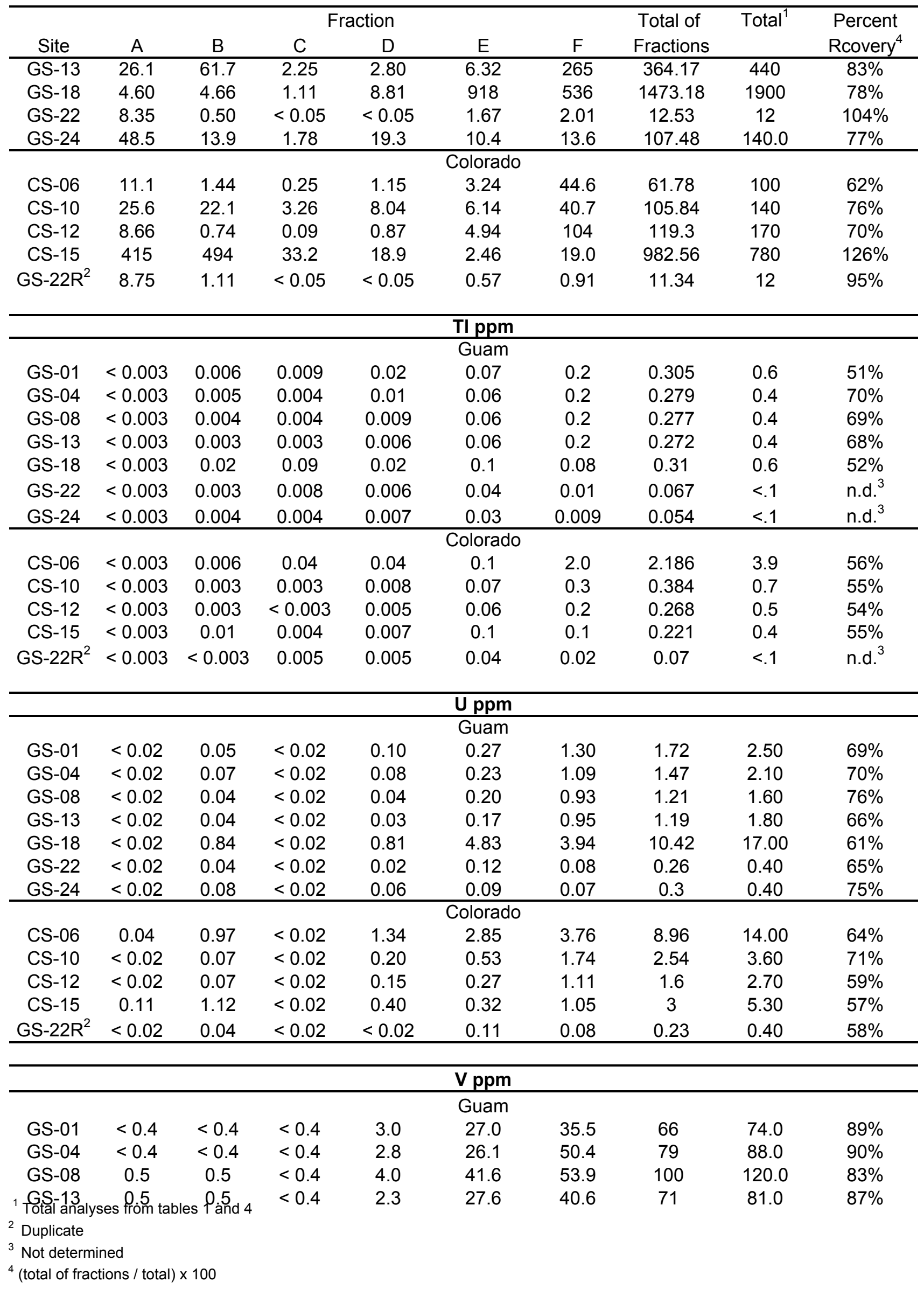


Table 8. Chemical analyses of sequential extractions of soils from Guam and Colorado

\begin{tabular}{|c|c|c|c|c|c|c|c|c|c|}
\hline \multirow[b]{2}{*}{ Site } & \multicolumn{6}{|c|}{ Fraction } & \multirow{2}{*}{$\begin{array}{l}\text { Total of } \\
\text { Fractions }\end{array}$} & \multirow[t]{2}{*}{ Total $^{1}$} & \multirow{2}{*}{$\begin{array}{c}\text { Percent } \\
\text { Rcovery }\end{array}$} \\
\hline & A & $B$ & C & D & $E$ & $\mathrm{~F}$ & & & \\
\hline GS-18 & $<0.4$ & 0.6 & 10.8 & 37.5 & 405 & 110 & 553 & 510.0 & $108 \%$ \\
\hline GS-22 & $<0.4$ & 0.6 & $<0.4$ & 5.3 & 361 & 25.7 & 392 & 280.0 & $140 \%$ \\
\hline GS-24 & $<0.4$ & 0.8 & $<0.4$ & 27.4 & 106 & 37.8 & 171 & 190.0 & $90 \%$ \\
\hline \multicolumn{10}{|c|}{ Colorado } \\
\hline CS-06 & $<0.4$ & 0.6 & $<0.4$ & 1.9 & 11.0 & 33.6 & 47 & 49.0 & $95 \%$ \\
\hline CS-10 & 0.6 & 1.0 & $<0.4$ & 2.6 & 20.1 & 65.2 & 88 & 95.0 & $93 \%$ \\
\hline CS-12 & 0.4 & 0.8 & $<0.4$ & 6.8 & 38.1 & 46.1 & 91 & 96.0 & $95 \%$ \\
\hline CS-15 & 0.5 & 1 & $<0.4$ & 1.0 & 22.8 & 10.1 & 34 & 32.0 & $106 \%$ \\
\hline GS-22R ${ }^{2}$ & $<0.4$ & 0.8 & $<0.4$ & 4.7 & 350 & 23.8 & 379 & 280.0 & $135 \%$ \\
\hline \multicolumn{10}{|c|}{ Zn ppm } \\
\hline \multicolumn{10}{|c|}{ Guam } \\
\hline GS-01 & $<5$ & $<5$ & $<5$ & 9.0 & 44.1 & 44.5 & 97.6 & 110.0 & $89 \%$ \\
\hline GS-04 & $<5$ & $<5$ & $<5$ & 8.1 & 29.6 & 40.3 & 78 & 81.0 & $96 \%$ \\
\hline GS-08 & $<5$ & $<5$ & $<5$ & 8.5 & 42.3 & 35.9 & 86.7 & 86.0 & $101 \%$ \\
\hline GS-13 & $<5$ & $<5$ & $<5$ & 7.2 & 34.4 & 35.3 & 76.9 & 79.0 & $97 \%$ \\
\hline GS-18 & $<5$ & $<5$ & $<5$ & $<5$ & 27.5 & 28.3 & 55.8 & 57.0 & $98 \%$ \\
\hline GS-22 & $<5$ & $<5$ & $<5$ & $<5$ & 54.8 & 34.3 & 89.1 & 97.0 & $92 \%$ \\
\hline GS-24 & $<5$ & $<5$ & $<5$ & 19.9 & 50.0 & 14.9 & 84.8 & 92.0 & $92 \%$ \\
\hline \multicolumn{10}{|c|}{ Colorado } \\
\hline CS-06 & $<5$ & $<5$ & $<5$ & 9.8 & 67.9 & 44.9 & 122.6 & 130.0 & $94 \%$ \\
\hline CS-10 & $<5$ & $<5$ & $<5$ & 6.9 & 56.6 & 25.2 & 88.7 & 93.0 & $95 \%$ \\
\hline CS-12 & $<5$ & $<5$ & $<5$ & $<5$ & 44.2 & 40.3 & 84.5 & 87.0 & $97 \%$ \\
\hline CS-15 & $<5$ & $<5$ & $<5$ & $<5$ & 12.2 & $<5$ & 12.2 & 10.0 & $122 \%$ \\
\hline GS-22R ${ }^{2}$ & $<5$ & $<5$ & $<5$ & $<5$ & 55.3 & 33.9 & 89.2 & 97.0 & $92 \%$ \\
\hline
\end{tabular}

\footnotetext{
${ }^{1}$ Total analyses from tables 1 and 4

2 Duplicate

${ }^{3}$ Not determined

${ }^{4}$ (total of fractions / total) $\times 100$
} 
Table 9. Chemical analyses of simulated lung fluid dissolution of soils from Guam and Colorado.

\begin{tabular}{|c|c|c|c|c|c|c|c|}
\hline Field No & Location & Rock type of formation & $\mathrm{Al} \mathrm{ppm}$ & Ca ppm & $\mathrm{Mg} \mathrm{ppm}$ & $\mathrm{K} \mathrm{ppm}$ & $\mathrm{Mn} \mathrm{ppb}$ \\
\hline $\operatorname{LLD}^{1}$ & & & 0.02 & 10 & 2.0 & 6.0 & 10 \\
\hline \multicolumn{8}{|l|}{ Guam } \\
\hline GS-4L & Umatac & Facpi Fm & 1.6 & 2230 & 262 & 468 & 8770 \\
\hline GS-4LD & duplicate & & 1.2 & 2350 & 243 & 516 & 9980 \\
\hline GS-8L & Merizo & Facpi Fm joint & $<0.02$ & 2280 & 215 & 374 & 2960 \\
\hline GS-10L & Inarajan & Bolanos Fm & $<0.02$ & 1770 & 175 & 616 & 2040 \\
\hline GS-13L & Umatac & Facpi Fm & 1.9 & 2480 & 176 & 215 & 3430 \\
\hline GS-18L & Northern Guam & limestone & 1.7 & 480 & 12 & 6 & 50 \\
\hline GS-22L & Central Guam & Alutom Fm & $<0.02$ & 620 & 758 & 146 & 18500 \\
\hline GS-24L & Sella Bay & Facpi Fm & 2.2 & 3230 & 1600 & 458 & 38900 \\
\hline \multicolumn{8}{|l|}{ Colorado } \\
\hline CS-6L & Lake City & mineralize tuff breccia & 0.8 & 1090 & 85 & 93 & 970 \\
\hline CS-12L & Grand Mesa & basalt & $<0.02$ & 790 & 205 & 129 & 620 \\
\hline CS-18L & So. Table Mtn. & basalt & 0.5 & 1096 & 203 & 227 & 2140 \\
\hline
\end{tabular}

${ }^{1}$ lower limit of determination 
Table 9. Chemical analyses of simulated lung fluid dissolution of soils from Guam and Colorado.

\begin{tabular}{|c|c|c|c|c|c|c|c|}
\hline Field No & Location & Rock type of formation & Fe ppm & Si ppm & $\mathrm{Ag} \mathrm{ppb}$ & $\mathrm{Ba} \mathrm{ppb}$ & Be ppb \\
\hline $\operatorname{LLD}^{1}$ & & & 10 & 40 & 600 & 20 & 10 \\
\hline \multicolumn{8}{|l|}{ Guam } \\
\hline GS-4L & Umatac & Facpi Fm & $<10$ & 262 & $<600$ & 7440 & $<10$ \\
\hline GS-4LD & duplicate & & $<10$ & 262 & $<600$ & 7360 & $<10$ \\
\hline GS-8L & Merizo & Facpi Fm joint & $<10$ & 31 & $<600$ & 11100 & $<10$ \\
\hline GS-10L & Inarajan & Bolanos Fm & $<10$ & 290 & $<600$ & 7810 & $<10$ \\
\hline GS-13L & Umatac & Facpi Fm & $<10$ & 312 & $<600$ & 17600 & $<10$ \\
\hline GS-18L & Northern Guam & limestone & $<10$ & $<40$ & $<600$ & $<20$ & $<10$ \\
\hline GS-22L & Central Guam & Alutom Fm & $<10$ & $<40$ & $<600$ & 2800 & $<10$ \\
\hline GS-24L & Sella Bay & Facpi Fm & $<10$ & 245 & $<600$ & 700 & $<10$ \\
\hline \multicolumn{8}{|l|}{ Colorado } \\
\hline CS-6L & Lake City & mineralize tuff breccia & $<10$ & $<40$ & $<600$ & 2210 & $<10$ \\
\hline CS-12L & Grand Mesa & basalt & $<10$ & $<40$ & $<600$ & 14600 & $<10$ \\
\hline CS-18L & So. Table Mtn. & basalt & $<10$ & 86 & $<600$ & 8500 & $<10$ \\
\hline
\end{tabular}

${ }^{1}$ lower limit of determination 
Table 9. Chemical analyses of simulated lung fluid dissolution of soils from Guam and Colorado.

\begin{tabular}{lccccccc}
\hline Field No & Location & Rock type of formation & Bi ppb & Ce ppb & Co ppb & Cr ppb & Ga ppb \\
\hline LLD & & & & & & & \\
Guam & & 10 & 20 & 4 & 200 & 4 \\
GS-4L & Umatac & Facpi Fm & $<10$ & $<20$ & 54 & $<200$ & $<4$ \\
GS-4LD & duplicate & & & & & & \\
GS-8L & Merizo & Facpi Fm joint & $<10$ & $<20$ & 34 & $<200$ & $<4$ \\
GS-10L & Inarajan & Bolanos Fm & $<10$ & $<20$ & 22 & $<200$ & $<4$ \\
GS-13L & Umatac & Facpi Fm & $<10$ & $<20$ & 36 & $<200$ & $<4$ \\
GS-18L & Northern Guam & limestone & $<10$ & $<20$ & 17 & 1510 & $<4$ \\
GS-22L & Central Guam & Alutom Fm & $<10$ & $<20$ & 170 & $<200$ & $<4$ \\
GS-24L & Sella Bay & Facpi Fm & $<10$ & $<20$ & 255 & 280 & $<4$ \\
& & & & & & & \\
Colorado & & & & & & & \\
CS-6L & Lake City & mineralize tuff breccia & $<10$ & $<20$ & $<4$ & $<200$ & $<4$ \\
CS-12L & Grand Mesa & basalt & $<10$ & $<20$ & 6 & $<200$ & $<4$ \\
CS-18L & So. Table Mtn. & basalt & $<10$ & $<20$ & 7 & $<200$ & $<4$ \\
\hline
\end{tabular}

${ }^{1}$ lower limit of determination 
Table 9. Chemical analyses of simulated lung fluid dissolution of soils from Guam and Colorado.

\begin{tabular}{|c|c|c|c|c|c|c|c|}
\hline Field No & Location & Rock type of formation & Ge ppb & La ppb & Li ppb & $\mathrm{Ni} \mathrm{ppb}$ & $\mathrm{Pb} \mathrm{ppb}$ \\
\hline $\operatorname{LLD}^{1}$ & & & 4 & 20 & 20 & 20 & 10 \\
\hline \multicolumn{8}{|l|}{ Guam } \\
\hline GS-4L & Umatac & Facpi Fm & $<4$ & $<20$ & 152 & 124 & 13 \\
\hline GS-4LD & duplicate & & $<4$ & $<20$ & 127 & 102 & $<10$ \\
\hline GS-8L & Merizo & Facpi Fm joint & $<4$ & $<20$ & 23 & 45 & $<10$ \\
\hline GS-10L & Inarajan & Bolanos Fm & $<4$ & $<20$ & $<20$ & $<20$ & $<10$ \\
\hline GS-13L & Umatac & Facpi Fm & $<4$ & $<20$ & $<20$ & $<20$ & $<10$ \\
\hline GS-18L & Northern Guam & limestone & $<4$ & $<20$ & $<20$ & 133 & $<10$ \\
\hline GS-22L & Central Guam & Alutom Fm & $<4$ & $<20$ & $<20$ & 151 & $<10$ \\
\hline \multirow[t]{2}{*}{ GS-24L } & Sella Bay & Facpi Fm & $<4$ & $<20$ & $<20$ & 1070 & $<10$ \\
\hline & & & & & & & $<10$ \\
\hline Colorado & & & & & & & $<10$ \\
\hline CS-6L & Lake City & mineralize tuff breccia & $<4$ & $<20$ & $<20$ & $<20$ & $<10$ \\
\hline CS-12L & Grand Mesa & basalt & $<4$ & $<20$ & $<20$ & $<20$ & $<10$ \\
\hline CS-18L & So. Table Mtn. & basalt & $<4$ & $<20$ & $<20$ & $<20$ & $<10$ \\
\hline
\end{tabular}

${ }^{1}$ lower limit of determination 
Table 9. Chemical analyses of simulated lung fluid dissolution of soils from Guam and Colorado.

\begin{tabular}{|c|c|c|c|c|c|c|c|}
\hline Field No & Location & Rock type of formation & $\mathrm{Rb} p p b$ & Sc ppb & Sr ppb & $\mathrm{Tl} \mathrm{ppb}$ & $\mathrm{Uppb}$ \\
\hline LLD $^{1}$ & & & 2.0 & 20 & 100 & 10 & 10 \\
\hline \multicolumn{8}{|l|}{ Guam } \\
\hline GS-4L & Umatac & Facpi Fm & 612 & 33 & 11000 & $<10$ & 115 \\
\hline GS-4LD & duplicate & & 633 & $<20$ & 11500 & $<10$ & 120 \\
\hline GS-8L & Merizo & Facpi Fm joint & 467 & 40 & 13700 & $<10$ & 29 \\
\hline GS-10L & Inarajan & Bolanos Fm & 778 & 35 & 13300 & $<10$ & 52 \\
\hline GS-13L & Umatac & Facpi Fm & 353 & 30 & 14400 & $<10$ & 26 \\
\hline GS-18L & Northern Guam & limestone & 6.0 & $<20$ & 1340 & $<10$ & 325 \\
\hline GS-22L & Central Guam & Alutom Fm & 326 & $<20$ & 4270 & $<10$ & 13 \\
\hline GS-24L & Sella Bay & Facpi Fm & 786 & 16 & 26300 & $<10$ & 60 \\
\hline \multicolumn{8}{|l|}{ Colorado } \\
\hline CS-6L & Lake City & mineralize tuff breccia & 744 & $<20$ & 6420 & $<10$ & 1590 \\
\hline CS-12L & Grand Mesa & basalt & 515 & $<20$ & 5420 & $<10$ & 72 \\
\hline CS-18L & So. Table Mtn. & basalt & 296 & $<20$ & 22800 & $<10$ & 227 \\
\hline
\end{tabular}

${ }^{1}$ lower limit of determination 
Table 9. Chemical analyses of simulated lung fluid dissolution of soils from Guam and Colorado.

\begin{tabular}{lccccc}
\hline Field No & Location & Rock type of formation & V ppb & Y ppb & Zr ppb \\
\hline & & & & & \\
LLD $^{1}$ & & & 20 & 2 & 10 \\
Guam & & & & \\
GS-4L & Umatac & Facpi Fm & 450 & 20 & 183 \\
GS-4LD & duplicate & & 477 & 19 & 211 \\
GS-8L & Merizo & Facpi Fm joint & 784 & $<2$ & 17 \\
GS-10L & Inarajan & Bolanos Fm & 964 & $<2$ & 18 \\
GS-13L & Umatac & Facpi Fm & 558 & $<2$ & 11 \\
GS-18L & Northern Guam & limestone & 112 & $<2$ & 77 \\
GS-22L & Central Guam & Alutom Fm & $<20$ & 18 & $<10$ \\
GS-24L & Sella Bay & Facpi Fm & 92 & 6 & 23 \\
& & & & & \\
Colorado & & & & 16 & 13 \\
CS-6L & Lake City & mineralize tuff breccia & 36 & 16 \\
CS-12L & Grand Mesa & basalt & 161 & $<2$ & 27 \\
CS-18L & So. Table Mtn. & basalt & 427 & 4 & 20 \\
\hline
\end{tabular}

${ }^{1}$ lower limit of determination 